\title{
Microstructure and Property Evolution in Advanced Cladding and Duct Materials Under Long-Term and Elevated Temperature Irradiation: Modeling and Experimental Investigation
}

\section{Reactor Concepts RD\&D \\ Dr. Brian Wirth University of Tennessee at Knoxville}

In collaboration with:

Pennsylvania State University University of South Carolina University of Wisconsin-Madison

Sue Lesica, Federal POC Richard Wright, Technical POC

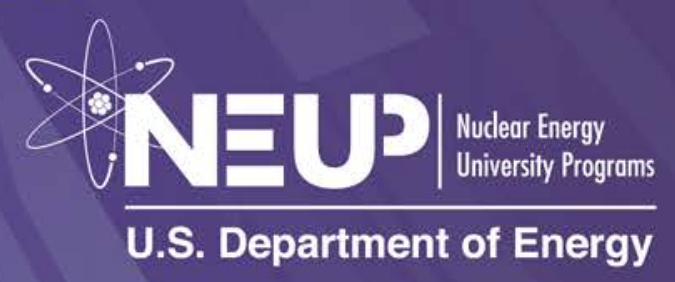


FINAL REPORT FOR

U.S. DEPARTMENT OF ENERGY NUCLEAR ENERGY UNIVERSITY PROGRAMS

Project 10-392

Project Title: Microstructure and Property Evolution in Advanced Cladding and Duct Materials Under Long-Term and Elevated Temperature Irradiation: Modeling and Experimental investigation

Technical Work Scope: G4L-1

Papers and Publications:

D. Kaoumi, J. Adamson, M. Kirk, "Self-Ordered Defect Structures in F/M Steels Under Ion Irradiation”, Journal of Nuclear Materials, under revision (JNM-D-13-00879)

D. Kaoumi, J. Adamson, M. Kirk, "Microstructure Evolution of Two Model Ferritic/Martensitic Steels under In-situ Ion Irradiation at low doses (0-2dpa)", Journal of Nuclear Materials, accepted (JNM-D-12-00671).

D. Xu, X. Hu and B.D. Wirth, “A Phase-Cut Method for Multi-Species Kinetics: Sample Application to Nanoscale Defect Cluster Evolution in BCC Iron Following Helium Ion Implantation", Applied Physics Letters 102 (2013) 011904.

K.G. Field, L. Barnard, C.M. Parish, J.T. Busby, D. Morgan, and T.R. Allen, "Dependence on grain boundary structure of radiation induced segregation in a 9 wt $\% \mathrm{Cr}$ model ferritic/martensitic steel”, Journal of Nuclear Materials 435 (2013) 172-180.

A.P. Selby, D. Xu, N. Juslin, N.A. Capps, and B.D. Wirth, "Primary Defect Production by HighEnergy Displacement Cascades in Molybdenum", Journal of Nuclear Materials 437 (2013) 1923.

D. Xu, B.D. Wirth, M. Li and M.A. Kirk, "Combining in situ transmission electron microscopy irradiation experiments with cluster dynamics modeling to study nanoscale defect agglomeration in structural metals", Acta Materialia 60 (2012) 4286-4302.

D. Xu, B.D. Wirth, M. Li, and M.A. Kirk, "Recent Work Towards Understanding Defect Evolution in Thin Molybdenum Foils Through In-Situ Ion Irradiation Under TEM and Coordinated Cluster Dynamics Modeling”, Current Opinion in Solid State Materials Science 16 (2012) 109-114.

D. Xu, B.D. Wirth, M. Li, and M. A. Kirk, "Defect Microstructural Evolution in Ion Irradiated Metallic Nanofoils: Kinetic Monte Carlo Simulation versus Cluster Dynamics Modeling and InSitu TEM Experiments", Applied Physics Letters 101 (2012) 101905.

Cem Topbasi, Arthur T. Motta, Mark A. Kirk, "In situ study of heavy ion induced radiation damage in NF616 (P92) alloy" Journal of Nuclear Materials 425 (2012) 48-53. 
L. Barnard, G.R. Odette, I. Szlufarska, and D. Morgan, "An ab initio study of Ti-Y-O nanocluster energetics in nanostructured ferritic alloys", Acta Materialia 60 (2012) 935-947.

\section{Invited \& Contributed Oral Presentations:}

B.D. Wirth, A. Kohnert, A. Selby, and D. Xu, "Multiscale Modeling of Defect Cluster Evolution in Irradiated Structural Materials: Focus on thin-film, in-situ IVEM irradiation conditions", TMS 2013 Symposium on Microstructural Processes in Irradiated Materials, San Antonio, TX, 4 March 2013, (invited).

D. Morgan, T. Mayeshiba, L. Barnard, K. Vortler, A. Bengston, and H.O. Nam, "High Throughput Diffusion Modeling for Materials Data and Discovery", The Minerals Metals and Materials Society (TMS), San Antonio, TX, USA, Mar 2013, (invited).

B.D. Wirth, D. Xu, A. Selby, P. Baldo, M. Kirk, and M.-M. Li, "Multiscale Modeling of Radiation Damage and Defect Accumulation Kinetics during Thin-Film Irradiation of Molybdenum: Comparison to In-Situ TEM Observation”, Electron Microscopy Center Review, Argonne National Laboratory, 18 July 2012, (invited).

Cem Topbasi, Arthur T. Motta, Mark A. Kirk, "Development of Radiation Damage in Advanced Steels Using in situ Ion Irradiation" Materials Research Society Fall Meeting; November 2012, Boston, MA, USA .

Cem Topbasi, Arthur T. Motta, Mark A. Kirk, "In situ TEM study of heavy ion induced microstructural evolution in NF616 (P92) alloy", NuMat 2012: The Nuclear Materials Conference; October 2012, Osaka, Japan.

Cem Topbasi, Arthur T. Motta, Mark A. Kirk, "In-Situ Transmission Electron Microscopy Investigation of Microstructural Evolution in complex Ferritic-Martensitic Steels under Ion Irradiation", The Second Workshop on the Use of In Situ TEM/ Ion Accelerator Techniques in the study of Radiation Damage in Solids; June 2011, Albuquerque, New Mexico.

Cem Topbasi, Arthur T. Motta, Mark A. Kirk, "In-Situ Investigation of Microstructure Evolution in NF616 and HCM12A Alloys under Heavy Ion Irradiation", TMS Annual Meeting \& Exhibition; February 2011, San Diego, California, USA.

Cem Topbasi, Arthur T. Motta, Mark A. Kirk, "In-Situ Study of Radiation Damage in Advanced Ferritic-Martensitic Alloys for Gen IV Reactors", The Nuclear Materials Conference; October 2010, Karlsruhe, Germany.

D. Kaoumi, J. Admason, C. Dunn, M. Kirk, "Self-Ordered Defect Structures in Two Model F/M Steels Under In-situ Ion Irradiation", The Nuclear Materials Conference NuMat2012, Osaka, Japan, Oct 2012. 
L. Barnard, G.R. Odette, I. Szlufarska, and D. Morgan, "Ti-Y-O nanocluster energetics in nanostructured ferritic alloys", American Nuclear Society 2012 Winter Meeting, Nov 2012, (invited).

D. Morgan, L. Barnard, K.G. Field, T.R. Allen, I. Szlufarska, and K. Vortler, "First Principles Modeling of Radiation Inducced Segregation in FCC Ni-Cr and FCC Fe-Cr Alloys", Materials Research Society annual meeting 2012, Boston, MA, Nov 2012, (invited).

D. Kaoumi, J. Adamson, B. Wirth, A. Kohnert, M. Kirk, "In-situ Ion Irradiation of Advanced F/M steels for Nuclear Applications", Symposium on Material Challenges in Current and Future Nuclear Technologies, Materials Research Society annual meeting 2011, Boston, MA, Nov 2011, (invited).

J.K. Goddard*, D. Kaoumi, M. Kirk, "Characterization of the dispersion in two ODS steels 18Cr-ODS and 9Cr-ODS", International Conference on Fusion Reactor Materials, ICFRM-15, Charleston, South Carolina, October 16-22, 2011.

J. Adamson*, D. Kaoumi, M. Kirk, "Study of Microstructure Evolution caused by Ion irradiation in Model Ferritic/Martensitic Steels", International Conference on Fusion Reactor Materials, ICFRM-15, Charleston, South Carolina, October 16-22, 2011.

D. Kaoumi, J. Adamson, M. Kirk, T. Faney, B. Wirth, "Microstructure Evolution of Two Model F/M Steels Ion-Irradiated In-Situ in a TEM: Effect of temperature", Symposium on Microstructural Processes in Irradiated Materials at the 2011 TMS Annual Meeting and Exhibition, San Diego, CA, Feb 27 - March 3, 2011.

D. Kaoumi, J. Adamson, M. Kirk, T. Faney, B. Wirth, "Microstructure Evolution of Two Model F/M Steels Ion-Irradiated In-Situ in a TEM", Nuclear Materials Conference 2010, Karlsruhe, Germany October 4-7 2010.

L. Barnard, I. Szlufarska and D. Morgan, "An ab initio investigation of coherent oxide transition phases in nanostructured ferritic alloys", Materials Research Society annual meeting 2010, Boston, MA, Dec 2010. 


\section{Motivation and Objectives}

The in-service degradation of reactor core materials is related to underlying changes in the irradiated microstructure. During reactor operation, structural components and cladding experience displacement of atoms by collisions with neutrons at temperatures at which the radiation-induced defects are mobile, leading to microstructure evolution under irradiation that can degrade material properties. At the doses and temperatures relevant to fast reactor operation, the microstructure evolves by dislocation loop formation and growth, microchemistry changes due to radiation-induced segregation, radiation-induced precipitation, destabilization of the existing precipitate structure, and in some cases, void formation and growth. These processes do not occur independently; rather, their evolution is highly interlinked. Radiation-induced segregation of $\mathrm{Cr}$ and existing chromium carbide coverage in irradiated alloy T91 track each other closely. The radiation-induced precipitation of Ni-Si precipitates and RIS of Ni and $\mathrm{Si}$ in alloys T91 and HCM12A are likely related. Neither the evolution of these processes nor their coupling is understood under the conditions required for materials performance in fast reactors (temperature range $300-600^{\circ} \mathrm{C}$ and doses beyond $200 \mathrm{dpa}$ ). Further, predictive modeling is not yet possible, as models for microstructure evolution must be developed along with experiments to characterize these key processes and provide tools for extrapolation. To extend the range of operation of nuclear fuel cladding and structural materials in advanced nuclear energy and transmutation systems to that required for the fast reactor, the irradiation-induced evolution of the microstructure, microchemistry, and the associated mechanical properties at relevant temperatures and doses must be understood. Predictive modeling relies on an understanding of the physical processes and also on the development of microstructure and microchemical models to describe their evolution under irradiation.

This project will focus on modeling microstructural and microchemical evolution of irradiated alloys by performing detailed modeling of damage evolution processes coupled with well-designed in situ experiments that can provide validation and benchmarking to the computer codes. The project builds upon joint work at the proposing institutions to understand the effects of radiation on these important materials. Complementary to this effort is proposal 10-172, which will focus on critical experiments to improve understanding of the microstructure processes occurring in bulk specimens using proton, ion, and neutron irradiation, on the same group of alloys.

The objective of this proposal is to further advance understanding of highly coupled, irradiation-driven microstructural and microchemical evolutions in ferritic/martensitic alloys, including ODS variants currently under development, which will lead to improved ability to model structural material performance and lifetime predictions.

\section{Results: Experimental Observations of Irradiation Response Materials:}

Two model F/M steels of nominal composition Fe-9Cr-0.1C(wt\%) and Fe-12Cr-0.1C were fabricated at Ames Laboratory, in Iowa by arc melting of $99.98 \%$ purity $\mathrm{Fe}$, $99.995 \%$ purity $\mathrm{Cr}$, and with $99.9995 \%$ purity graphite. The complete chemical composite can be seen in table 1 below. 
Table 1. Material Compositions in wt.\%

\begin{tabular}{|l|l|l|l|l|l|l|l|}
\hline Alloy & Fe & Cr & C & O & N & P & S \\
\hline Fe-9Cr-0.1C & Bal. & 8.68 & 0.125 & 0.0145 & 0.0023 & $<0.005$ & 0.0012 \\
\hline Fe-12Cr-0.1C & Bal. & 12.46 & 0.114 & 0.0098 & 0.0015 & $<0.005$ & 0.0011 \\
\hline
\end{tabular}

As well, two third generation F-M steels, NF616 (ASTM code: P92) and HCM12A (ASTM code: T122), were studied. The NF616 and HCM12A alloys were produced by a sequence of heat treatments of normalization, air cooling and tempering treatments. NF616 was normalized at $1343 \mathrm{~K}$ for $1 \mathrm{~h}$, air cooled, then tempered at $1043 \mathrm{~K}$ for $2 \mathrm{~h}$ and air cooled. Similarly, HCM12A was normalized at $1323 \mathrm{~K}$ for $1 \mathrm{~h}$, air cooled, then tempered at $1043 \mathrm{~K}$ for $45 \mathrm{~min}$ and air cooled.

Table 2 shows the chemical composition of the alloys measured by LUVAK Inc. using inert gas fusion (for oxygen and nitrogen-ASTME 1019-11), combustion infrared detection (for carbon and sulfur-ASTME 1019-11) and direct current plasma emission spectroscopy (other elements -ASTME 1097-12).

Table 2. Composition of NF616 and HCM12A alloys.

\begin{tabular}{|c|c|c|c|}
\hline Element\Alloy & $\begin{array}{l}\text { NF616 } \\
(w t \%)\end{array}$ & $\begin{array}{c}\text { HCM12A } \\
(w t \%)\end{array}$ & $\begin{array}{c}\text { Precision } \\
( \pm w t \%)\end{array}$ \\
\hline Chromium & 9 & 11.09 & 0.2 \\
\hline Carbon & 0.114 & 0.108 & 0.01 \\
\hline Sulfur & 0.004 & 0.002 & 0.002 \\
\hline Nitrogen & 0.045 & 0.059 & 0.002 \\
\hline Oxygen & 0.006 & 0.007 & 0.0005 \\
\hline Phosphorus & 0.013 & 0.012 & 0.002 \\
\hline Boron & $<0.001$ & $<0.001$ & 0.0005 \\
\hline Silicon & 0.11 & 0.25 & 0.005 \\
\hline Vanadium & 0.2 & 0.2 & 0.02 \\
\hline Niobium & 0.097 & 0.087 & 0.005 \\
\hline Manganese & 0.44 & 0.6 & 0.02 \\
\hline Nickel & 0.19 & 0.37 & 0.005 \\
\hline Molybdenum & 0.46 & 0.33 & 0.02 \\
\hline Copper & 0.03 & 1 & 0.002 \\
\hline Tungsten & 1.72 & 1.68 & 0.03 \\
\hline Aluminum & 0.0033 & 0.0027 & 0.0005 \\
\hline
\end{tabular}

The samples for optical microscopy and SEM were initially polished using 180, 400, 800, 1200grit paper followed by final polishing with diamond paste and colloidal silica suspension. The polished samples were etched using the solution with the composition: $45 \% \mathrm{HCl}, 20 \% \mathrm{HNO}_{3}$ and $35 \% \mathrm{H}_{2} \mathrm{O}$. 
The samples for the TEM were first thinned to sheets of thickness $80-120 \mu \mathrm{m}$ by silicon carbide paper. This was followed by mechanical polishing, and then standard TEM discs (3-mm) were punched from the polished foils. Twin-jet electropolishing was used to electropolish TEM samples in $5 \% \mathrm{HClO}_{4}$ and $95 \% \mathrm{CH}_{3} \mathrm{OH}$ solution cooled down to $233 \mathrm{~K}$ by liquid nitrogen.

Figure 1 shows the optical micrographs revealing different microstructures of the two model alloys: a typical martensitic microstructure of laths for Fe-9Cr-0.1C and a ferritic/martensitic microstructure for Fe-12Cr-0.1C (with large grains of ferrite alternating with martensitic regions), all in accordance with the Schaeffler diagram. An average ferrite grain size of $13.3 \mu \mathrm{m}$ was measured for the $\mathrm{Fe}-12 \mathrm{Cr}-0.1 \mathrm{C}$ alloy using the OM micrographs; (the measurement was done by taking the equivalent radius of circular grains of same area).

Figure 2 shows TEM micrographs of the Fe-9Cr-0.1C alloys with the martensitic lath structure and carbides decorating the lath boundaries and the prior austenite grain boundaries (PAGB). The martensitic laths appear to be very narrow (between $200 \mathrm{~nm}$ and 1 micron). The alloy also showed multiple low angle boundaries (middle micrograph). Figure 3 shows the microstructure of the $\mathrm{Fe}-12 \mathrm{Cr}-0.1 \mathrm{C}$ alloy consisting large ferrite grains with very little to no intra-granular carbides alternating with smaller martensitic regions; carbides are observed at the grain boundaries, especially the Ferritic / Martensitic boundaries (middle picture).

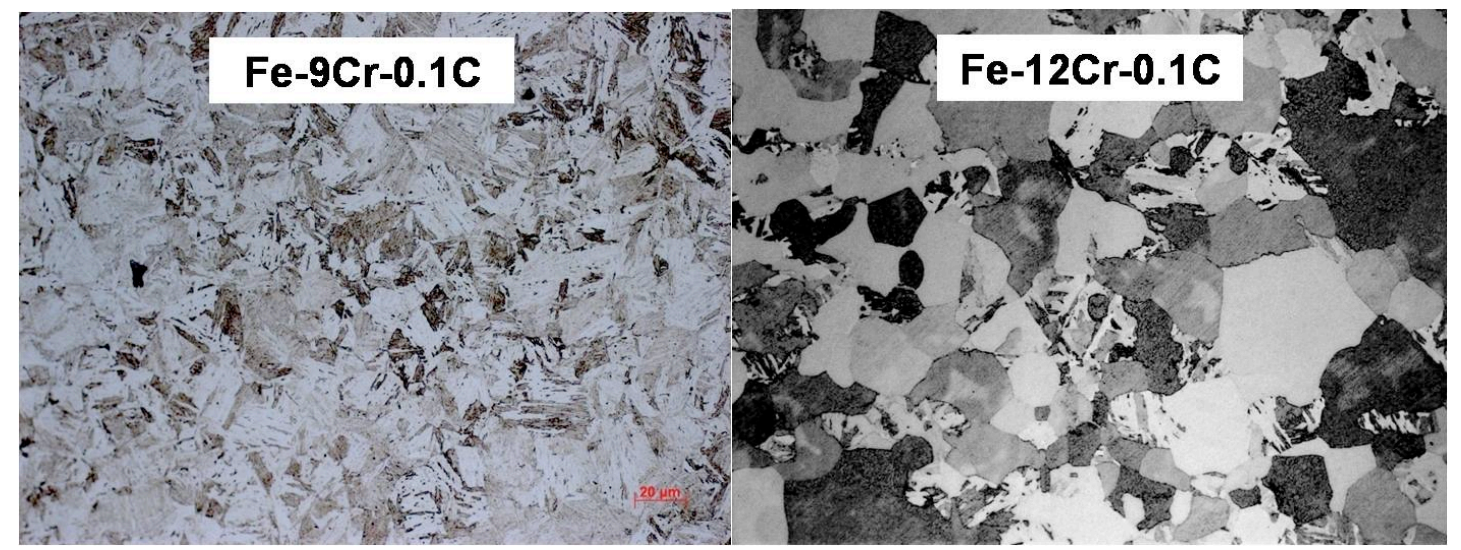

Figure 1. Optical micrographs of model alloys Fe-9Cr-0.1C and Fe-12Cr-0.1C.

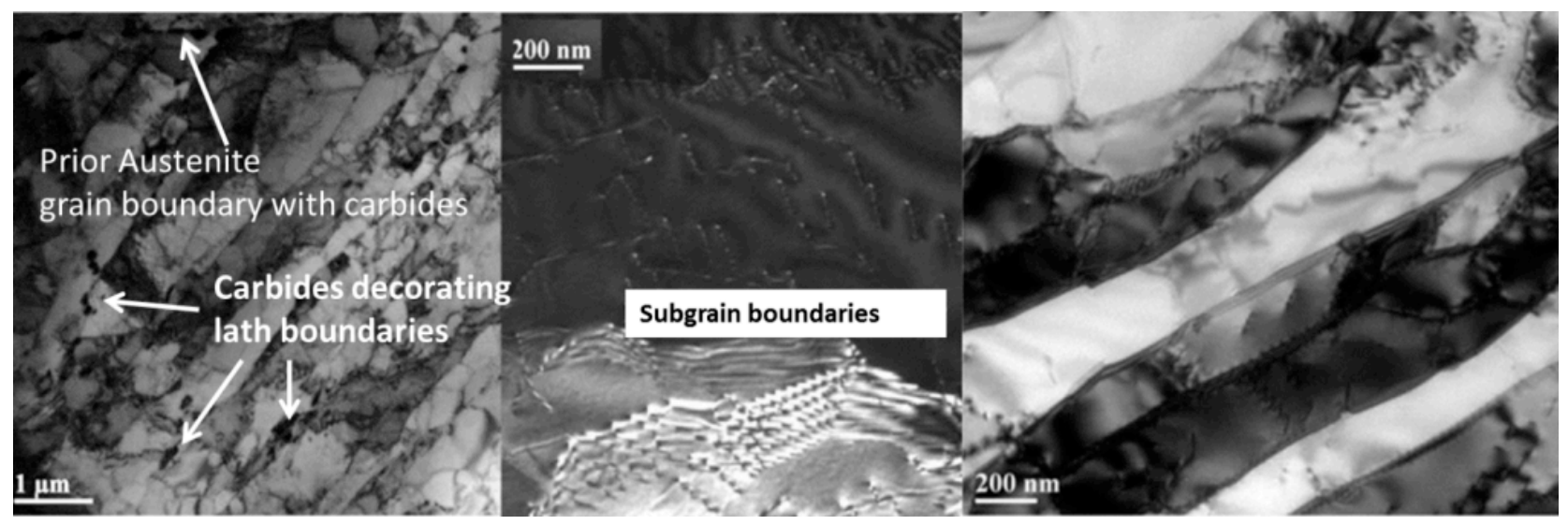


Figure 2. TEM micrographs of model alloys Fe-9Cr-0.1C: (left) martensitic lath structure with carbides decorating lath boundaries and prior austenite grain boundary, (middle) low angle subgrain boundaries; (right) narrow laths.
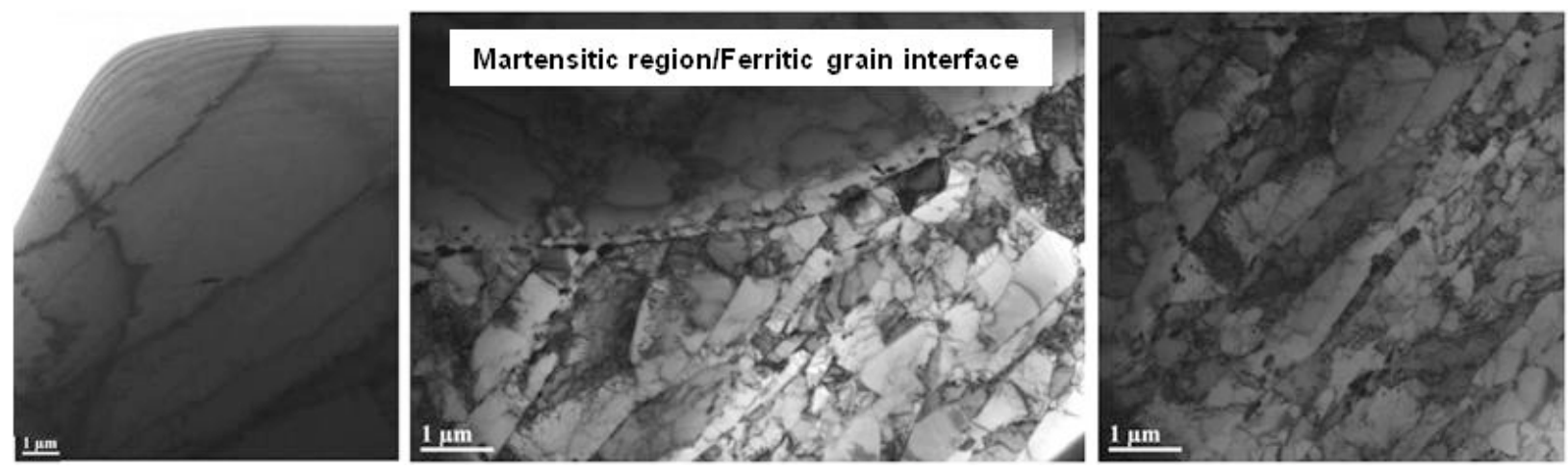

Figure 3. TEM micrographs of model alloys Fe-12Cr-0.1C: (left) ferrite grain; (middle) Ferrite/Martensite interface decorated with carbides, (right) martensitic region.

The microstructure of as-fabricated NF616 and HCM12A was characterized by light optical microscopy, SEM and TEM. Figure 4 shows the optical microscopy images of the typical tempered martensite structure of NF616 and HCM12A at room temperature. Figure 5 shows the secondary electron SEM image of NF616 and HCM12A, which reveals the prioraustenite grains and the lath structure within. Figure 6 shows the bright-field TEM image of the elongated laths that form ferrite grains in both alloys after tempering. Carbide precipitation is observed at lath boundaries and at prior-austenite grain boundaries, with larger precipitates seen at prior-austenite grain boundaries, as shown in Figure 6. Laths exhibit a high dislocation density with significant variability between laths. Occasionally, dislocation networks splits the laths and form sub-cell boundaries, as shown in Figure 6.

The pre-existing dislocation density in HCM12A, although not quantified, was significantly higher than NF616. This can be attributed to the differences in tempering time and the solute atom concentrations. The tempering time was two times less in HCM12A compared to NF616 (45 minutes in HCM12A and 90 minutes in NF616). During tempering of F-M alloys, recovery and precipitation processes occur. Limited recovery and precipitation processes that occurred over a shorter time period can result in the relatively high dislocation and solute atom density in the matrix of HCM12A. 


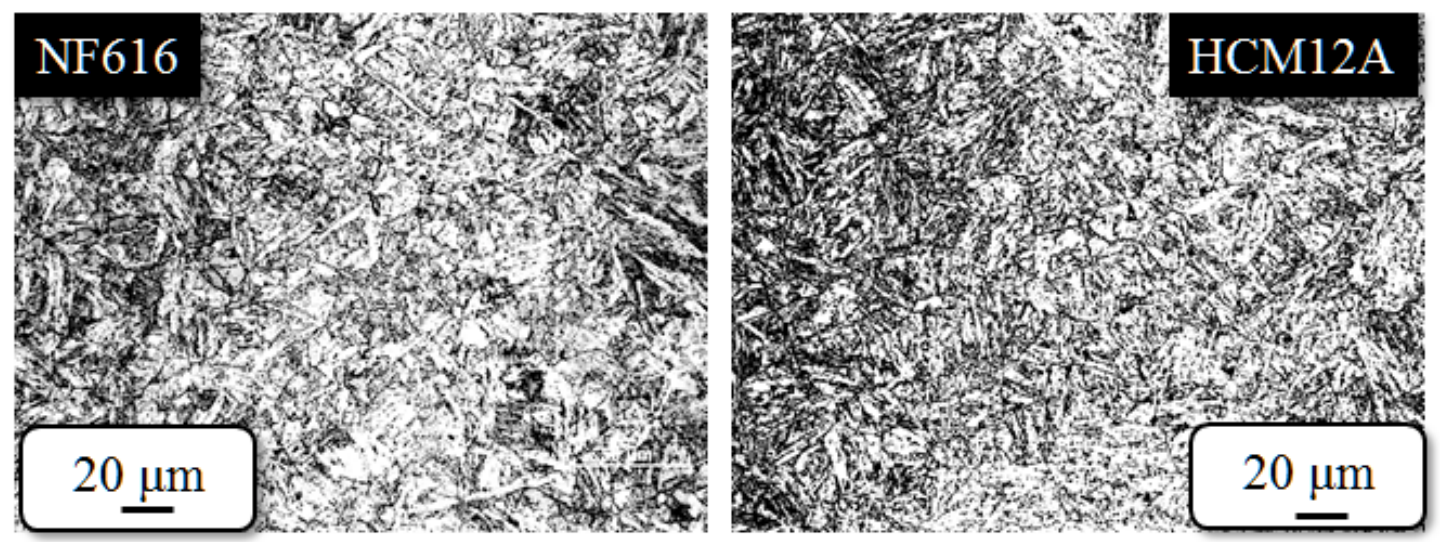

Figure 4. Optical microscopy images of NF616 and HCM12A showing the tempered martensite microstructure.
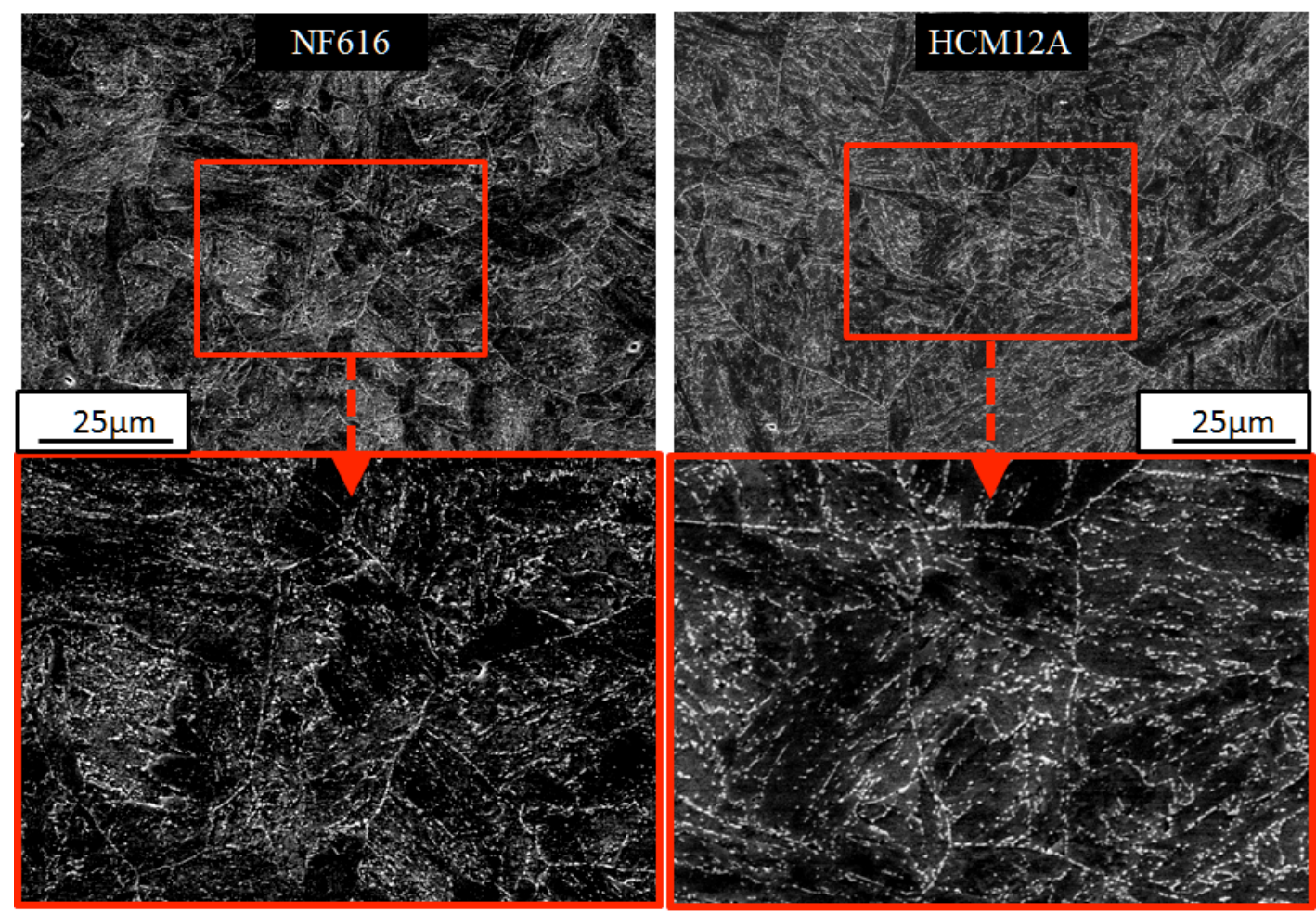

Figure 5. SEM images of prior-austenite grains and the lath structure observed in NF616 and HCM12A. 

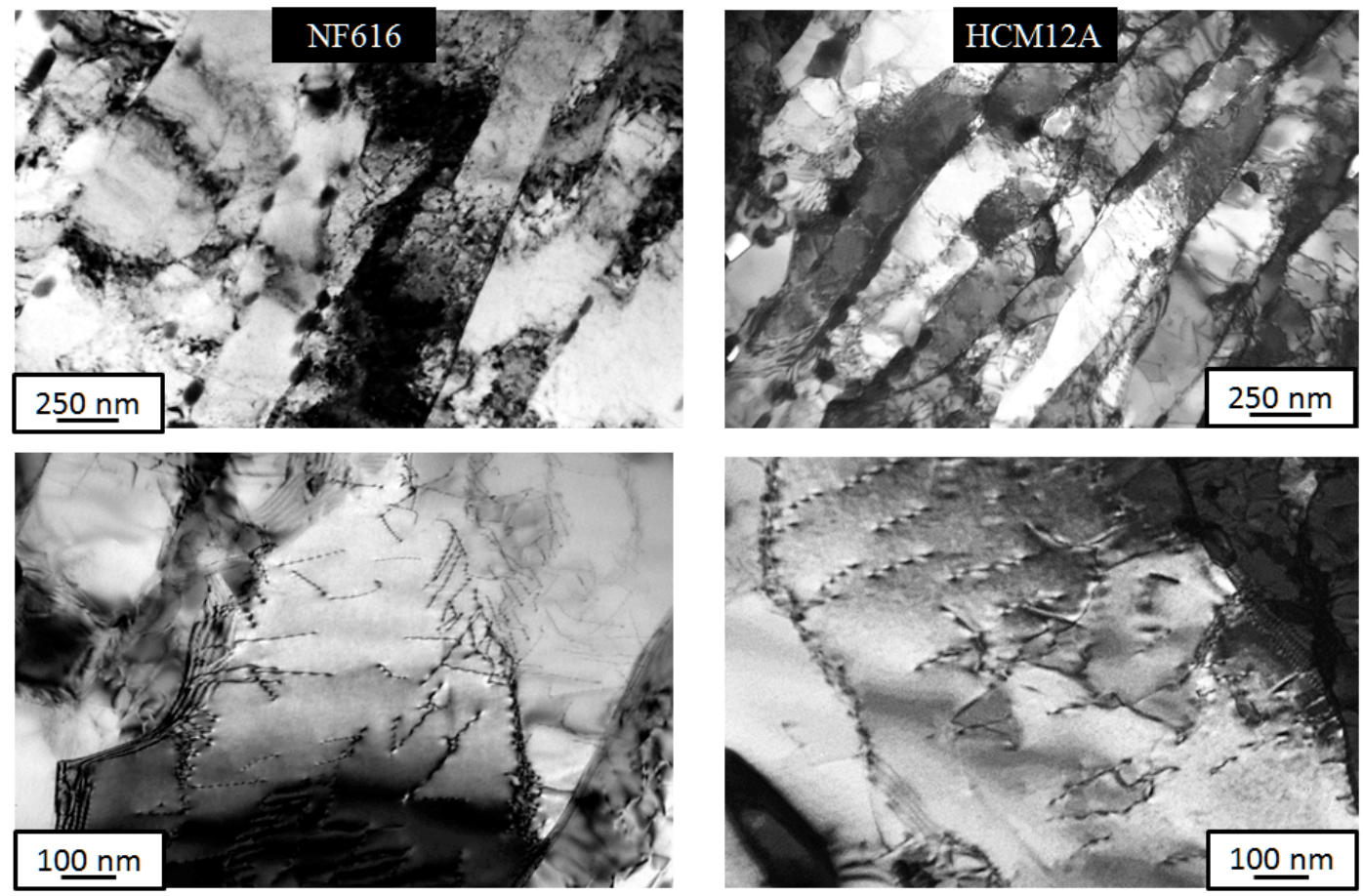

Figure 6. Bright field TEM images of elongated laths (top) and the dislocation structure in weakly recovered laths in NF616 and HCM12A (bottom).

Irradiation Conditions:

In situ heavy ion irradiations of NF616 and HCM12A alloys were performed using the Intermediate Voltage Electron Microscope (IVEM) at Argonne National Laboratory. IVEMTandem is a $300-\mathrm{kV}$ Hitachi H-9000NAR interfaced with ion accelerators where in situ ion beam studies involving ion damage can be conducted. Picture of the IVEM and a schematic illustration of the specimen area inside the microscope are shown in Figure. The angle between the ion beam and the electron beam axis is $30^{\circ}$, allowing continuous observation of microstructure evolution during irradiation. The high angle of incidence is very beneficial in irradiations of F-M alloys since tilting these samples more than $20-30^{\circ}$ is not achievable most of the time due to their ferromagnetic nature. Heating/cooling specimen holder allows in situ experiments in the temperature range of $20-1200 \mathrm{~K}$. The sample temperature is measured using a thermocouple attached to the specimen cup. 


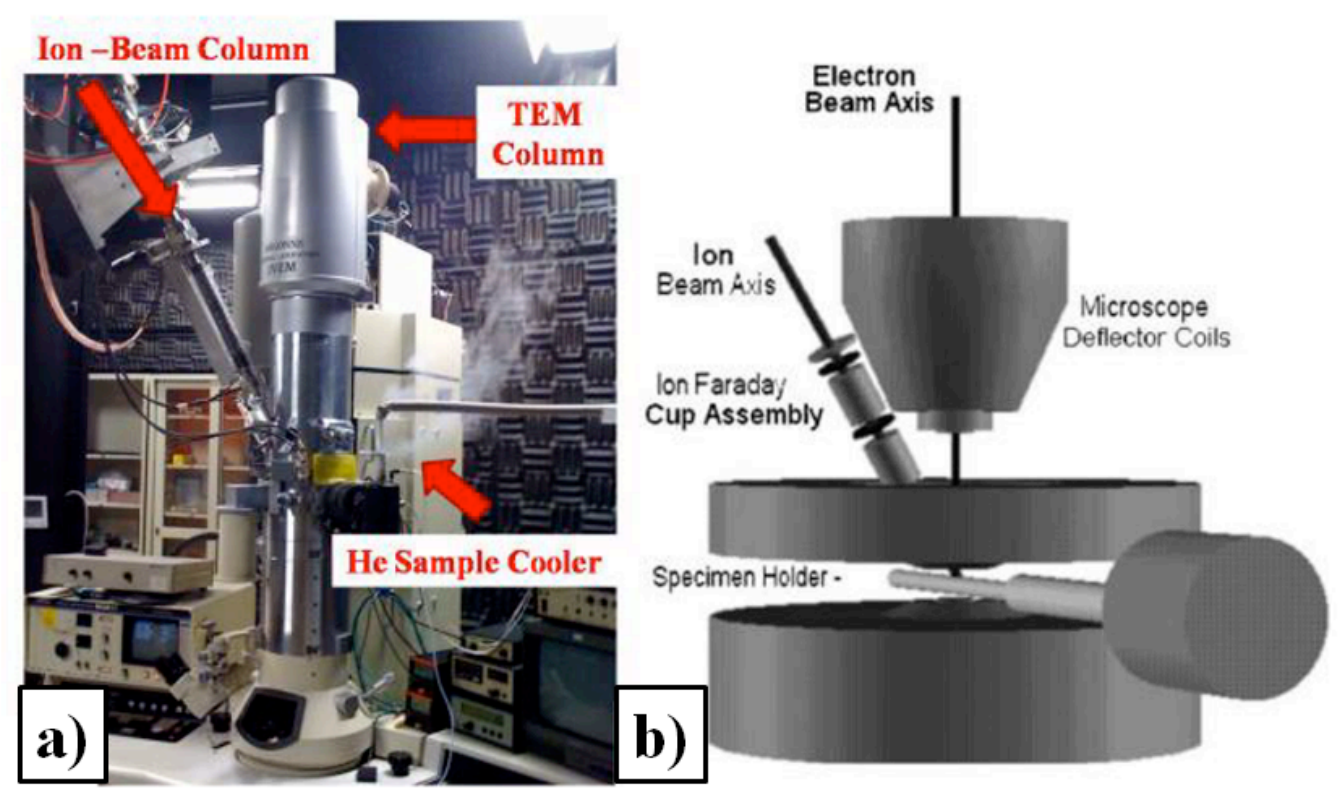

Figure 7. (a) Intermediate Voltage Electron Microscope (IVEM) at Argonne National Laboratory and (b) the illustration of the sample area in the microscope [1].

A series of in situ ion irradiations of electron-thin NF616 and HCM12A alloys were conducted with $1 \mathrm{MeV} \mathrm{Kr}$ ions under a typical flux of $5 \times 10^{11}$ ions. $\mathrm{cm}^{-2} \cdot \mathrm{s}^{-1}$. The microscope was operated at $200 \mathrm{keV}$ thus no displacement damage in Fe is expected from the electron beam.

The energy and ion-type were chosen such that a high displacement damage rate is obtained and implantation of $\mathrm{Kr}$ in the sample is minimized. Furthermore, using a noble gas reduces the possibility of chemical reaction between implanted ions and the atoms in the sample.

The doses in dpa were calculated using the SRIM code, using displacement energy of $40 \mathrm{eV}$ for $\mathrm{Fe}$ and $\mathrm{Cr}$ and $28 \mathrm{eV}$ for $\mathrm{C}$. The damage production cross-section for the $1 \mathrm{MeV} \mathrm{Kr}$ irradiation of NF616 is shown in Figure 1-5. Approximately 95\% of the incident $\mathrm{Kr}$ ions go through the sample, as estimated using SRIM. SRIM calculation performed for $10^{4} \mathrm{Kr}$ ions resulted in the dose rate of $1.44 \times 10^{-3} \mathrm{dpa} / \mathrm{s}$ at an ion flux of $5 \times 10^{11}$ ions. $\mathrm{cm}^{-2} \cdot \mathrm{s}^{-1}$. The results obtained for HCM12A were identical, therefore not given here.

The irradiation induced microstructure evolution of the alloys was observed under particular diffraction conditions. NF616 and HCM12A alloys have $\{111\}$ texture so that generally grains oriented close to zone axis [111] were available for examination. For each irradiation, we set up s-positive 2-beam conditions to image nanometer-sized defects. 110-type g-vectors were consistently used to obtain dark and bright field images to enable direct comparison between images captured at different temperatures and doses.

In the course of the irradiation, specific areas were identified and followed throughout the experiment and irradiation was paused frequently to perform microscopy. Two-beam TEM images were recorded at regular dose intervals. In addition to taking systematic micrographs and diffraction patterns, in situ video recording of microstructural evolution of NF616 and HCM12A was performed using a video-rate camera coupled to the TEM. The high quality videos with time 
resolution of 34 or $67 \mathrm{~ms}$ were used to investigate the dynamic processes (defect creation, elimination and jump characteristics) that occur under irradiation.

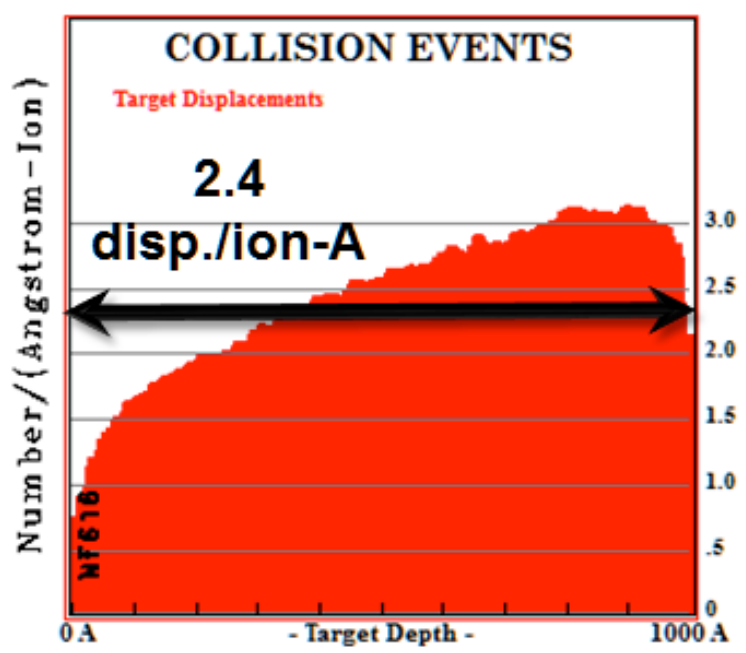

Figure 8. Damage production cross-section for the $1 \mathrm{MeV} \mathrm{Kr}$ irradiation of NF616 calculated by using SRIM. The number of target displacements per ion per angstrom is plotted across the thickness of the sample.

As well, for the model ferritic/martensitic alloys listed in Table 1, experiments were conducted at the ANL IVEM. The model alloy steel was then irradiated with $1 \mathrm{MeV} \mathrm{Kr}$ ions with a count rate of $150 \mathrm{cts} / \mathrm{sec}$, (equivalent to $9.4 \times 10^{11} \mathrm{ion} / \mathrm{cm}^{2} \mathrm{sec}$ ) at $25^{\circ} \mathrm{C}, 200^{\circ} \mathrm{C}$, and $300^{\circ} \mathrm{C}$, up to doses of 10 dpa in-situ in the IVEM. The analysis of the damage structure was followed closely by imaging using several different reflection conditions. Bright-Field and Dark-Field micrographs were systematically taken at the following fluence points: $2.5 \times 10^{13}, 5 \times 1013,1 \times 10^{14}, 2 \times 10^{14}, 3.5 \times 10^{14}$, $5.25 \times 10^{14}, 7 \times 10^{14}, 1 . \times 10^{15}, 1.4 \times 10^{15}, 1.75 \times 10^{15}, 2.8 \times 10^{15}$, and $3.5 \times 10^{15}\left(\mathrm{ions} / \mathrm{cm}^{2}\right)$ corresponding to doses of $\sim 0.075,0.15,0.3,0.6,1.6,2,3,4,5,8$, and $10 \mathrm{dpa}$. The dark-field micrographs were taken in $3 \mathrm{~g}$ (or sometimes higher order) weak beam conditions for optimum defect contrast.

Experimental Results - Model Alloys:

The dark-field micrographs were organized into sequences that showed the evolution of the microstructure caused by the irradiation as illustrated in Figure 9 (top) in the case of Fe-9Cr-0.1C irradiated at $50 \mathrm{~K}$. Overall, the sequences of micrographs revealed a visual increase in the areal number density of visible defect clusters with increasing dose. The density of defect clusters seemed to saturate quickly around 1 to $2 \mathrm{dpa}$. Defect smaller than $\sim 1-2 \mathrm{~nm}$ were below our experimental resolution limit. The nature of the clusters was not determined, but previous studies have shown that dislocation loops with $1 / 2 \mathrm{a}_{0}<111>$ and $<100>$ Burgers vectors form in $\mathrm{Fe}-\mathrm{Cr}$ alloys under irradiation.

At the cryogenic temperatures, the irradiation induced defects did not become visible until higher doses ( $\sim 0.3 \mathrm{dpa}$ at the earliest), compared to the irradiations carried out at higher temperatures (298K and higher) where defects were visible at doses as low as $0.07 \mathrm{dpa}$. This leads to the 
assumption that at cryogenic temperatures $(50 \mathrm{~K}, 180 \mathrm{~K})$ a large density of small defects under the TEM resolution limit are originally formed and become detectable i.e. visible only when higher doses are reached after they have grown. The mechanisms of growth are uncertain but a credible assumption is that, at $50 \mathrm{~K}$ (stage I for Fe-Cr system), the clusters form, not from the direct collapse of individual displacement cascades, but from spatial overlap of cascades i.e. defect cluster form and grow when the damage cascade created by an ion impact overlaps with the remains of a previous cascade, which does not require long range diffusion. The fact that at higher temperatures the defects are visible at much earlier doses may be due to enhanced incascade clustering, and enhanced interaction of defects during cascade overlap at higher temperatures. 

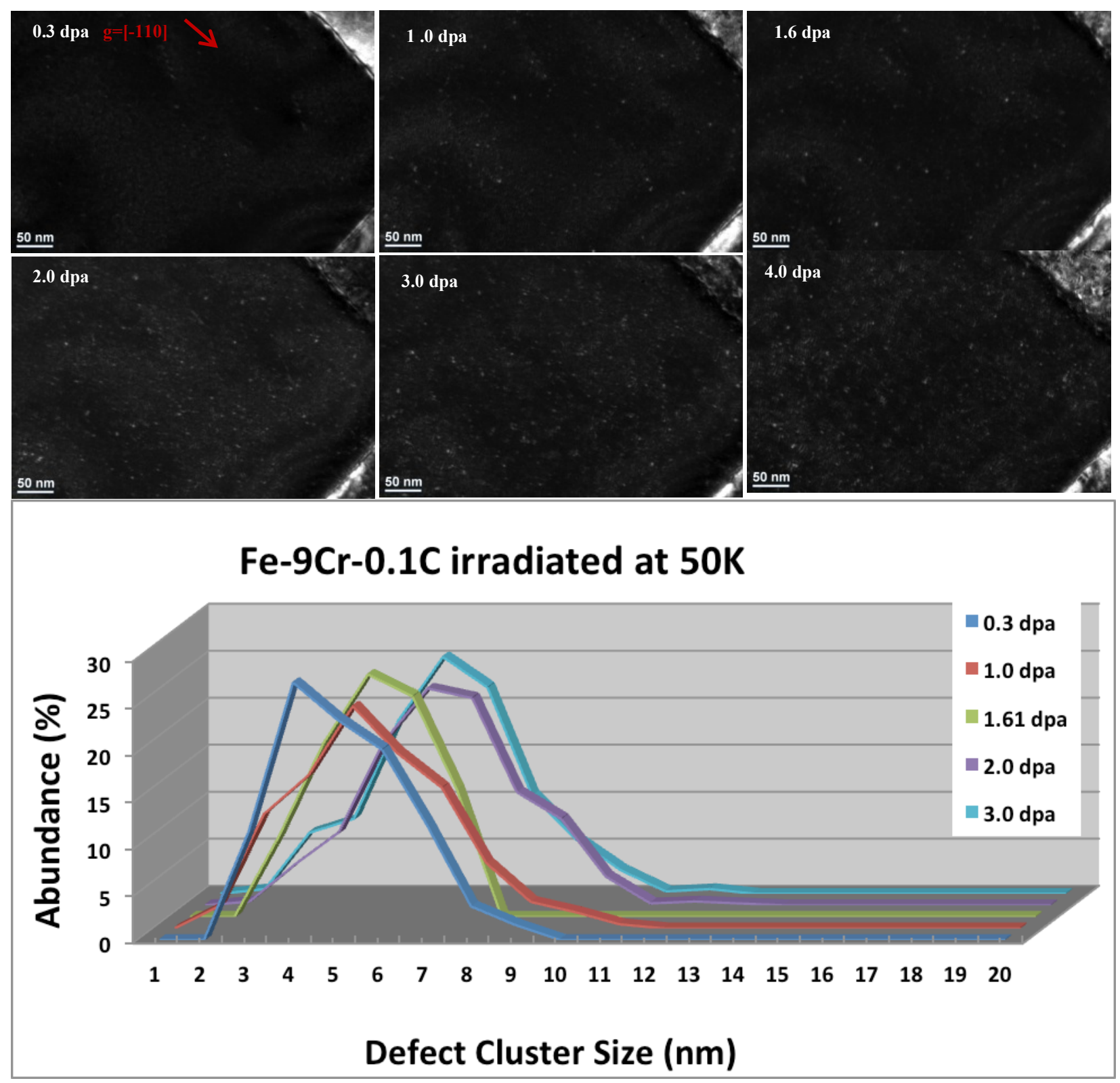

Figure 9. (Top) Dark Field TEM micrographs of model alloy Fe-9Cr-0.1C irradiated at 50K to doses ranging from 0.3 to $4 \mathrm{dpa}$ with $1 \mathrm{MeV} \mathrm{Kr}^{2+}$ ion $(\mathrm{z}=[001] \mathrm{g}=[-110])$; (bottom) Corresponding cluster size distributions.

The defect clusters were counted and measured using Adobe Photoshop software. Of course, the visibility of defects depends on their size and the resolution limit which in turn depends on the microscope and the sample surface quality. In general, the smallest visible white dots were found to be between 1 and $2 \mathrm{nms}$, which can be taken as the resolution limit. It is also important to note that, as predicted by the $\mathrm{g} \bullet \mathrm{b}$ invisibility criterion, even if they are larger than the resolution limit, under the imaging conditions used not all clusters and loops will be visible. In fact, when using a 110-type g vector only half of the $\mathbf{a}_{\mathbf{0}} / 2$ [111] loops and one-third of the $\mathbf{a}_{0}$ [100] loops can be imaged. 
Depending on the quality of the image (surface roughness, and imaging conditions), some defects appeared more clearly than others; therefore, the defects were categorized into one of two bins "certain" or "uncertain" and two density values were calculated; the lower value corresponds to the number of defects which were counted as "certain" and the higher value accounts both for "certain" and "uncertain" defects; this allowed to estimate the error associated with the measurement as plotted in figure 10 .

The defects sizes were measured with the picture zoomed in to its actual size to show the actual pixels. The measurements were then put into a spreadsheet that converted the pixel measurement into $\mathrm{nm}$ using the scale bar from each photo. The defects usually showed a noncircular aspect with a small diameter and a large diameter, which led to the assumption of an elliptic shape for all defects. The size of the defect was then taken to be the diameter of a disk of equivalent area. Defect size distributions were plotted for each dose. For example, the defect size distributions corresponding to the sequence of dark field micrographs of the region followed throughout the irradiation of $\mathrm{Fe}-9 \mathrm{Cr}-0.1 \mathrm{C}$ at $50 \mathrm{~K}$ are plotted in figure 5 (bottom), using a bin size of $1 \mathrm{~nm}$. The distributions typically showed a single nodal bell shape indicating that the detectable defect size follows a normal distribution. The peak of the distribution is seen to shift to larger sizes as the dose is increased which translates into an increase in average size with increasing dose.

Note: When analyzing such defect size distributions, it is important to remember that one limitation of TEM defect measurements stems from the fact that there is a fraction of defects whose size is below the resolution limit of the TEM; this could lead to under-estimate the number of defects and possibly result in overestimating the measured average size of the (detectable) defects, by shifting the size distributions to larger sizes because the smallest defect cluster cannot be measured. Also it is likely that defect size distribution will vary systematically with formation temperature, as the proportion of defects which are too small to be resolved changes with temperature.

Defect areal density vs. dose vs. temperature: The areal defect densities were found by dividing the total number of defects by the actual area of the micrograph being analyzed. The defect densities were plotted as a function of dose and irradiation temperature in Figure 10. Generally, the measured areal defect density increases with dose until it reaches an apparent saturation value, early in dose . It is worth mentioning that these measurements were made without correcting for systematic absences under the $(\mathrm{g} \bullet \mathrm{b}=0)$ invisibility criterion or for loss of glissile loops to the foil surfaces. The curves were normalized to the highest density measured at $50 \mathrm{~K}$.

The effect of temperature is similar for both Fe-9Cr-0.1C and Fe-12Cr-01C systems: overall, the defect density decreases with increasing irradiation temperature. At the lowest temperature, the defects mobility is limited; the clusters therefore remain in their initial position of formation unless they interact with other successive overlapping cascades. This leads to a high concentration in the matrix. On the other hand, when the temperature is increased, there is a higher probability for defects to move and get lost to sinks such as grain-boundaries and free surfaces, or they may contribute to the growth of the larger loops; this all leads to smaller saturation defect densities but larger loops on average as shown below. 


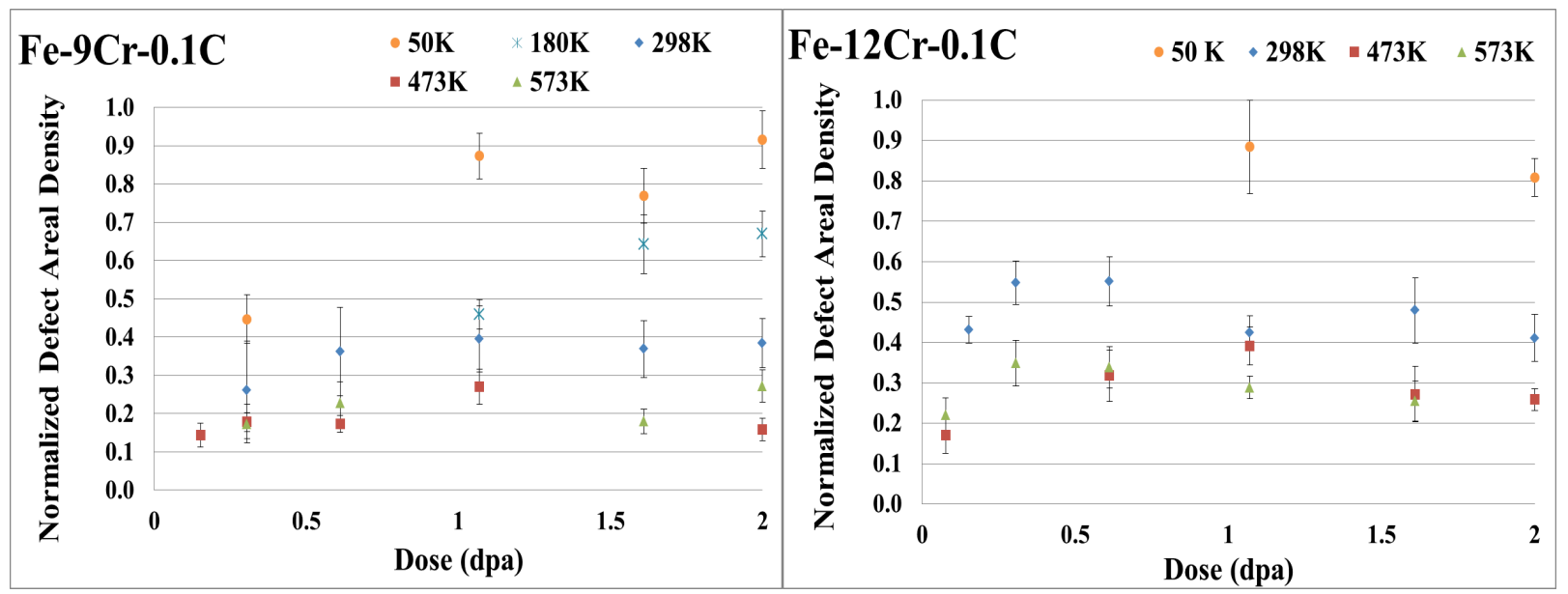

Figure 10. Defect areal density vs. dose vs. irradiation temperature for the 9Cr model alloy and $12 \mathrm{Cr}$ model alloy.

Average loop size vs. dose vs. temperature: The average size of the detected defect clusters was calculated for each dose and plotted vs. dose and temperature in Figure 11. The average defect size increases with increasing dose following an apparent linear trend. As for the effect of temperature, overall, the average loop size increases with increasing temperature (reverse trend of the defect density vs. temperature). The exception to the trend observed in Fe-9Cr-0.1C at $573 \mathrm{~K}$ (where the average size of the loops being smaller than the value at $473 \mathrm{~K}$ ) may be due to the smaller grain size of this alloy (i.e. higher grain-boundary density) compared with $\mathrm{Fe}-12 \mathrm{Cr}$ 0.1C. At these temperatures, the defects have a high probability of reaching a sink (e.g. grain boundaries), reducing their chance to contribute to the growth of other defects.

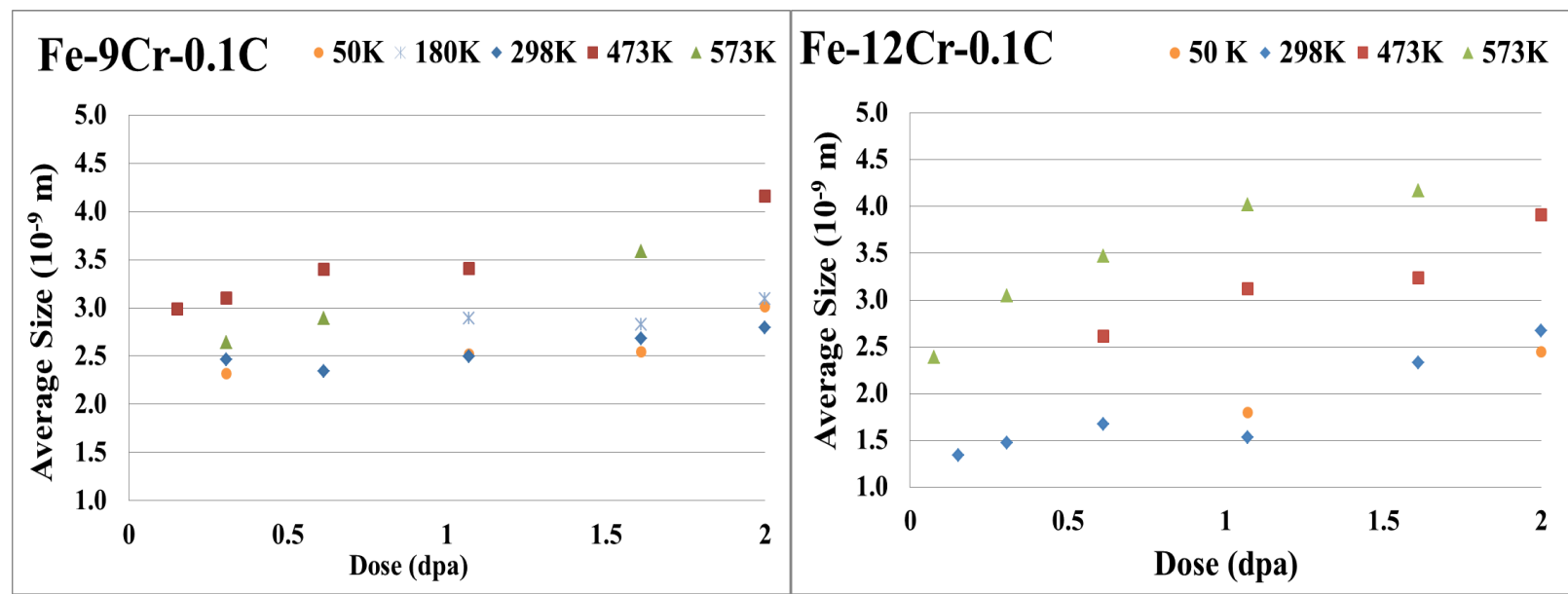

Figure 11. Average visible defect size vs. dose vs. irraditation temperature for the $9 \mathrm{Cr}$ model steel and $12 \mathrm{Cr}$ model steel.

Maximum loop size vs. dose vs. temperature: At each dose, the maximum loop size was found by calculating the average size of the top 5\% largest loops (for statistical representation). Figure 12 shows the maximum defect cluster size as a function of dose and temperature. Trends similar to those of the average loop size are observed i.e. an apparent linear increase of size vs. dose and an 
overall increase of the maximum loop size with increasing temperature except for the $\mathrm{Fe}-9 \mathrm{Cr}-$ $0.1 \mathrm{C}$ at $573 \mathrm{~K}$.

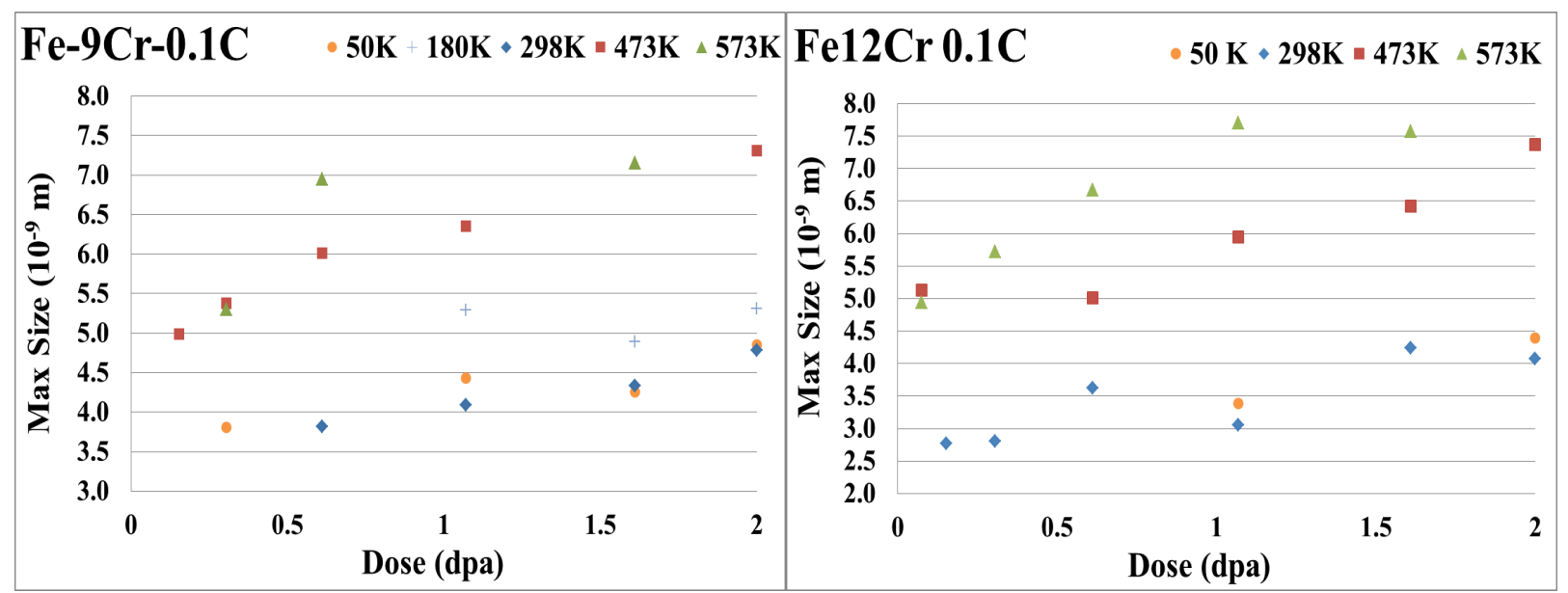

Figure 12. Maximum loop size vs. dose vs. irraditation temperature for the $9 \mathrm{Cr}$ model and $12 \mathrm{Cr}$ model steel.

During the irradiation of the model F/M steel, at the very early stages of irradiation, defect clusters appear to be rather uniformly distributed within grains, and the saturation density is quickly reached between 1.0 and $2.0 \mathrm{dpa}$. However, some ordered alignments of defect clusters are found in some grains as shown in Fig. 13. Ordered arrays of small loops with spacing about $30-50 \mathrm{~nm}$ are observed after irradiation with $\mathrm{Kr}$ ions. Once this aligned structure is created (sometimes as early as $3 \mathrm{dpa}$ ), it is stable up to $10 \mathrm{dpa}$. Such microstructure evolution was also observed in the Fe-9Cr-0.1C steel despite the difference in initial microstructure (Fe-12Cr-0.1C is $\mathrm{F} / \mathrm{M}$ whereas Fe-9Cr-0.1C is fully $\mathrm{M}$ ).

In order to find the possible mechanisms for the "self-organization" or "patterning" of the irradiation induced clusters, the effect of temperature was investigated systematically to assess the role (or absence of role) played by radiation enhanced diffusion for the process to happen. Irradiations were done at $20 \mathrm{~K}, 293 \mathrm{~K}, 573 \mathrm{~K}$. The cluster alignment patterning was observed at $20 \mathrm{~K}$ and at $298 \mathrm{~K}$, but it was not observed at $573 \mathrm{~K}$ and above. Figure 13 shows a dark field micrograph of model alloy Fe-12Cr-0.1C irradiated up to $11.4 \mathrm{dpa}$ at $20 \mathrm{~K}$ (Z=[111]); the selforganized defect alignment was observed in both martensitic and ferritic regions. The directions of alignment correspond to the same equivalent crystallographic $<110>$ directions. 




Figure 13. Defect alignment in Fe-12Cr-0.1C irradiated at $20 \mathrm{~K}$ to $11.4 \mathrm{dpa}$; (left): martensitic region, (right): ferrite grain $(Z=[111])$.

Mechanism and Driving Force: This structure is thought to result from elastic interactions between defect clusters in a thin foil; the loop accumulation and their interactions may form the defect arrays. The fact that such defect alignment was not observed at higher temperatures suggests that the relatively high density of defect clusters (at lower temperatures) and the resultant internal strains may be the main reason for the development of the aligned structure. Crystallographic orientation of defect arrays may be enhanced in order to minimize elastic interaction energy between defect clusters. The stress caused by a high density of loops would be minimized by the regular arrangement of defects clusters, which could be a driving force for the process.

Development of the alignment structure: During the irradiations, such regions subject to loop patterning were followed in-situ and movies were recorded. Figure 14 shows a sequence of still pictures taken from a video recorded in-situ during the $1 \mathrm{MeV} \mathrm{Kr}$ ion-irradiation $\mathrm{Fe}-12 \mathrm{Cr}-0.1 \mathrm{C}$ irradiation at room temperature. The rattling motion (moving down then up) of a cluster (yellow arrow) is observed. The cluster moves down $(\mathrm{a} \rightarrow \mathrm{b})$, then up to the initial position $(\mathrm{c} \rightarrow \mathrm{d})$, then it moves up $(\mathrm{e} \rightarrow \mathrm{f})$, then up again $(\mathrm{g} \rightarrow \mathrm{h})$, and $(\mathrm{i} \rightarrow \mathrm{j})$; then it is close enough to the previously aligned segment and they interact attractively: the cluster now moves from right to left towards the segment $(\mathrm{k} \rightarrow 1 ; \mathrm{m} \rightarrow \mathrm{n})$ and finally the cluster is sucked into the segment $(\mathrm{o} \rightarrow \mathrm{p})$ contributing to its reinforcement and development. The recorded movie shows how this mechanism allows the aligned structure to develop.

Dislocation loop segments seem to extend by addition of clusters moving in their direction. Figure 10 shows a sequence of dark-field still images taken from a video of the $12 \mathrm{Cr}$ model steel irradiated at $20 \mathrm{~K}$ recorded in-situ and evidences growth, change in curvature, and merger of segments. The yellow circle shows the growth and merger of two neighbouring segments; the red circle highlights the gradual change of curvature of two segments enabling their merger. Such processes are responsible for the final configuration of regularly spaced arrays. 




$\mathrm{t}+0.28$

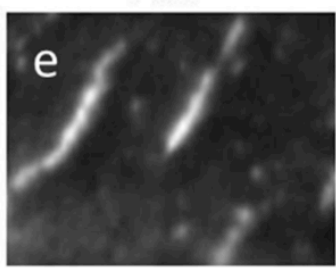

$\mathrm{t}+2.92$

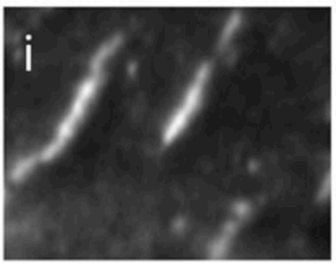

$t+4.96$



$\mathrm{t}+10$

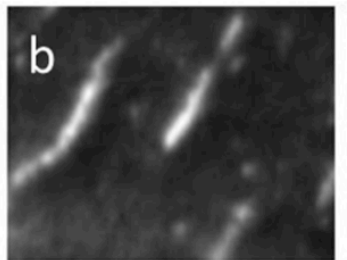

t+0.32

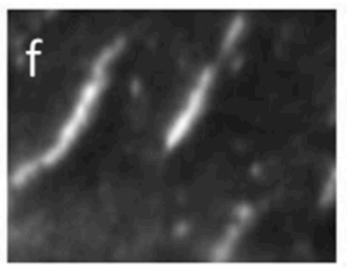

$t+2.96$

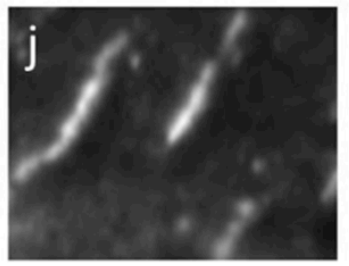

$t+5$

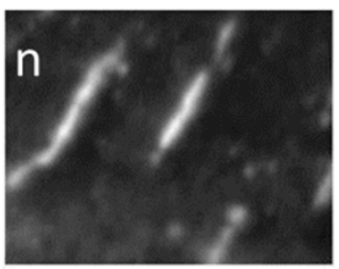

$t+10.04$

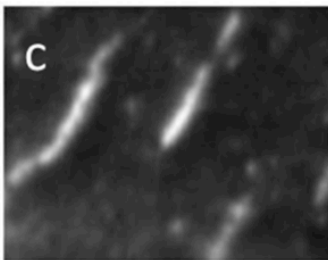

$t+1.56$

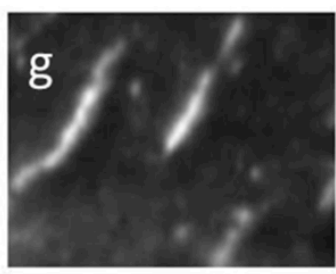

$t+4.36$

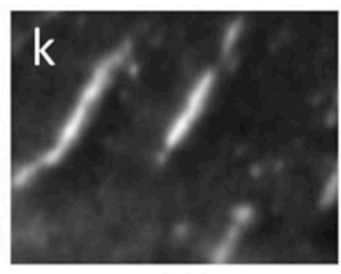

$t+9.96$

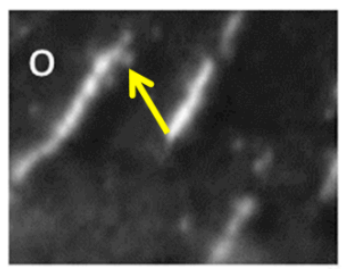

$t+19.84$



$\mathrm{t}+1.6$

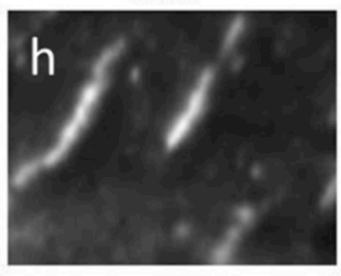

$t+4.4$

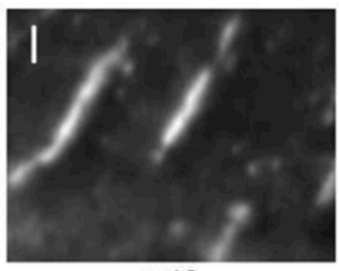

$t+10$

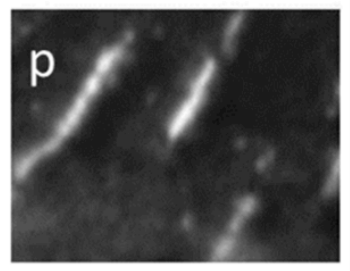

$t+19.88$

Figure 14. Sequence of still pictures from taken from the video of the $1 \mathrm{MeV} \mathrm{Kr}$ ion irradiation of $\mathrm{Fe}-12 \mathrm{Cr}-0.1 \mathrm{C}$ at $25 \mathrm{C}(\mathrm{Z}=[111])$.

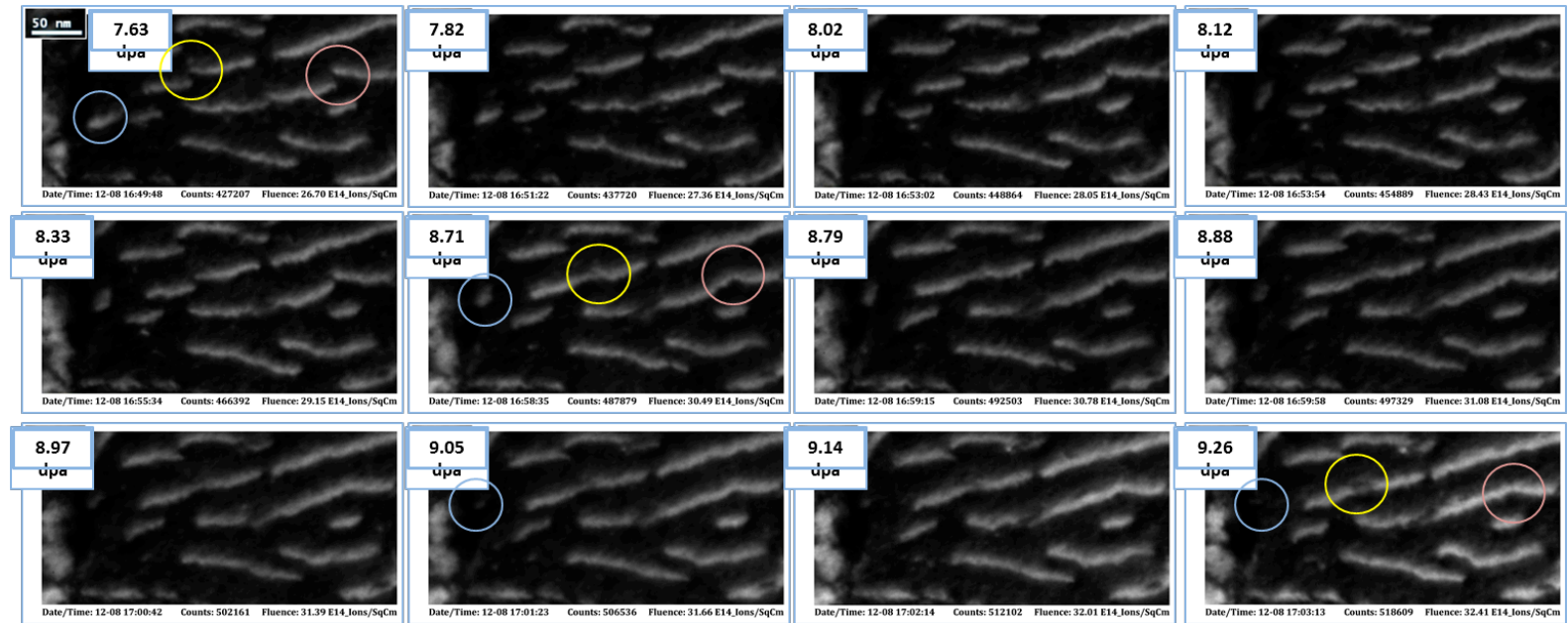

Figure 15. Dislocation loop segments extending by addition of clusters moving in their direction observed at $20 \mathrm{~K}$ in $\mathrm{Fe}-12 \mathrm{Cr}-0.1 \mathrm{C}$ : the merger of two segments and the change of curvature of another segment are circled. 
Summary of irradiation of model ferritic/martensitic alloys:

Low doses (0-2dpa): Kinetics data of microstructure evolution in two model ferritic/martensitic steels (of nominal compositions $\mathrm{Fe}-9 \mathrm{Cr}-0.1 \mathrm{C}$ and $\mathrm{Fe}-12 \mathrm{Cr}-0.1 \mathrm{C}$ ) under $1 \mathrm{MeV} \mathrm{Kr}^{2+}$ ion irradiation was measured in terms of irradiation-induced defect cluster density, average defect size and maximum defect size as a function of dose and temperature (up to $2 \mathrm{dpa}$ ). At a given temperature, the density of defect clusters increases with dose to an apparent saturation value reached early in dose (between 1 and $2 \mathrm{dpa}$ ). The saturation defect density decreases with increasing irradiation temperature. The size of the loops seems to grow linearly with dose at all temperatures; however larger loops are formed at larger temperatures. Given the range of temperatures spanned for these experiments, two distinct aspects of temperature effects were considered to explain the effect of temperature: (i) the nature of the defects able to migrate at the temperature of consideration (as identified in studies of resistivity recovery stages) and (ii) the effect of temperature on the mobility/diffusivity of the defects.

Higher doses (2-10 dpa): Self ordered dislocation loops observed: Also, during the early stages of irradiation of the two F/M steels, defect clusters appear to be rather uniformly distributed within grains, and the saturation density is quickly reached between 1.0 and $2.0 \mathrm{dpa}$. However, self-ordering alignments of defect clusters are found in some grains at doses as low as $3 \mathrm{dpa}$. The regularly ordered arrays of small loops with spacing about 30-50 nm are observed in the two F/M steels along $\langle 110\rangle$ directions. Once the aligned structure is created, it is stable under further irradiation. This structure is thought to result from elastic interactions between defect clusters in the foil. The fact that such defect alignment is not observed at higher temperatures suggests that the relatively high density of defect clusters at lower temperatures and the resultant internal strains may be the main reason for the development of the aligned structure i.e. The stress caused by a high density of loops would be minimized by the regular arrangement of defects clusters. The preferred crystallographic orientation of defect arrays may be driven by the minimization of elastic interaction energy between defect clusters.

Experimental Results - Commercial Alloys:

In this section, the results of the in situ irradiations of NF616 and HCM12A are presented.

The irradiations were performed with $1 \mathrm{MeV} \mathrm{Kr}$ ions between 20 and $773 \mathrm{~K}$ to investigate the microstructure evolution of these alloys in different temperature regimes.

The results include diffraction patterns and TEM images captured at specific doses, as well as the video frames extracted from the in situ irradiation videos. In addition quantitative analysis was performed on TEM images and video frames to determine defect size and density by manually analyzing defect clusters using ImageJ software. Similarly, the jump distance and direction of specific defect clusters were individually determined by conducting frame-by-frame analysis of videos before and after the motion.

The detailed analyses performed on TEM micrographs and video frames lead to the following general results about the microstructure evolution of NF616 and HCM12A alloys.

Low-temperature Region:

The results of in situ irradiations of NF616 and HCM12A between 20 and $573 \mathrm{~K}$ are given in this section. As the irradiation started, there was a period during which no changes to the 
microstructure were visible. After a threshold dose, defects in the form of white dots started to become visible in dark field TEM images under the ion beam.

Figure 16, shows the series of two-beam DF TEM images of the same area in NF616 captured at $10^{-3}, 10^{-2}, 10^{-1}$ and $1 \mathrm{dpa}$ at room temperature. There were no visible defects at $10^{-3}$ and $10^{-2} \mathrm{dpa}$; on the other hand, $2-5 \mathrm{~nm}$ defects were visible as white dots in two-beam DF images at $10^{-1} \mathrm{dpa}$. The dose for the onset of defect accumulation was determined to be $0.06 \mathrm{dpa}$ at $298 \mathrm{~K}$ by frameby-frame analysis of the irradiation video. Approximately 2 ions were incident per $1 \mathrm{~nm}^{2}$ at this dose point; therefore it is evident that spatial overlapping of cascades was involved in the defect creation process.

Figure 17 and 18 show the initial stages of defect accumulation in NF616 and HCM12A between 20 and $573 \mathrm{~K}$. The onset of defect accumulation in both alloys was just below 0.1 dpa between 20 and $573 \mathrm{~K}$, similar to the RT irradiation of NF616 shown in Figure 16.
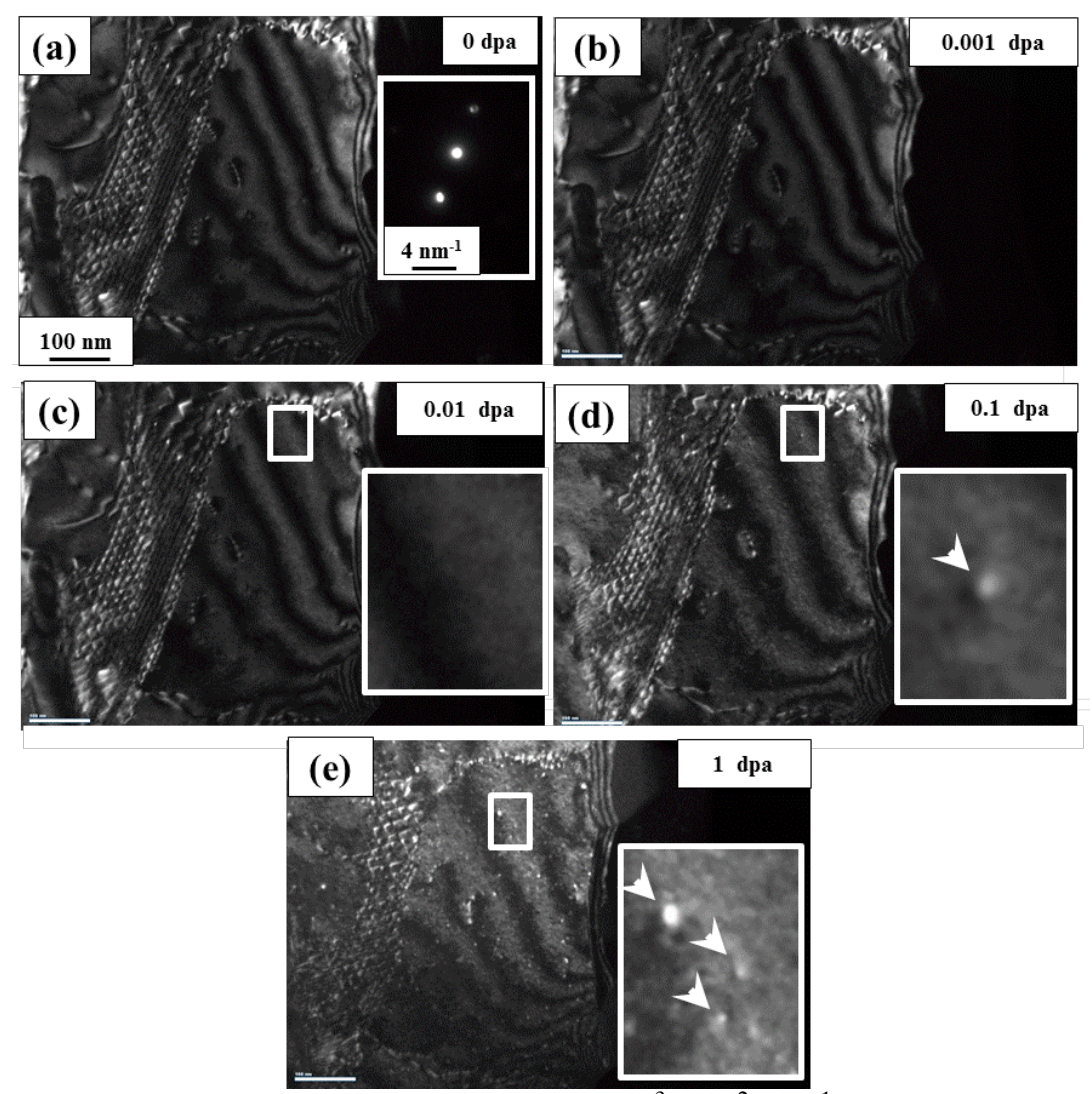

Figure 16. DF TEM images of NF616 irradiated to $10^{-3}, 10^{-2}, 10^{-1}$ and 1 dpa at room temperature with $1 \mathrm{MeV} \mathrm{Kr}$ ions. 

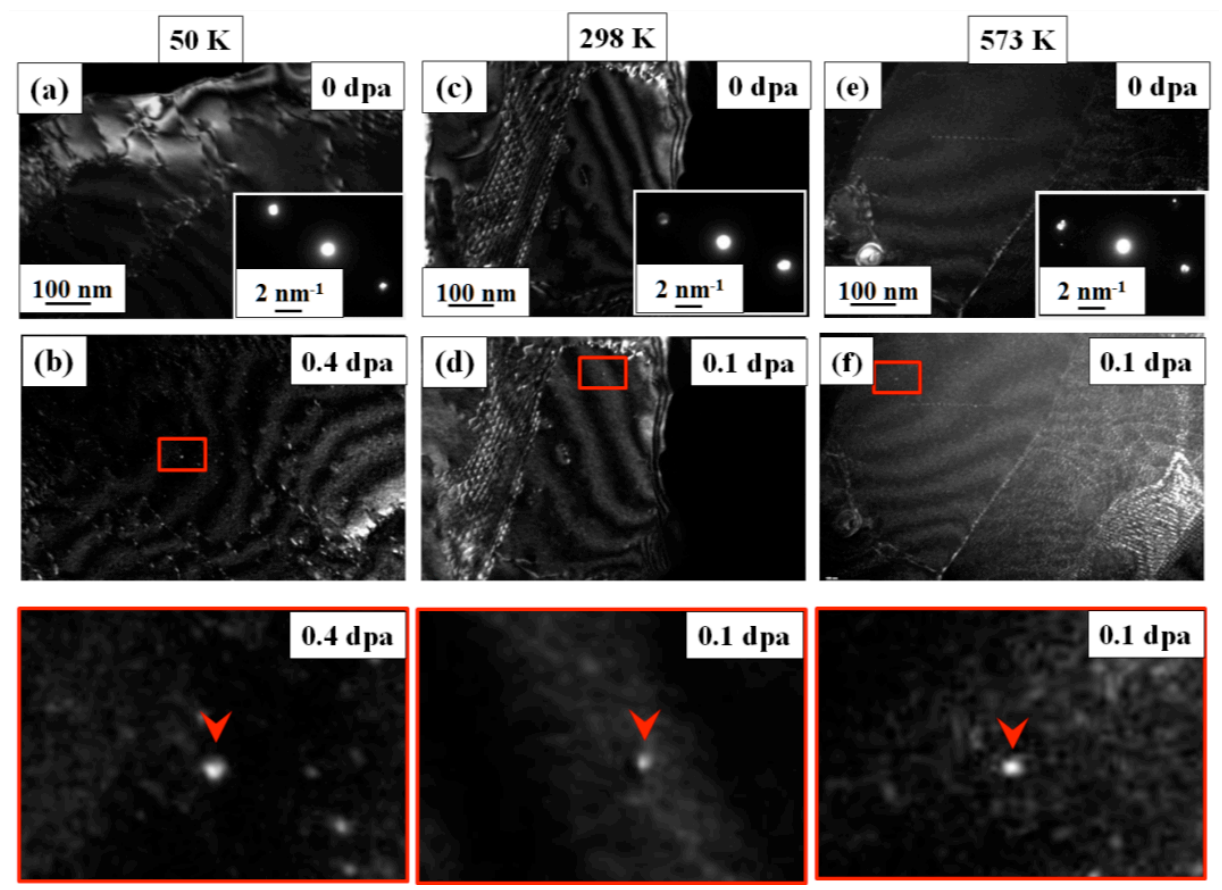

Figure 17. TEM images showing the initial stages of defect accumulation in NF616 at 50, 298 and $573 \mathrm{~K}$. Insets of figures show the two beam condition and the close up view of the defect clusters.
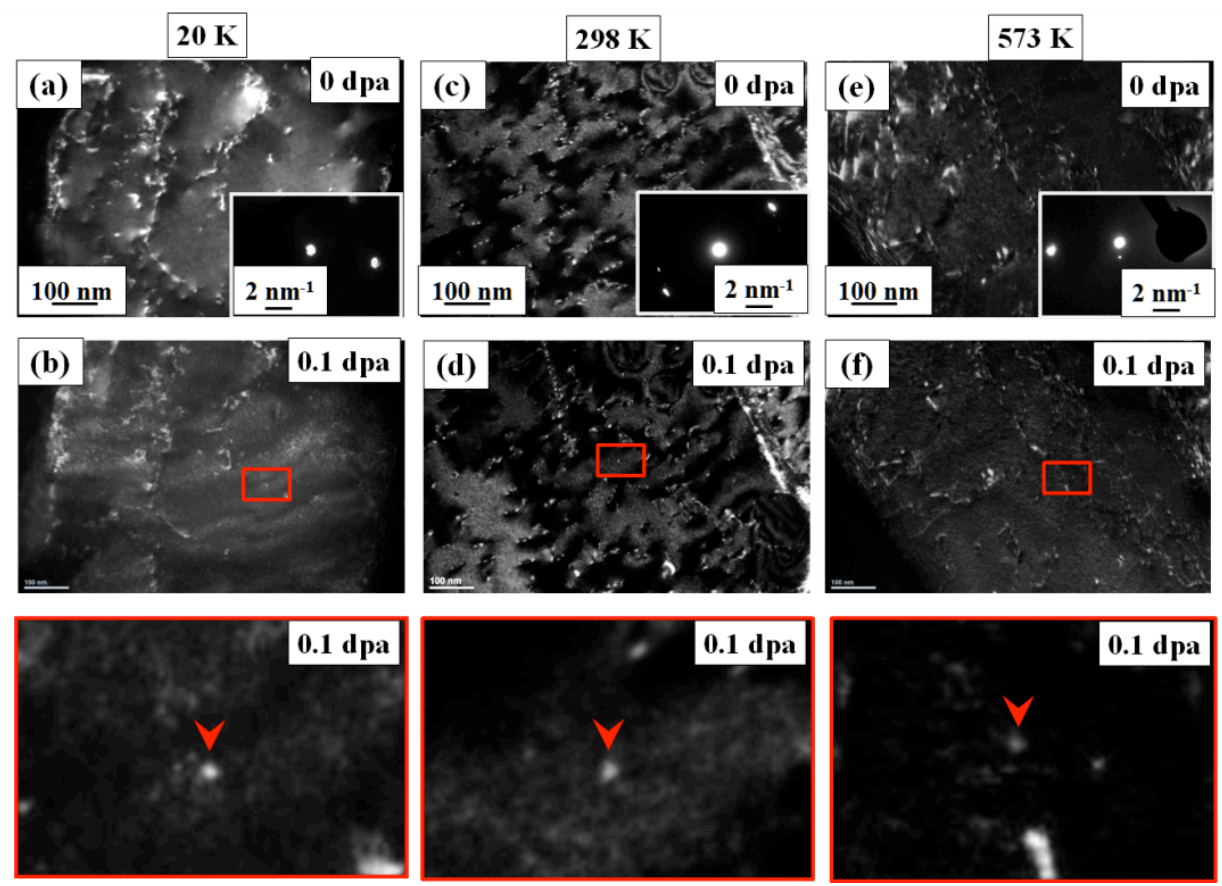

Figure 18. TEM images showing the initial stages of defect accumulation in HCM12A at 20, 298 and $573 \mathrm{~K}$. Insets of figures show the two beam condition and the close up view of the defect clusters. 
Frame-by-frame analysis of videos taken during irradiation showed that the white dots continuously appear and disappear in a time that is shorter than the time in between frames (normally $34 \mathrm{~ms}$ ). These white dots are thought to be small defect clusters $(2-5 \mathrm{~nm}$ in diameter), the nature of which (whether dislocation loops or 3-d clusters, and whether vacancy or interstitial) cannot be resolved. It is possible that these are small dislocation loops with Burgers vectors of either $<111>$ or $<100>$ as reported by Jenkins et al. Of these two types the $<111>$-type loops are more mobile compared to $<100>$ type loops.

Figures 19 and 20 show the video frames that capture the sudden appearance of five defect clusters during the irradiation of NF616 at $298 \mathrm{~K}$ and HCM12A at $20 \mathrm{~K}$. The defect formation was observed from one frame to the next at all irradiation temperatures $(20-573 \mathrm{~K})$, thus occurred in $34 \mathrm{~ms}$ or less, possibly in time frames comparable to the time required for cascade events to occur (1-5 ps).

(a)
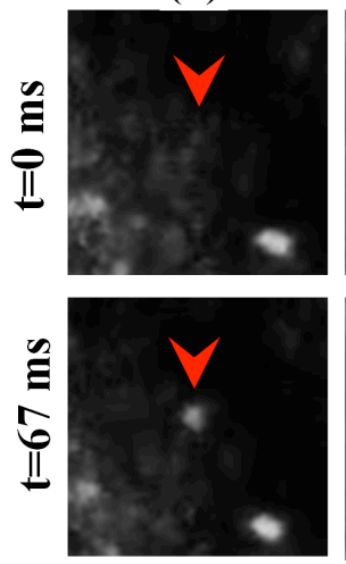

(b)


(c)
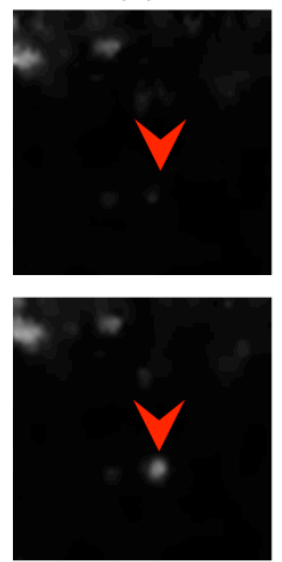

(d)
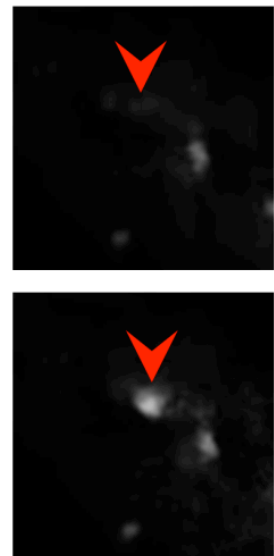

(e)
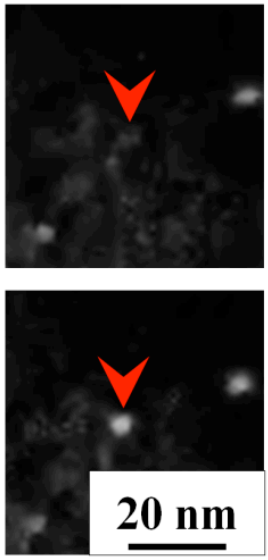

Figure 19. DF TEM images showing the appearance of five defect clusters in NF616 between video frames (in $67 \mathrm{~ms}$ or less ) at $298 \mathrm{~K}$.

(a)
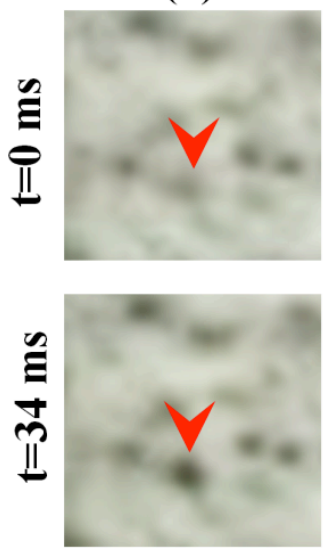

(b)


(c)
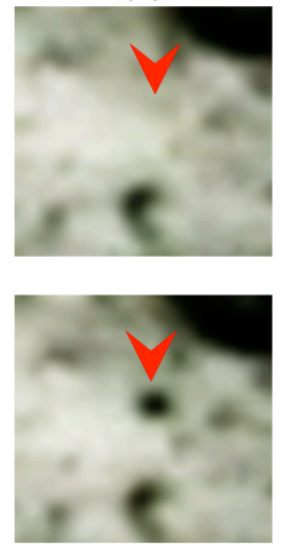

(d)


(e)
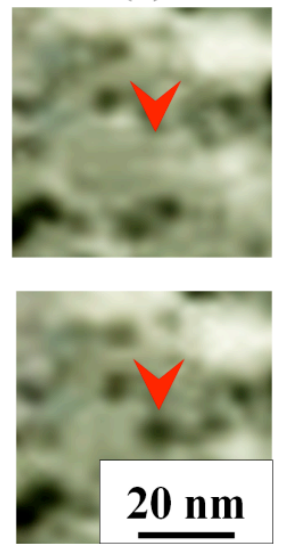

Figure 20. BF TEM images showing the appearance of five defect clusters in HCM12A between video frames (in $34 \mathrm{~ms}$ or less ) at $20 \mathrm{~K}$. 
In the heavy ion irradiations of bcc Fe, most point defects and their clusters are created in cascades created by energetic ion. The widely accepted picture of the distribution of defects in the final state of a cascade includes a vacancy-rich core surrounded by interstitials. In fcc materials such as $\mathrm{Cu}$ and $\mathrm{Ni}$, the vacancy-rich core of individual cascades was reported to create vacancy-type defects, that are visible in TEM even at doses below 0.0001 dpa, i.e., at doses which formation of defects by point defect migration and growth from a variety of displacement cascade events is not likely. On the contrary, single cascade events in bcc Fe initiated by high energy neutrons or ions do not reveal any TEM-visible damage, which was attributed to the relatively low intra-cascade clustering in Fe. Although collapse of individual, isolated cascades to visible defects in Fe did not occur under heavy-ion irradiations, visible damage was observed at high irradiation doses. The results of our in situ irradiation experiments are in agreement with this picture.

The fact that the formation of observable defect clusters in NF616 and HCM12A requires a finite irradiation dose, indicates that some cascade overlap and/or time for defect diffusion and reaction is necessary for TEM visible defect formation. Furthermore, analysis of the frame-by frame videos taken during irradiation shows that defects that were not present in a given frame were visible in the next, indicating that they were created in $34 \mathrm{~ms}$ or less. This is many orders of magnitude shorter than the time required for thermal diffusion to take place. It is important to note that defect formation was only observed under the ion beam at all temperatures. In addition defect creation took place at $20-50 \mathrm{~K}$, in fact with the highest efficiency, which indicates that defect creation in NF616 and HCM12A is a cascade-governed process.

What is the cascade process that enables formation of TEM-visible defects in NF616? Independent of the nature of the defects, the formation of TEM-visible defects in NF616 was governed by cascade events at all irradiation temperatures and necessitates spatial overlapping of cascades. Although it is not possible to perform inside-outside contrast for such small defects, it is expected, based on previous results from the literature to be interstitial. The defects in Fe and ferritic alloys produced by heavy ion and neutron irradiations were characterized as interstitial using TEM, and sub-nm vacancy clusters were in the form of nano-voids or loosely packed vacancy sponges as detected by positron annihilation studies. This is consistent with the MD studies which predicted a significantly higher fraction of intra-cascade clustering of interstitials compared to vacancies. In addition MD simulations showed that nanometer-sized interstitial clusters, containing as many as 100 interstitials exhibit fast one-dimensional motion towards their burgers vector with very low migration energies that are comparable to single interstitial migration energies. Therefore, mobile and sub-visible interstitial clusters may escape their collision cascade and interact with other defects created in other cascades. On the other hand, vacancy clusters need $\sim 50$ vacancies to collapse into a loop. The uncollapsed clusters do not glide in their glide cylinder and remain as three-dimensional vacancy clusters. In addition, threedimensional vacancy clusters move in a totally different time scheme.

Based on these observations, the following mechanism for defect TEM- visible defect formation can be proposed: As displacement cascades are generated in the material, their defect debris includes interstitial clusters in the sub-visible range, which can enhance microstructural evolution by eliminating the cluster nucleation phase. The TEM-invisible interstitial clusters with Burgers vector of $1 / 2<111>$ grow during irradiation by absorption of smaller clusters through one- 
dimensional motion in the direction of their Burgers vector. However, the defect motion is discontinuous since interstitial and substitutional solute atoms can trap defect clusters and immobilize them. On the other hand, cascade-induced shockwave created by high energy $\mathrm{Kr}$ ions may have enough energy to de-trap clusters, and unrestricted defect clusters interact with the defects in their glide cylinder until they are trapped again, as shown schematically in Figure 21. This motion allows interstitial loops to gather other interstitials along the way, recombine with vacancies or get annihilated at the sinks such as surface, grain boundaries and dislocations. Interstitial clusters with Burgers vector of $1 / 2<111>$ undergo a finite number of coalescence events which will eventually form defects that are observable in TEM $(>2 \mathrm{~nm})$.

Previous in situ heavy ion irradiation studies showed that a balanced population of interstitial clusters with Burgers vector of $1 / 2<111>$ and $<100>$ form in $\mathrm{Fe}-\mathrm{Cr}$ alloys at $298 \mathrm{~K}$. The formation of clusters with Burgers vector of $\langle 100\rangle$ is rather complicated since they are predicted to be sessile even at small sizes. Marian and Wirth proposed a mechanism for the formation and growth of defect clusters with $<100>$ Burgers. According to this mechanism, the $<100>$ cluster nuclei form through the direct interaction of mobile and sub-visible $1 / 2<111>$ clusters of similar size through the following reaction:

$$
\frac{1}{2}[111]+\frac{1}{2}[1 \overline{1} \overline{1}] \rightarrow[100]
$$

The reaction given in Equation (1) is a strain field mediated action but it can be activated by the thermal part of the cascade. Initially, the intersection of two $1 / 2<111>$ - type defect clusters forms $\mathrm{a}<100>110$ junction, which grows until the whole cluster is transformed. With increasing defect cluster size, $<100>110$ cluster rearrange itself to form pure-edge $<100>100$ defect clusters. The defect clusters with $<100>$ Burgers vectors are reported to have relatively low mobility and they reach TEM-visible size by absorption of small clusters with $1 / 2<111>$ Burgers vectors.



Figure 21. Schematic illustration of an interstitial prismatic loop moving in its glide cylinder while interacting with the defects till it is trapped by the solute atom. Blue region is the attractive interaction volume between the interstitial cluster and the solute atom.

It is also observed that under irradiation high fraction of these defect clusters exhibited sudden one-dimensional jumps and jerks, that is, some defect clusters move "or jump" along specific directions. 
Figures 22, 23 and 24 show the close-up view of defect clusters that exhibit the typical jumps which were consistently observed in NF616 and HCM12A below $573 \mathrm{~K}$. Video frames were extracted with $34 \mathrm{~ms}$ or $67 \mathrm{~ms}$ intervals, depending on the time resolution of the in situ irradiation movies. Note that the red arrows in Figures 22, 23 and 24 mark the initial position of the defects and yellow arrows mark the stationary defects in the close vicinity of displaced ones. Insets of Figures 22, 23 and 24 show the $<110>$ (g-vector) and $<211>$ directions.

The detailed TEM analysis showed that the jumps captured in the videos were one-dimensional and occurred in one of the $<211>$ directions, as shown in Figures 22, 23 and 24. Jumps occurred in + and - directions along $<211>$, and defects occasionally exhibited back-and-forth motion along $<211>$.

Previous studies on ion and electron beam-induced motion of small defect clusters $(<\sim 10 \mathrm{~nm})$ have shown that mostly clusters with Burgers vector of $1 / 2<111>$ undergo one-dimensional discrete jumps towards their Burgers vector in pure $\mathrm{Fe}$ and simple $\mathrm{Fe}-\mathrm{Cr}$ alloys. In our case, it was not possible to accurately determine the jump direction in our experiments, since both likely Burgers vectors $(<100>$ and $1 / 2<111>)$ project over the $<211>$ direction.

(a)
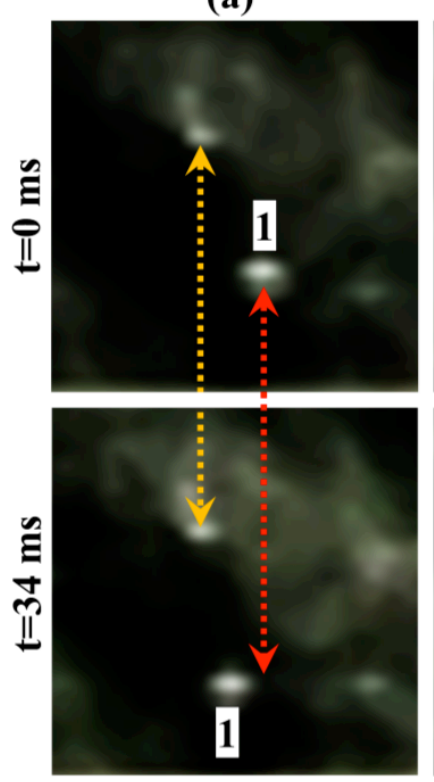

(b)
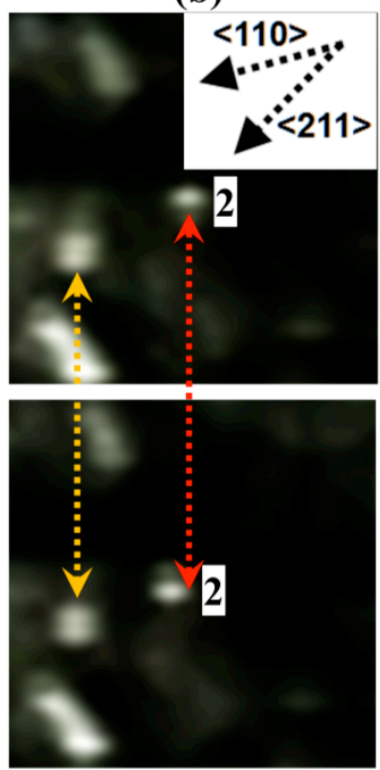

(c)

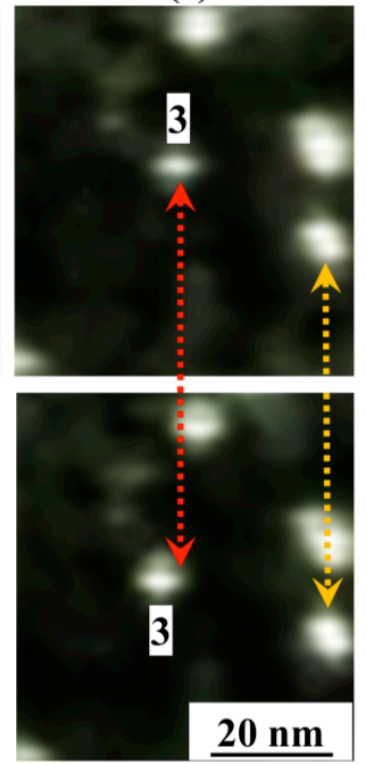

Figure 22. The successive video frames showing the close up view of the defects in NF616 irradiated to $\sim 0.9 \mathrm{dpa}$ at $373 \mathrm{~K}$. Defect cluters that exhibit jumps are tagged with numbers and red arrows point the initial position $(\mathrm{t}=0)$ of the defects in all images. Yellow arrows point the stationary defects in the images. 
(a)
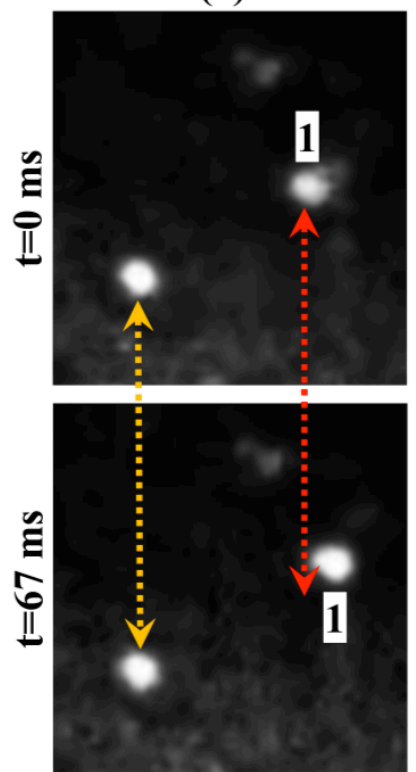

(b)
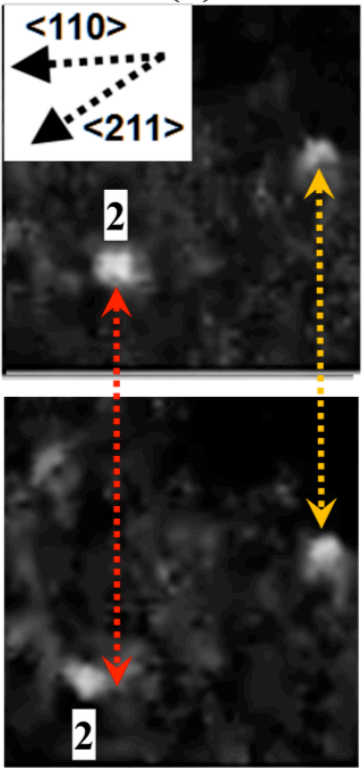

(c)
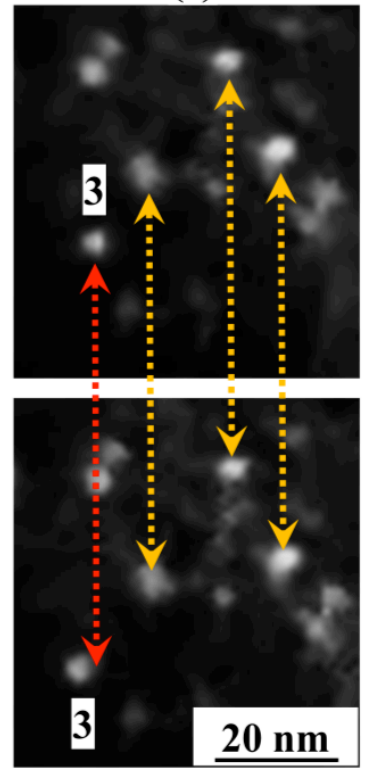

Figure 23. The successive video frames showing the close up view of the defects in NF616 irradiated to $\sim 1 \mathrm{dpa}$ at $298 \mathrm{~K}$. Defect cluters that exhibit jumps are tagged with numbers and red arrows point the initial position $(\mathrm{t}=0)$ of the defects in all images. Yellow arrows point the stationary defects in the images.

(a)

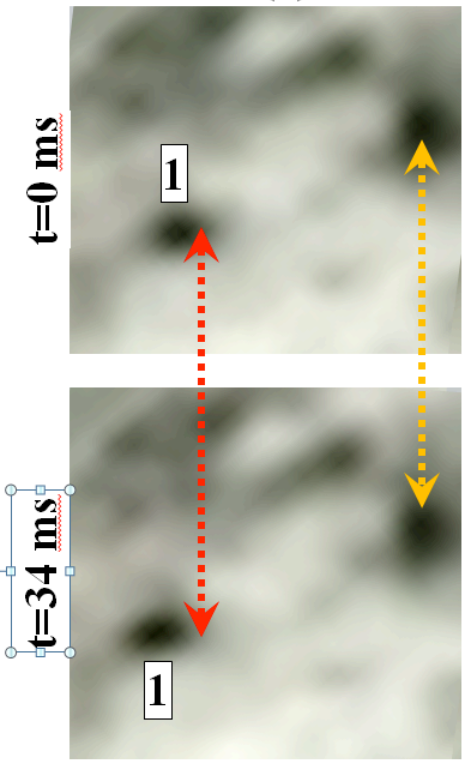

(b)

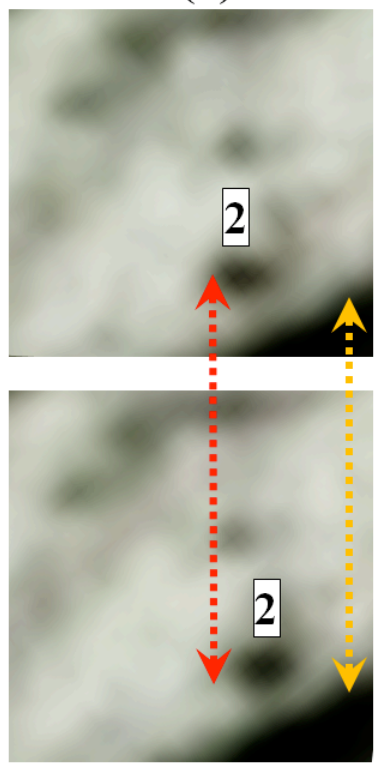

(c)

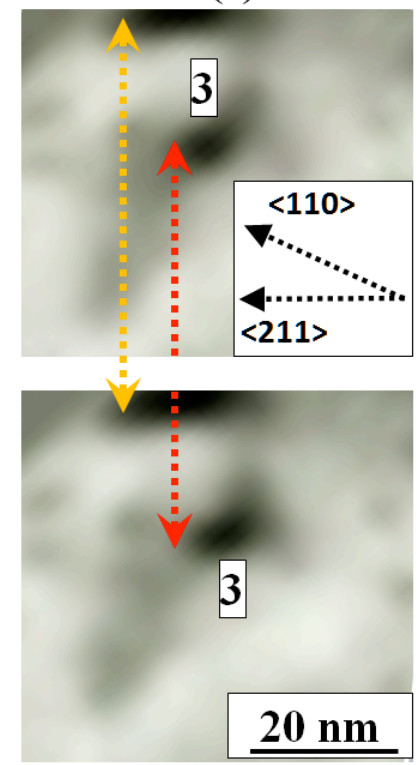

Figure 24. The successive video frames showing the close up view of the defects in HCM12A irradiated to $\sim 4 \mathrm{dpa}$ at $20 \mathrm{~K}$. Defect cluters that exhibit jumps are tagged with numbers and red arrows point the initial position $(\mathrm{t}=0)$ of the defects in all images. Yellow arrows point the stationary defects in the images. 
It is important to note that defect clusters displayed diverse behavior under irradiation. The number of defect jumps for each individual cluster changed considerably and a fraction of defect clusters did not exhibit jumps at all. Jenkins et al reported similar results for $\mathrm{Fe}$ and $\mathrm{Fe}-\mathrm{Cr}$ alloys regarding the motion of defect clusters: The frequency of one-dimensional jumps of defects in $\mathrm{Fe}-\mathrm{Cr}$ alloys varied considerably and a significant percentage of clusters were immobile.

Figure 1-15 shows the jump distance and direction of three defect clusters in NF616 irradiated to $\sim 1 \mathrm{dpa}$ at $373 \mathrm{~K}$. The frequency of defect jumps shown in Figure 25 range from 0.06 to $0.1 \mathrm{~s}^{-1}$. Furthermore, $52 \%$ the jumps were in the arbitrarily chosen "+" direction, which indicates the one dimensional jumps occur in both possible directions with similar probability. This clearly shows that the direction of jumps is not towards the hole in the wedge-shaped foil (+ direction), that is, in the direction of the thickness gradient. The distribution of jump distances measured for 52 defects in NF616 irradiated with $1 \mathrm{MeV} \mathrm{Kr}$ ions at $373 \mathrm{~K}$ is shown in Figure 26. The average jump distance is found to be $\sim 5 \mathrm{~nm}$. Note that, jumps smaller than $\sim 2 \mathrm{~nm}$ could not be measured because they are below the resolution limit of the captured video frames.
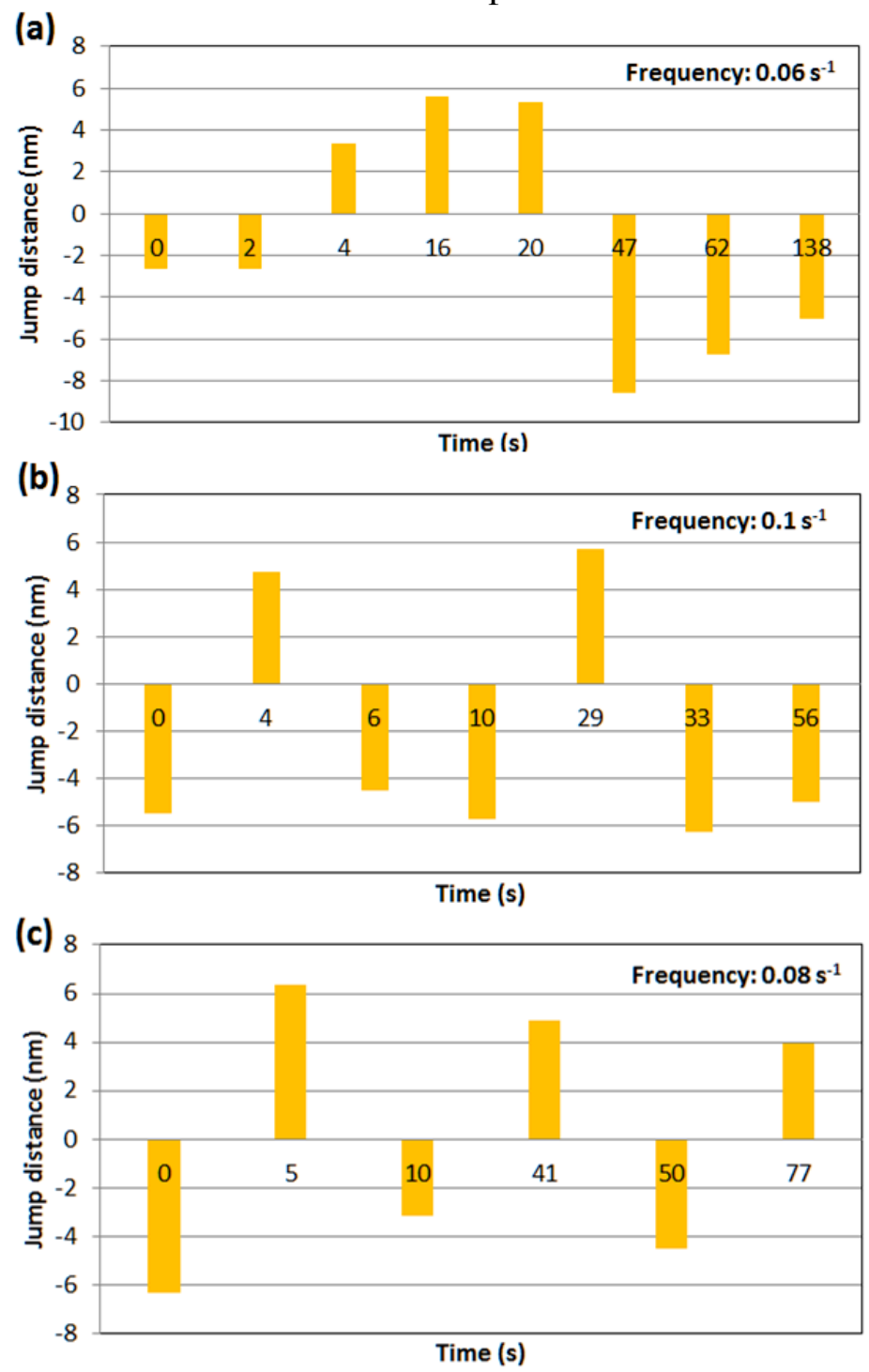

Figure 25. Jump distances of three defect clusters observed in NF616 irradiated with $1 \mathrm{MeV} \mathrm{Kr}$ ions at $373 \mathrm{~K}$. The average frequencies of defect jumps are noted on the plots. 


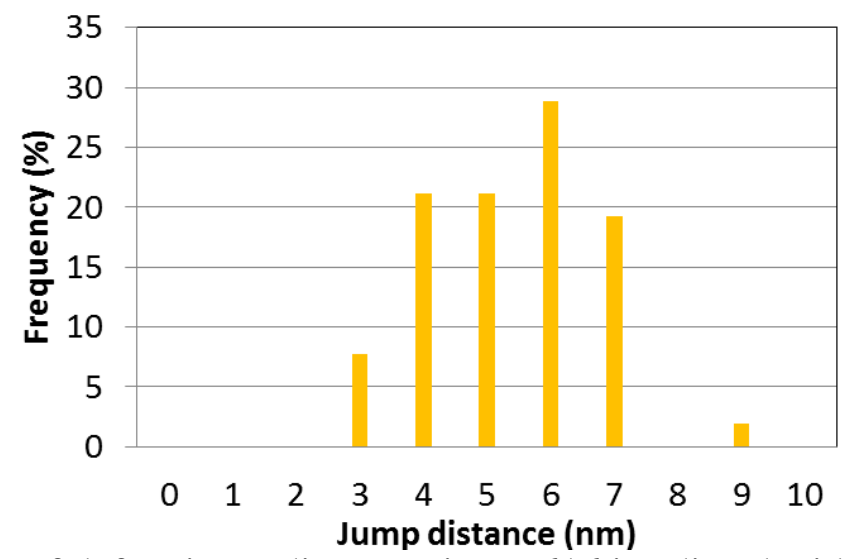

Figure 26. Distribution of defect jump distances in NF616 irradiated with $1 \mathrm{MeV} \mathrm{Kr}$ ions at 373 $\mathrm{K}$ with a bin size of $1 \mathrm{~nm}$. A total of 52 defects jumps were measured.

The facts that defect jumps were only observed under the ion beam and jumps were exceptionally fast (exceeding $250 \mathrm{~nm} \cdot \mathrm{s}^{-1}$ ) indicate that the cascade-induced shockwave created in the first picoseconds of the ion impact governs the defect motion in NF616 and HCM12A below $573 \mathrm{~K}$. It is important to note that defect jumps were observed at 20-50 K with a high frequency, which supports the cascade-induced defect motion mechanism since point defects and their clusters are thermally immobile at this temperature.

The defect cluster motion observed in NF616 and HCM12A under irradiation exhibits similarities to the one-dimensional defect motion in pure $\mathrm{Fe}$ and binary $\mathrm{Fe}-\mathrm{Cr}$ alloys which was reported to occur thermally or athermally (electron and ion beam-induced). The differences in the present observations with those reported in pure Fe and Fe-based commercial alloys can be ascribed to solute effects. For example pure Fe and model alloys were reported to exhibit discrete jumps under sub-threshold electron beam alone. Correspondingly, the average jump distance of defects decreases with increasing solute atom content. Jump distance was reported be $\sim 100 \mathrm{~nm}$ for high purity $\mathrm{Fe},<30 \mathrm{~nm}$ for the low-purity $\mathrm{Fe}$, and $<10 \mathrm{~nm}$ for NF616 and HCM12A (this study).

There is a very high density of substitutional $(\mathrm{Cr}, \mathrm{Mo}, \mathrm{V}, \mathrm{P}, \mathrm{Si}, \mathrm{Ni}, \mathrm{Mo}, \mathrm{Al}, \mathrm{W}, \mathrm{Mn}, \mathrm{Cu})$ and interstitional $(\mathrm{C}, \mathrm{N})$ solute atoms in NF616 and HCM12A, as given in Table 2. The interstitial solute atoms cause tetragonal distortions in bcc lattice and greatly reduce defect cluster mobility. In addition substitutional solute atoms decrease the mobility of defects in Fe to a lesser extent, depending on the misfit of the solute atom with the matrix. Furthermore, substitutional and interstitional solute atoms form mixed interstitial/substitutional atom atmosphere which can effectively pin the irradiation-induced defects. Certain combinations of interstitial and substitutional solute atoms were associated with anisotropic strain fields in bcc lattice, leading to strong elastic interactions between defects and solute atoms. Likewise, the pre-existing dislocation network in F-M steels is pinned by the solute atom atmosphere which greatly reduces the recovery processes (cross-slip and climb) at high temperatures, giving F-M alloys desirable elevated temperature properties. However, the high concentration of numerous alloying additions makes it hard to evaluate the individual and combined effect of the solute atoms on defect mobility. 
The above observations on one-dimensional sudden jumps below $673 \mathrm{~K}$ are consistent with the following mechanism of cascade-induced defect motion: First, the defect motion is analogous to the motion of sub-nm defect clusters with $<111>$ Burgers vectors described earlier in this text. The one-dimensional intermittent defect jumps in NF616 and HCM12A observed under irradiation suggest a mechanism by which the initially trapped defect clusters are set free by the necessary energy provided by the cascade impact. Once de-trapped, the defect clusters move with low activation energies within the glide cylinder until reaching the next trap in its glide cylinder. Figure 27shows the experimental setup for the in situ observations of sudden jumps and jerks in NF616 and HCM12A. The defect cluster (red loop) exhibits a jump between two traps (yellow discs), possibly solute atoms or complexes. Although the glide cylinder along <111> direction is tilted in this grain orientation (close to [111] zone axis), the projection of the defect motion appears one dimensional on the TEM screen as shown in Figure 27.

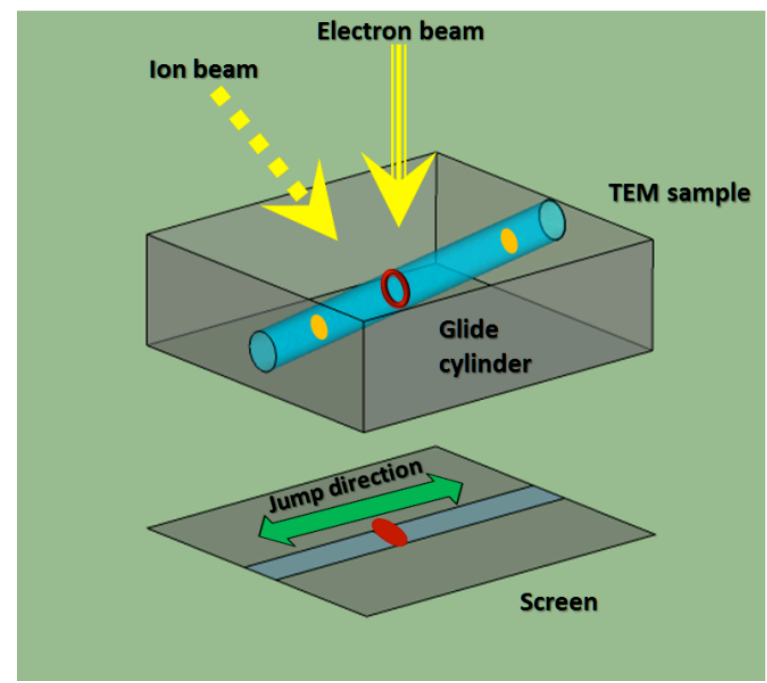

Figure 27. The schematic representation of the mechanism for the defect cluster (red loop) motion observed in HCM12A and NF616 below $673 \mathrm{~K}$ using the in situ TEM technique. The traps (solute atoms or complexes) are shown as yellow discs.

The overall density of defect clusters increased with dose and saturated around $6 \mathrm{dpa}$. The close up images of defect accumulation in NF616 and HCM12A between 20 and $573 \mathrm{~K}$ are shown in Figure 28.

In all irradiations, defects appeared as white dots in DF TEM images and the overall concentration of visible defects increased steadily with dose until it reached saturation Dynamic observations revealed that individual defect clusters appeared and disappeared during irradiation between 50 and $573 \mathrm{~K}$. This dynamic appearance and disappearance of defect clusters continued beyond the saturation dose. It is important to note that the defect formation process observed between 50 and $573 \mathrm{~K}$ was identical to the primary defect creation process, i.e., $2-5 \mathrm{~nm}$ defects formed only under the ion beam, as described earlier. Correspondingly, defect elimination was controlled by the ion beam and it was spontaneous (observed between frames, $34 \mathrm{~ms}$ or less). Interestingly, all the dynamic processes observed under irradiation between 50 and $573 \mathrm{~K}$ (defect appearance/disappearance and jumps) stopped as soon as the ion beam was turned off so that the defect cluster distribution that was present before the beam was turned off 
remains frozen. This indicates, these small defect clusters, once formed, are thermally immobile at least in the temperature regime $20-573 \mathrm{~K}$.

At saturation, a steady state is reached in which defects were eliminated and created at the same rates so that the density does not vary. In general, although the saturation densities were similar in this temperature range, they were slightly higher at lower temperatures.

In this temperature regime, the average diameter and size distribution of the irradiation-induced defect clusters did not vary with dose or temperature. Once the black dots were formed, no growth was observed during the irradiation period. The defect cluster density did not vary with foil thickness, indicating that the foil surface did not play a major role in the defect accumulation process.

At the end of each irradiation examination of the sample was performed using under focus/over focus contrast and no voids were observed at any temperature. Finally the irradiation-induced defect clusters showed no interaction with the pre-existing material microstructural features such as grain boundaries, carbides etc. Also no irradiation induced precipitates were observed in NF616 and HCM12A in this temperature range. 


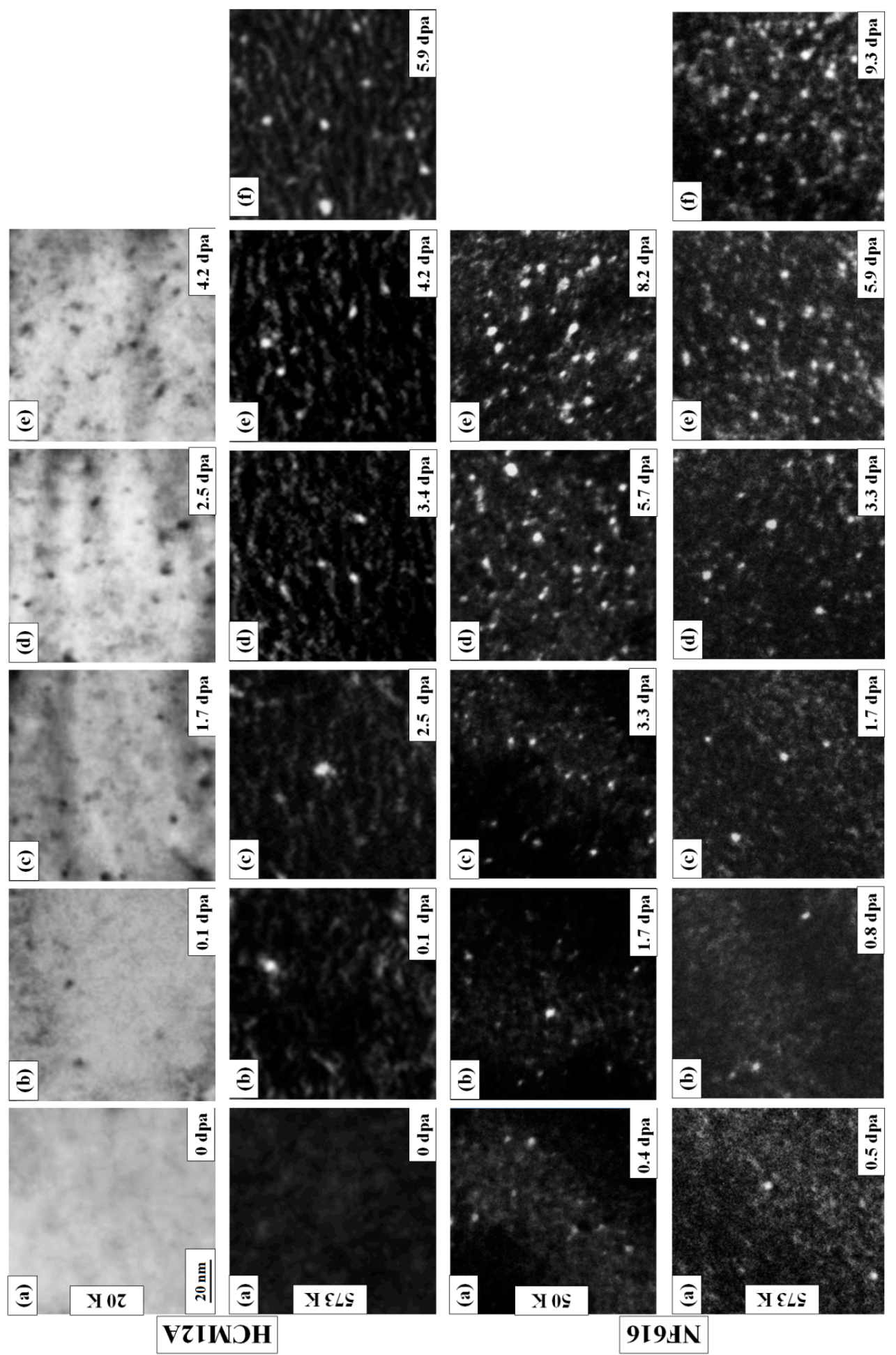

Figure 28. Observed microstructural evolution in NF616 and HCM12 as a function of irradiation dose and temperature. 


\section{High-temperature Region}

The maximum irradiation temperature of NF616 and HCM12A was 773 and $673 \mathrm{~K}$, respectively. Figure 29 shows a sequence of two-beam BF TEM images of the microstructure of NF616 and HCM12A irradiated at $673 \mathrm{~K}$. The initial defect formation process observed at these elevated temperatures was similar to the one observed at lower temperatures, i.e., 2-5 nm defects formed in $34 \mathrm{~ms}$ or less under the ion beam once the threshold dose for defect accumulation was reached. However, the dose for the onset of defect accumulation shifted to more than order of magnitude higher doses in both alloys at $673 \mathrm{~K}$. There was no visible damage in HCM12A and NF616 irradiated to $2.5 \mathrm{dpa}$ at $673 \mathrm{~K}$, as shown in Figure 29. This shift could be attributed to the lowering of the efficiency of defect formation with increasing temperature. The enhanced quenching of the cascade damage at low temperatures may assist in establishing the critical background density of sub-visible defects (intra-cascade interstitial clusters) necessary for the formation of visible defect clusters by a subsequent impact, as discussed earlier.

Interestingly sudden defect jumps were not observed in NF616 and HCM12A at $673 \mathrm{~K}$. The absence of one-dimensional defect motion can be attributed to the type of defect clusters that form at high temperatures in these alloys. As mentioned earlier in the text, previous studies showed the presence of a balanced population of $1 / 2<111>$ and $<100>$ type defect clusters in irradiated $\mathrm{Fe}-\mathrm{Cr}$ alloys at low temperatures. On the other hand, defect clusters with $<100>$ Burgers were mostly observed at high temperatures. Jenkins et al reported that the defects with $<100>$ to be sessile under heavy ion irradiation. While both $<100>$ and $1 / 2<111>$ defect clusters are pure-edge prismatic in nature, the $<100>$ loops necessitate a larger jump distance than $<111>$ loops. Marian and Wirth reported a high migration energy that exceeds $2.5 \mathrm{eV}$ for $<100>$ loops. Thus $<100>$ loops stay as immobile if not de-trapped by very high-energy cascades during irradiation.

At $673 \mathrm{~K}$, the defects in NF616 grew and coalesced under irradiation, which lead to larger average defect size and the lower defect density. The fact that there was no noticeable interaction of these relatively mobile defects with grain boundaries (no denuded zones) or with the preexisting dislocation network (no climb) at $673 \mathrm{~K}$, suggests that they have little long-range motion.

At high doses extended defect structures in NF616 were in the form of short segments aligned along 100 directions, as shown in Figure 29. Similar linear (or planar) defect superstructures were seen during irradiation of pure $\mathrm{Fe}$ at room temperature, although the defect clusters in that case were considerably larger, in agreement with the increased defect mobility in pure materials. The mechanism by which the alignment of defect structures occur is not known, however the mutual elastic interactions between clusters with $<100>$ Burgers vectors may lead to the formation of these superstructures, possibly to minimize the internal strain energy.

It is important to note that defect coalescence and growth only took place under the ion beam at $673 \mathrm{~K}$. Figure 30 shows the annealing of NF616 at $673 \mathrm{~K}$ alloy, which was initially irradiated at $298 \mathrm{~K}$ to $\sim 3 \mathrm{dpa}$. The microstructure of NF616 irradiated to $3 \mathrm{dpa}$ at $298 \mathrm{~K}$ was stable during annealing at $673 \mathrm{~K}$. There was no apparent change in defect size and density during annealing at $673 \mathrm{~K}$ for $1200 \mathrm{~s}$, as shown in Figure 30. This indicates the importance of the ion beam induced (athermal) contribution to defect mobility in NF616. 
At $673 \mathrm{~K}$, the microstructure evolution in HCM12A differed considerably from NF616 at high doses. 2-5 nm defect clusters, identical to the ones formed at low temperatures, were observed in HCM12A irradiated to high doses at $672 \mathrm{~K}$. It is evident from Figure 29 that smaller defect clusters were present in HCM12A with a significantly higher number density. This can be attributed to reduced mobility of defects in HCM12A due to the trapping of defects with the relatively high density of solute atoms in the alloy matrix.
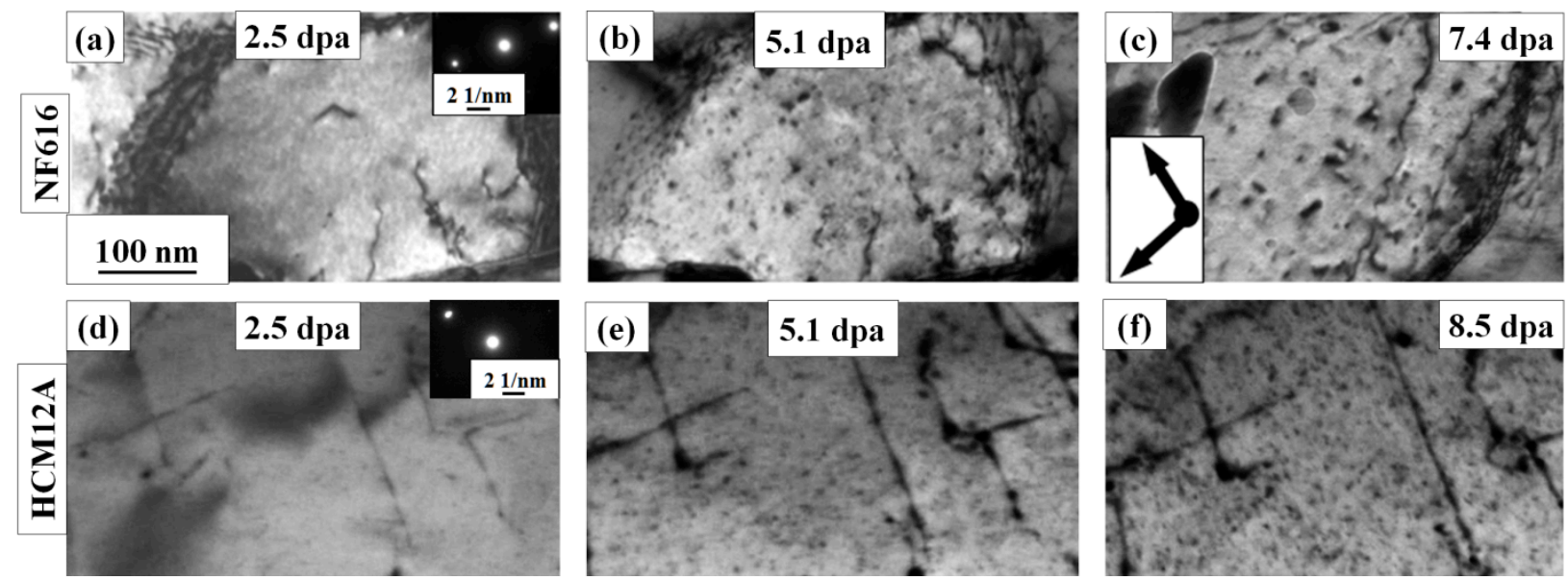

Figure 29. BF TEM images of microstructure evolution of NF616 and HCM12A under $1 \mathrm{MeV}$ Kr Irradiation at $673 \mathrm{~K}$.


Figure 30. DF TEM images of microstructure evolution of NF616 captured during annealing at $673 \mathrm{~K}$. NF616 was irradiated to $\sim 3 \mathrm{dpa}$ at room temperature prior to annealing.

Figure 31 shows the irradiation-induced microstructure evolution of NF616 at 773 K. At 773 K, 2-5 nm defects in NF616 started to appear at very high doses (3-4 dpa) and the frequency of defect formation per unit area was the lowest amongst all irradiations. The defect appearance was spontaneous, identical to the lower temperature irradiations. Once formed, defect clusters were immobile suggesting that defect trapping by solute atoms/complexes is still effective even at this high temperature. A fraction of these defect clusters grew in size for several seconds but all defect clusters eventually faded out slowly (on average over, 28 seconds) different from the 
defects observed at lower temperatures. Consequently, there was no net defect accumulation even at a high irradiation dose of $10 \mathrm{dpa}$, as shown in Figure 31.

The gradual shrinkage of defects at $773 \mathrm{~K}$ were unlikely to be lost to the foil surface since the irradiation is fully penetrating the foil, and the $2-5 \mathrm{~nm}$ defects near foil center cannot migrate to the surface easily, considering that they were immobile. Second, the defects we see appear (2-5 $\mathrm{nm}$ size) and then slowly fade and disappear over 10 to 70 seconds are rather few and certainly do not represent a dominant effect at $773 \mathrm{~K}$. Typically, TEM-visible defects are lost to the surface in less than $0.1 \mathrm{sec}$ therefore this slow fading away over 10 to 70 seconds is not consistent with loss to a surface. Based on these observations, it can be suggested that most defect clusters are not making it to TEM-visible sizes in general and that a steady state of production, perhaps clustering and trapping to small sizes, and annihilation by recombination is operating at $773 \mathrm{~K}$. In that case, the defects we observed that appeared suddenly and faded out gradually represent a fluctuation in this behavior.

At $773 \mathrm{~K}$, no resolvable loops, voids or irradiation-induced precipitates were observed. Also, no interaction of the irradiation induced defects with the pre-existing microstructure (dislocation network, grain boundaries) in the form of denuded zones, or dislocation climb was seen.
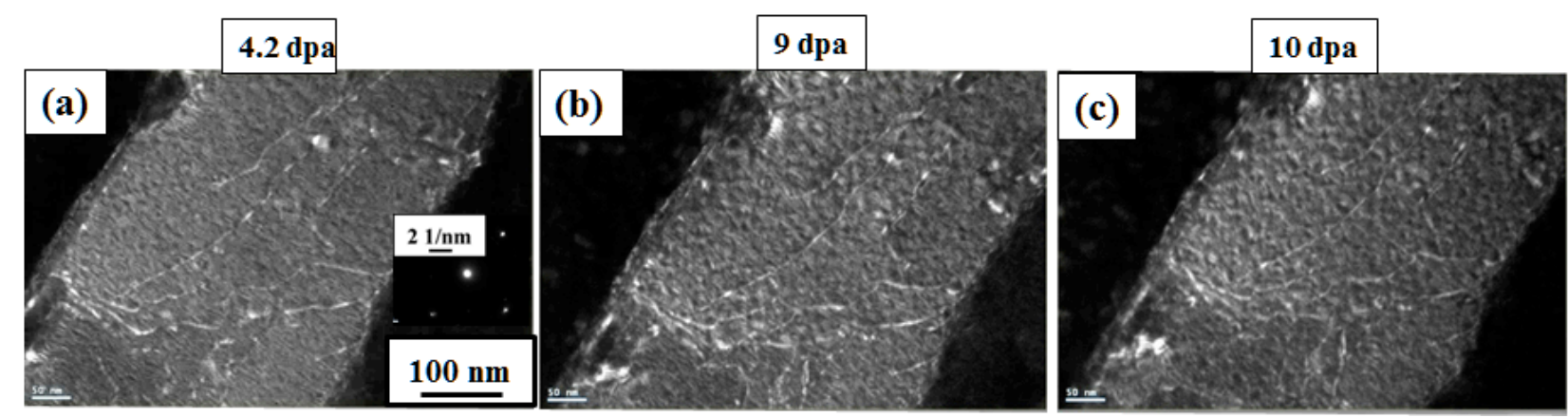

Figure 31. DF images of NF616 irradiated to 4, 9 and 10 dpa at $773 \mathrm{~K}$.

During the irradiations, the areas for observation in NF616 were significantly larger than those of the HCM12A due to the high pre-existing dislocation density in the alloy. This allowed to analyze a high number of defects in NF616, thus improving statistics. Because of the limited sampling area in HCM12A, quantitative analysis performed on TEM images of NF616 is given here.

Quantitative analysis was performed on TEM images of NF616 to determine defect size and density by manually counting a total number of $\sim 2 \times 10^{4}$ defect clusters using Image J software. The areal density of defect clusters for the irradiations at 50,473, 573,674 and $773 \mathrm{~K}$ were counted manually from the micrographs using ImageJ and the results are plotted in Figure 32 versus irradiation dose. Note that, the areal density $\left[\mathrm{nm}^{-2}\right]$ can be converted to a volumetric density $\left[\mathrm{nm}^{-3}\right]$ if the foil thickness is known. Assuming a foil thickness of $100 \mathrm{~nm}$, the top density of $4.6 \times 10^{-4} \mathrm{~nm}^{-2}$ would become $4.6 \times 10^{-6}\left[\mathrm{~nm}^{-3}\right]$. When the defect was not clearly identified, the feature was counted as $1 / 2$ defect. Dislocation loops from a particular family of Burgers vectors are expected to form with equal probability in all equivalent orientations. For a 
given two beam imaging condition, only a fraction may be visible, depending on the specific visibility criterion. Since no correction was made to the measured defect density values to take into account the invisibility criterion, the reported defect densities are low by a factor of 2 to 3 . Assuming that the defects are of the same type the densities will be lowered by the same factor in all micrographs. Figure 32 shows a steady increase in defect density and saturation around 6 dpa between 50 and $673 \mathrm{~K}$. Furthermore, saturation defect density decreases with increasing temperature, as shown in Figure 32. Note that, there was no net defect accumulation at $773 \mathrm{~K}$. Figure 32 shows the average defect cluster size as a function of dose at 50, 473, 573 and $673 \mathrm{~K}$. Average defect cluster size values and defect size distributions are shown only above $2.5 \mathrm{dpa}$ because of the poor statistics due to the small number of defects at early doses. The average defect size does not change significantly with dose between 50 and $573 \mathrm{~K}$ and there is a significant in defect size at $673 \mathrm{~K}$, as shown in Figure 33. The defect cluster size distributions are plotted in Figure 32, for the 50, 473 and $573 \mathrm{~K}$ irradiations, respectively. The defect cluster size distributions vary little during irradiation in this temperature regime, especially after saturation. Consequently, no significant change is seen between the defect cluster size distributions at $50 \mathrm{~K}$ and $573 \mathrm{~K}$.


Figure 32. (a) Defect cluster areal density of NF616 as a function of dose between 50 and $773 \mathrm{~K}$. (b) Average defect cluster size of NF616 as a function of dose between 50 and $773 \mathrm{~K}$. 

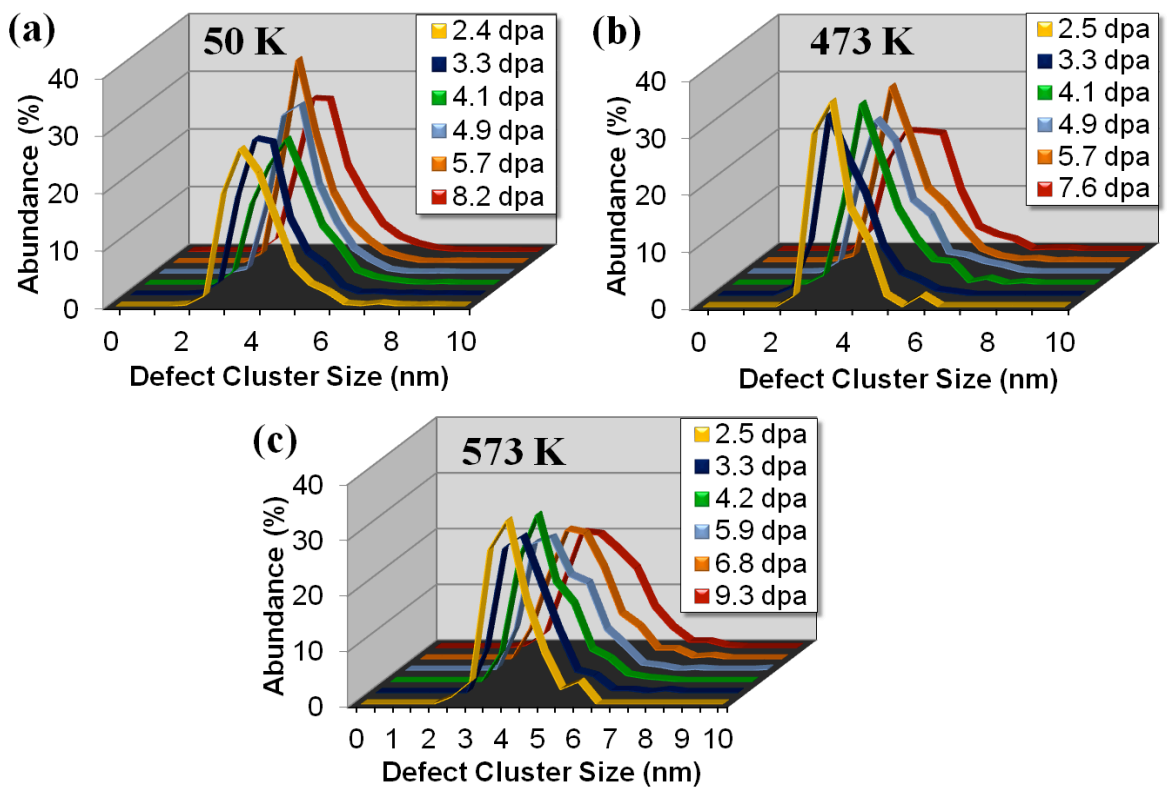

Figure 33. Size distribution of defect clusters in NF616 irradiated with $1 \mathrm{MeV} \mathrm{Kr}$ ions at 50, 273 and $373 \mathrm{~K}$ as a function of dose, using a bin size of $0.5 \mathrm{~nm}$.

Experimental Results - Characterization and Irradiation of ODS steels:

The nominal compositions of the two alloys of interest (18CrODS and 9CrODS) are listed in Table 3 .

Characterization methods: In order to identify the matrix and nano-particle composition, phase, and relative abundance the alloys were analyzed using Synchrotron X-ray diffraction analysis at the Advanced Photon Source at Argonne National Laboratory. The synchrotron source allows for higher energy photons and higher brilliance than the typically utilized $\mathrm{Cu}-\mathrm{K} \alpha$ ray sources in typical diffractometer, which enables observance of signal from low volume fraction precipitates. The diffraction spectrum was taken using $15 \mathrm{keV}$ photons. Also, in order to further substantiate the phases identified, chemical extractions of precipitates were characterized using Cuk $\alpha$ radiation. Finally, bulk samples of $18 \mathrm{Cr}$ ODS were subject to $\mathrm{CuK} \alpha \mathrm{XRD}$ analysis in order to compare data acquisition techniques.

For precipitate extraction, the metallic matrices of the specimens were dissolved in a Berzelius type solution. Alloy samples of about $2 \mathrm{~g}$ were weighed and the matrix dissolved at room temperature. Mixing was aided by an electromagnetic stirrer. After filtering through a $20 \mathrm{~nm}$ mesh filter, the precipitates were cleaned with a hydrochloric wash, and dried for two hours at $105{ }^{\circ} \mathrm{C}$.

The extractions were then analyzed using a Rigaku Ultima IV Powder X-ray diffractometer (CuKa radiation) with a diffracted beam graphite monochromator in order to obtain a diffraction pattern without the obstruction of peaks from the matrix. Additionally, bulk samples taken from both the transverse and longitudinal directions were analyzed using the same diffractometer. 
The XRD spectra were analyzed using PDF 2 ICDD database, Peak Fit, and SIeve data analysis software and the results compared with findings from the Synchrotron XRD data in order to substantiate peak identifications and methods.

TEM and SEM analysis was conducted using thin foil samples of the two alloys. In order to form thin foils from the bulk samples, the alloys were first cut and polished down to a thickness of about $1 \mu \mathrm{m}$ using a polishing wheel and increasingly fine grit polishing sheets. The foils were then punched into discs approximately $3 \mathrm{~mm}$ in diameter, and electropolished using an electrolyte solution of methanol and perchloric acid. The samples were characterized using TEM to observe nanoparticle size and distribution and FESEM coupled with EDS analysis for chemical analysis. Comparison/correlation of the composition analysis from Synchrotron XRD and EDS analysis was made in order to consolidate and verify the presence of oxide and carbide nanoparticles in specific phases dispersed within the Fe-Cr matrices.

Table 3. ODS Alloy Composition by wt $\%$.

\begin{tabular}{|l|l|l|}
\hline Element & $\begin{array}{l}\text { Fe- } \\
\mathbf{1 8 C r}\end{array}$ & $\begin{array}{l}\text { Fe- } \\
\text { 9Cr }\end{array}$ \\
\hline $\mathrm{Fe}$ & $\mathrm{Bal}$. & $\mathrm{Bal}$. \\
\hline $\mathrm{Cr}$ & 18 & 8.67 \\
\hline $\mathrm{W}$ & 1 & 1.96 \\
\hline $\mathrm{Ti}$ & 0.25 & 0.23 \\
\hline $\mathrm{Mn}$ & 0.3 & 0.05 \\
\hline $\mathrm{Si}$ & 0.3 & 0.048 \\
\hline $\mathrm{Ni}$ & 0.19 & 0.06 \\
\hline $\mathrm{C}$ & 0.027 & 0.14 \\
\hline $\mathrm{P}$ & - & $<0.005$ \\
\hline $\mathrm{S}$ & - & 0.003 \\
\hline $\mathrm{N}$ & - & 0.017 \\
\hline $\mathrm{Ar}$ & - & 0.004 \\
\hline $\mathrm{Y}$ & - & 0.27 \\
\hline $\mathrm{O}$ & 0.11 & 0.14 \\
\hline $\mathrm{Y} 2 \mathrm{O} 3$ & 0.56 & 0.34 \\
\hline Ex. O & - & 0.07 \\
\hline
\end{tabular}

18Cr ODS is manufactured with a ferritic structure which has been shown to have high corrosion resistance due primarily to high-chromium content and a fine grain structure.

Characterization: XRD analysis of $18 \mathrm{Cr}$ ODS revealed a strong resemblance between the synchrotron spectrum of the bulk sample and the $\mathrm{Cu}-\mathrm{K} \alpha$ spectrum of the extraction as can been seen on fig. 34. The phases identified through analysis are the BCC Matrix $(\mathrm{Fe}, \mathrm{Cr})$, in addition to an Iron Chromium Carbide of the form $\mathrm{M}_{23} \mathrm{C}_{6}$ and Yttrium Titanium Oxide of the form $\mathrm{Y}_{2} \mathrm{Ti}_{2} \mathrm{O}_{7}$. Peak Fit analysis of these identifications showed that the peaks from $\mathrm{Y}_{2} \mathrm{Ti}_{2} \mathrm{O}_{7}$ and $(\mathrm{Cr}$, $\mathrm{Fe})_{23} \mathrm{C}_{6}$ are of the highest volume fraction in the sample as these two phases alone account for all but a few minor peaks. FE-SEM analysis of $18 \mathrm{Cr}$ ODS allowed substantiation of XRD results 
through imaging of nanoparticles along with EDS analysis. SEM analysis revealed the presence of Al-O, Al-Ti-O, Ti-O, Ti-O, and Ni-Mo particles in 18Cr ODS and substantiated the presence of $(\mathrm{Cr}, \mathrm{Fe})_{23} \mathrm{C}_{6}$ and $\mathrm{Y}_{2} \mathrm{Ti}_{2} \mathrm{O}_{7}$ identified through XRD analysis. Figure 34 shows the identified phases overlaid on the extraction diffraction spectrum for $18 \mathrm{Cr}$ ODS. BCC $\mathrm{Y}_{2} \mathrm{O}_{3}$ is seen represented in the XRD pattern in low volume fraction. However, while the presence of $\mathrm{Y}_{2} \mathrm{O}_{3}$ seemed to help get a better fit of the peaks collected in the XRD spectrum, SEM analysis did not confirm the presence of pure Yttria, as all Yttrium and Oxygen containing particles observed in $18 \mathrm{Cr}$ ODS by EDS were in compounds with either titanium or aluminum.

The particle dispersion in 18Cr ODS, observed by SEM and EDS in general consists of three particle types analysis (illustrated in Fig 35, 36, 37): large (up to the $\mu \mathrm{m}$ range) rounded particles appearing to be Al- containing oxides, strings of small, rounded particles observed to be Ti- rich particulates with sizes of $100 \mathrm{~nm}-200 \mathrm{~nm}$ or smaller, and smaller $(<50 \mathrm{~nm})$ particles containing $\mathrm{Y}-\mathrm{Ti}-\mathrm{O}$ (corresponding to the $\mathrm{Y}_{2} \mathrm{Ti}_{2} \mathrm{O}_{7}$ particles identified from XRD analysis). There seemed to be a correlation between size of precipitates and $\mathrm{Ti}$ and $\mathrm{Al}$ content: $\mathrm{Ti}$ seems to promote smaller size precipitates whereas Al tends to be found in larger precipitates. There also seemed to be an apparent accumulation of particles at some grain boundaries while others were free of precipitates.

In-Situ Irradiation: 18Cr ODS samples were irradiated with $1 \mathrm{MeV} \mathrm{Kr}$ ions to 10, 30, and 52 dpa at $25^{\circ} \mathrm{C}$, to $30 \mathrm{dpa}$ at $300^{\circ} \mathrm{C}$, and to $20 \mathrm{dpa}$ at $500^{\circ} \mathrm{C}$. Overall, in-situ observations illustrate the stability of nanoparticles up to $20 \mathrm{dpa}$ at $500^{\circ} \mathrm{C}$. The imaging conditions begin to deteriorate after 10dpa, however it is evident between 15 and 20 dpa that the nanoparticles within the grains are still present and do not appear to have changed in size or dispersion. The $18 \mathrm{Cr}$ ODS samples irradiated up to $52 \mathrm{dpa}$ at $25^{\circ} \mathrm{C}$ showed no apparent change in the size or shape of the nanoparticle. However, there is observed amorphization of some particles at the lower temperatures.

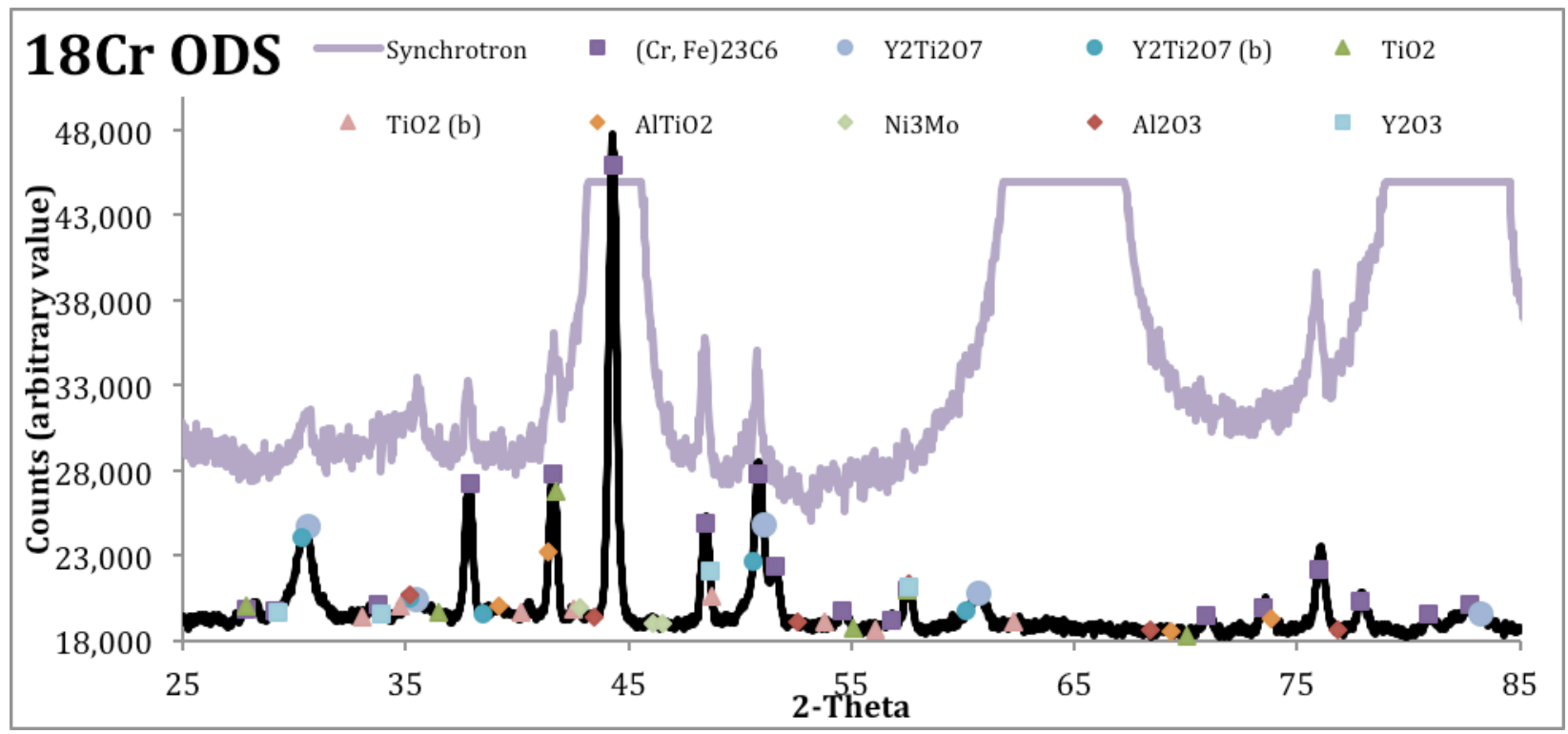

Figure 34. 18Cr ODS CuK $\alpha$ X-ray diffraction spectrum of nanoparticles after chemical extraction from the alloy matrix with peak locations labeled for all phases showing their presence in the alloy represented in the collected data; Synchrotron data is superimposed, showing similar second-phase peaks but also including large matrix peaks. 




Figure 35: a):18Cr: Y-Ti-O in matrix; b): Y-Ti-O after extraction

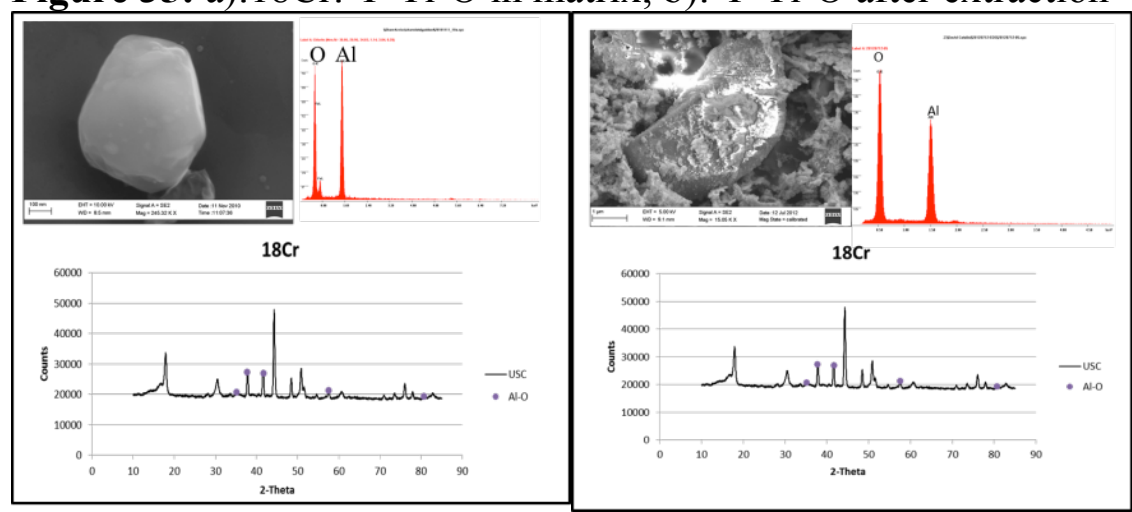

Figure 36. a).18Cr: Al-O in matrix; b): Al-O after extraction

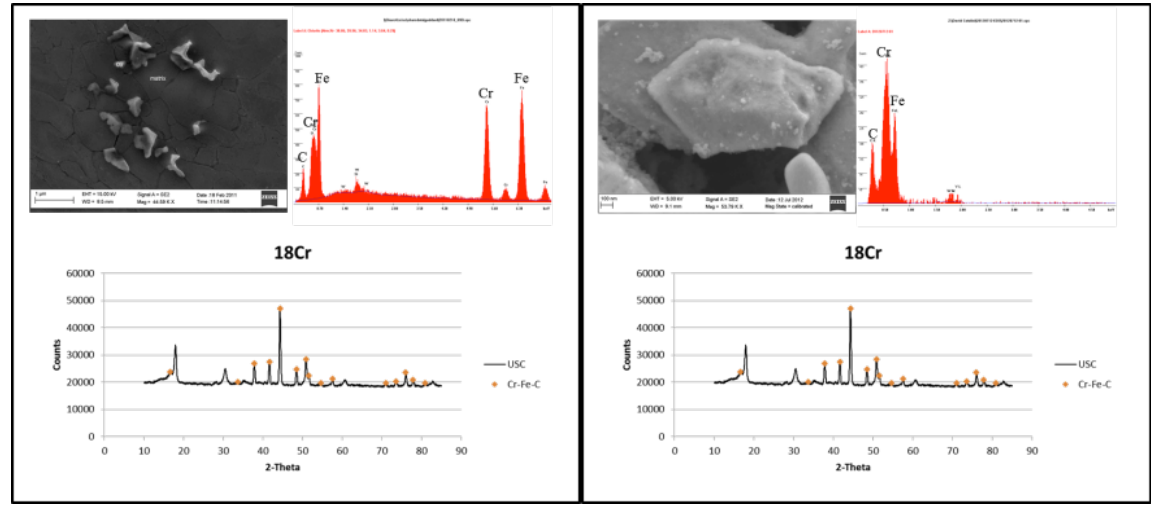

Figure 37. a): 18Cr: Cr-Fe-C in matrix; b): 18Cr: Cr-Fe-C after extraction

9Cr ODS is fabricated with a largely martensitic structure, however the addition $\mathrm{Y}_{2} \mathrm{O}_{3}$ during fabrication leads the formation of a non-equilibrium phase, designated as the residual ferrite, which serves to greatly enhance several vital mechanical properties. In 9Cr ODS the grain size is observed by SEM images to be also less than $1 \mu \mathrm{m}$ in the direction of observation. The combination of small oxide particles and the residual $\alpha$-ferrite phase serve to produce the observed very fine grain structure without the additional step of recrystallization. 
Characterization: Synchrotron XRD of the bulk samples and $\mathrm{Cu}-\mathrm{K} \alpha$ diffraction of the precipitate extractions showed similar spectra, however the extraction allows for better peak identification as there was less obstruction in the pattern from the large matrix peaks.

The phases identified through XRD analysis in the 9Cr ODS were mainly: Chromium Iron Tungsten Carbide $\left((\mathrm{Cr}, \mathrm{Fe}, \mathrm{W})_{23} \mathrm{Fe}_{21} \mathrm{~W}_{2} \mathrm{C}_{12}\right)$, Chromium Iron Carbide $\left((\mathrm{Cr}, \mathrm{Fe})_{23} \mathrm{C}_{6}\right)$, Titanium Oxide (TiO), Titanium Nitride (TiN), and Yttrium Titanium Oxide $\left(\mathrm{Y}_{2} \mathrm{Ti}_{2} \mathrm{O}_{7}\right)$. SEM and EDS analysis substantiated the presence of these Iron Chromium carbides as well as the presence of Titanium Oxide, Titanium Nitride, and Yttrium Titanium Oxide. $\mathrm{Y}_{2} \mathrm{O}_{3}$ could not be detected in the spectra and the $\mathrm{Y}_{2} \mathrm{Ti}_{2} \mathrm{O}_{7}$ phase was represented in the XRD pattern, however the relative intensity of the correlating peaks were very low in both the extraction and synchrotron data. This could be due to the limitations associated with the methods to detect particles of less than a few nms as discussed later. Indeed, the low representation of Y-Ti-O phases in the XRD spectra may not be representative of the actual dispersion in the alloy, as the filter used after extraction had a grid spacing of $20 \mathrm{~nm}$, so particles smaller than $20 \mathrm{~nm}$ (most $\mathrm{Y}_{2} \mathrm{Ti}_{2} \mathrm{O}_{7}$ particles) are mostly lost after filtering and are only captured if they settle on top of larger particles.

The low intensity representation of $\mathrm{Y}_{2} \mathrm{Ti}_{2} \mathrm{O}_{7}$ and unability to detect $\mathrm{Y}_{2} \mathrm{O}_{3}$ in the synchrotron pattern does not allow to exclude these two families of oxides from the dispersion in the ODS steel. In fact, this may well indicate that signal from very small particles $(<20 \mathrm{~nm})$ was not picked up (to a detectable level on the spectrum) through bulk synchrotron analysis, and only larger particles are clearly represented in the resultant diffraction pattern. PeakFit analysis (through deconvolution of peaks) of the phases identified revealed that the carbides are the highest volume fraction within the extractions, however the presence of all the smaller oxide precipitates identified through SEM are reflected in the pattern, often under convoluted peaks.

On particle size and chemical composition correlation, EDS analysis of nanoparticle dispersion in 9Cr ODS reveals that the largest particles are Al- containing oxides while smaller $(<20 \mathrm{~nm})$ more rounded particles tend to be Y- containing. Using Energy Filtering-TEM (in Fe-M jump ratio mode) and Atom Probe Tomography (APT) for further analysis could reveal a more accurate approximation of the smaller nanoparticles (of sizes less than $5 \mathrm{nms}$ ). The TEM observations showed that some grain boundaries are decorated with precipitates as was seen in $18 \mathrm{Cr}$ ODS. Also, larger elongated particles were seen primarily accumulated along grain boundaries have been observed to be $\mathrm{Cr}$ - carbides.

In-situ Ion Irradiations: Similar irradiations were conducted in 9Cr ODS, large particles, likely carbides, appear to undergo increasing amorphization at successive doses at room temperature while very small particles and mid-range particles are stable at doses up to 10dpa as evidenced by TEM. Amorphization of larger nanoparticles under irradiation has been observed also in a study by M.-L Lescoat, I. Monnet, et. al. in the irradiation of DY and EM10 ODS alloys for low energy ion irradiations conducted below $500^{\circ} \mathrm{C}$. Additionally, EFTEM energy filtering maps of samples irradiated at $500 \mathrm{C}$ show that very small particles less than $10 \mathrm{~nm}$ are still present but appear to be enriched with chromium. 


\section{Results: Computational Modeling}

Cluster Dynamics Modeling of Defect Cluster Evolution:

The development of the visible defect clusters has been examined using cluster dynamics models in an attempt to understand the kinetics that drive the formation and growth of extended defects. This model employs a set of spatially dependent diffusion-reaction equations, each of which governs the concentration of a corresponding cluster size. For a cluster with $n$ members, the equation takes the form

$$
\frac{\partial C_{n}(t)}{\partial t}=D \nabla^{2} C_{n}(t)-F_{n}(t)+G_{n}(t)+\gamma_{n}
$$

where $\mathrm{D}$ is the diffusion coefficient for a cluster containing $\mathrm{n}$ defects, $\mathrm{F}_{\mathrm{n}}$ is the volumetric rate of loss of clusters with size $\mathrm{n}$ as a result of absorption at fixed sinks like the free surface or dislocations and as a result of clustering reactions, $G_{n}$ is the production rate of clusters of size $n$ as a result of reactions between clusters, and $\gamma_{n}$ is the production rate of clusters directly from displacement cascades. The reaction rate between two clusters with sizes that sum to $\mathrm{n}$ must be evaluated over all possible combination to determine the reaction production term $\mathrm{G}_{\mathrm{n}}$. The reaction rate between two cluster species of size $\mathrm{i}$ and $\mathrm{j}$, respectively, is defined by standard chemical rate theory as:

$$
R_{i j}(t)=k_{i j} C_{i}(t) C_{j}(t)
$$

where the rate constant $\mathrm{k}_{\mathrm{ij}}$ is a function of the interaction geometry and mobility of the reacting clusters. The spatial dependence in the system of equations defined by Eq. (2) above is necessary because of the strong sink effect of the free surfaces of the thin films used for in-situ ion irradiation studies. The front and back surfaces in the simulation are set to black absorbers, which maintain zero cluster concentrations. Finite differences are used to discretize the spatial derivatives.

The rate of production due to cascades, $\gamma_{n}$, has been defined by taking into account the $1 \mathrm{MeV}$ $\mathrm{Kr}$ ion flux, the depth distribution of primary knock-on atom energies obtained from SRIM calculations, and the cascade clustering observed in molecular dynamics (MD) simulations in body-centered cubic iron as a function of PKA energy that were performed by Stoller. The MD studies indicate that cascades generate a significant number of small clusters in addition to point defects. Cluster dynamics models allow this phenomenon to be addressed explicitly by including source terms in the equations for these small cluster sizes. A parameterization of the cascade database provided by Stoller has been developed that considers both the in-cascade recombination that reduces the overall defect production, as well as the self-clustering that can produce a fraction of interstitial clusters with as many as 20 members and vacancy clusters with as many as 9 members.

Direct production of clusters in cascade debris in this size range is not nearly large enough to meet the visibility criteria but may provide nucleation sites for larger dislocation loops. The density of these nucleation sites is a strong function of the mobility of small dislocation loops, which can be expected to be similar to the observed mobility of visible loops. MD simulations 
clearly show that small interstitial clusters are effectively prismatic dislocation loops that can migrate easily along the glide direction with activation energies on the order of $0.1 \mathrm{eV}$ or less. However, experimental studies using in-situ TEM do not indicate that the mobility of visible loops is this high, and instead suggest that loop mobility is limited by trapping interactions with interstitial impurity atoms which results in an effective activation energy for loop migration on the order of $1.3 \mathrm{eV}$. The MD studies also indicate that interstitial cluster mobility is independent of size, as do the in situ TEM studies.

The in-situ observations presented here show loops that are immobile thermally at $573 \mathrm{~K}$ and below, but begin to exhibit sudden jumps under irradiation when the $\mathrm{Kr}$ ion beam is on. While possible ion-beam induced effects relate to both displacement cascade overlap leading to cluster reactions (cascade overlap reactions) within a cascade as well as cascade-induced de-trapping and/or rotation of defect clusters (beam activated motion). This model accounts for beam activated motions by including a non-thermally activated term in the diffusion coefficient for dislocation loops governed by the average distance between jumps of a loop, that is presumably governed by the interstitial impurity concentration, and frequency of the sudden jumps the visible defects exhibit, which is proportional to the ion flux. Pending a rigorous analysis to determine these quantities precisely, they are currently treated as fitting parameters within the constraints of qualitative observations of the loop motion.

Figure 38 shows the areal density of visible defects predicted by the cluster dynamics model from 0 to $2 \mathrm{dpa}$, as a function of irradiation temperature. $\mathrm{s}$ for two cluster dynamics models. In the thermal model, only the thermally activated diffusion behavior is considered with an activation energy for large, visible interstitial clusters that is consistent with the Arakawa observations, while the beam assisted model includes the beam activated motion effects discussed above. Clearly, a traditional, thermal only diffusion model does not capture the observed experimental trends and predicts breakaway growth of the visible defect cluster density as a result of an unchecked increase in cluster nucleation sites. However, by including a beam assisted diffusion, the model predicts a fast approach to saturation of the defect density, as well as a temperature dependent saturation density that is in good agreement with the experimental observations. This appears to indicate that irradiation induced motions provide the dominant kinetic pathway for the formation of extended defects when dislocation loops are not thermally mobile. Future modeling work will provide a more thorough comparison with the experimental results, in addition to evaluating the effects of one dimensional reaction kinetics for the mobile defect clusters and the development of reaction rates to describe cascade overlap reactions. 




Figure 38. Spatially dependent cluster dynamics model predictions of visible defect cluster density as a function of dose and irradiation temperature for $1 \mathrm{MeV} \mathrm{Kr}$ ion irradiation of thin film, $\mathrm{Fe}-\mathrm{Cr}$ alloy. Two different set of mobility functions have been included for interstitial defect clusters. The thermal only model (dashed lines) allows interstitial clusters to migrate with a diffusivity consistent with the in-situ experimental observations of Arakawa and co-workers, while the beam assisted mobility model superimposes an additional diffusion term that is independent of temperature but has a jump distance and frequency term scaled to experimental considerations.

First principles-based modeling of Ti-Y-O Cluster Energetics in NFAs:

Our general method for the initial stage of this modeling task was to assemble different clusters of $\mathrm{Ti}, \mathrm{Y}$, and $\mathrm{O}$ consisting of up to 20 atoms in $\mathrm{Fe}$ and calculate their energies using electronic structure methods (via the VASP code). In brief, we have assembled a large catalogue of clusters of two different types: on-lattice clusters and structure-matched clusters. The former are clusters that are coherent with the bcc Fe matrix, while the latter are clusters that structurally resemble bulk oxide phases and are consequently not fully coherent with the Fe matrix. We further categorize the structure-matched clusters by their oxygen content, referring to them as hyperstoichiometric, hypostoichiometric, or stoichiometric. The structure-matched clusters are based upon bulk oxide phases with a well-defined stoichiometry, however because they are very small clusters of these bulk phases, their overall composition is dominated by their surface termination. Clusters which are O-terminated are hyperstoichiometric with respect to the bulk oxide phase to which they are matched, Ti- or Y-terminated clusters are hypostoichiometric, and 
clusters that are constrained to be stoichiometric with respect to their parent bulk oxide phase are necessarily of mixed termination.

To calculate the formation energy of a given cluster, we use the following expression:

$$
\Delta E_{f}=E\left(x_{F e}, y_{M}, z_{O}\right)-\left(x \mu_{F e}+y \mu_{M}+z \mu_{O}\right)
$$

where $E\left(x_{F e}, y_{M}, z_{O}\right)$ is the energy of a supercell containing $x$ Fe atoms and a cluster containing $y$ $\mathrm{M}$ atoms (where $\mathrm{M}=\mathrm{Ti}$ or $\mathrm{Y}$ ) and $z \mathrm{O}$ atoms and $\mu_{i}$ is the energy per atom of species $i$ in its reference state. The reference state of $\mathrm{Fe}$ is pure $\mathrm{Fe}$, the reference states for $\mathrm{Ti}$ and $\mathrm{Y}$ are isolated substitutional $\mathrm{Ti}$ or $\mathrm{Y}$ atoms in otherwise pure $\mathrm{Fe}$, and the reference state for $\mathrm{O}$ is an isolated octahedral interstitial $\mathrm{O}$ atom in otherwise pure Fe.

The results of our cluster formation energy calculations for Ti-O and $\mathrm{Y}-\mathrm{O}$ clusters are depicted in Figure 39.
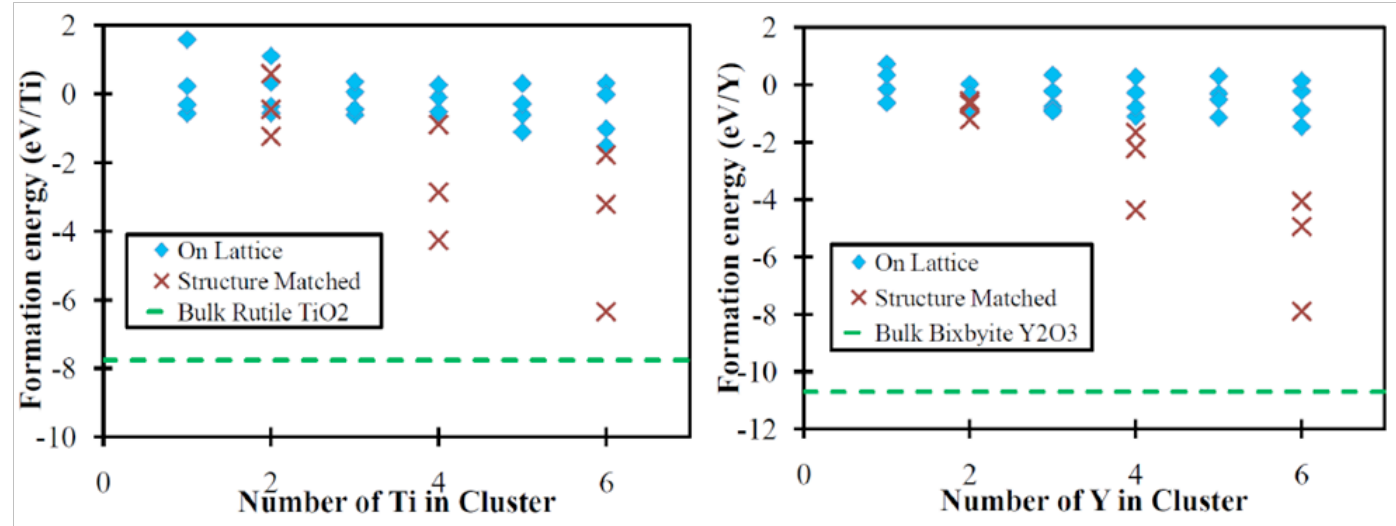

Figure 39. Cluster formation energies as a function of cluster size for Ti-O clusters (left) and YO clusters (right).

For both the Ti-O and Y-O systems, clusters that are similar in structure to bulk Ti or Y oxide phases are far more stable than clusters that match the bcc structure of the Fe lattice. Figure 40 depicts the results of a set of Ti-Y-O clusters with a Ti:Y ratio of 1:1, chosen to match that of the Ti-Y oxide $\mathrm{Y}_{2} \mathrm{Ti}_{2} \mathrm{O}_{7}$ pyrochlore. Cluster energies calculated in similar previous studies are included as well. 


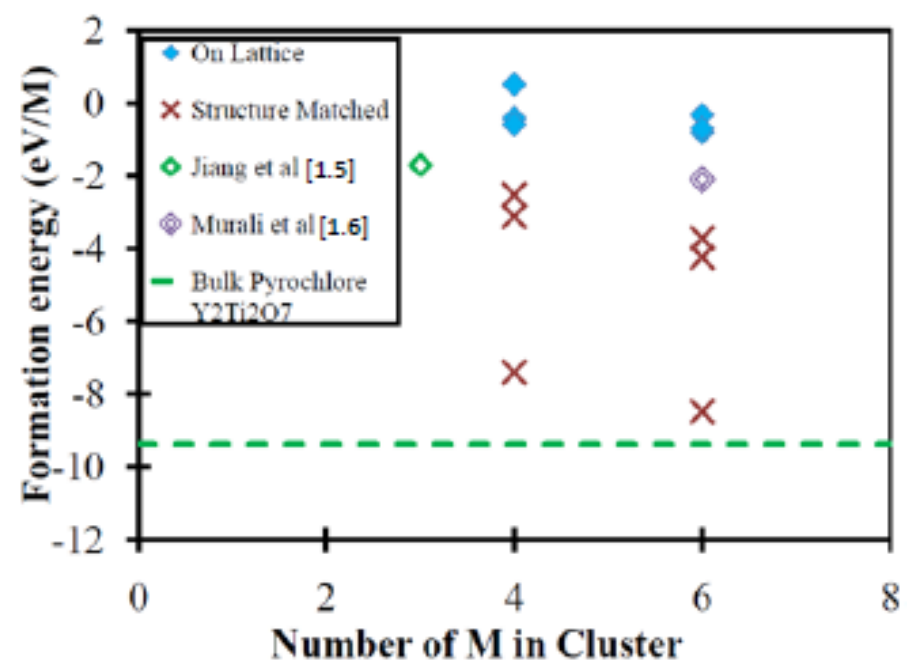

Figure 40. Cluster formation energies as a function of cluster size for Ti-Y-O clusters.

Similar to the Ti-O and Y-O clusters, the structure-matched Ti-Y-O clusters are far more stable than the on-lattice clusters, and are more stable than Ti-Y-O clusters assembled in previous studies as well.

Among the structure-matched clusters, we find that for all cluster sizes, the hyperstoichiometric clusters are most stable, and the hypostoichiometric clusters are least stable. To better understand how this conclusion might depend on the chosen reference state of oxygen, we recalculated the formation energies for the Ti-O and $\mathrm{Y}-\mathrm{O}$ structure matched clusters as a function of oxygen reference chemical potential. We can determine a chemical potential of $\mathrm{O}$ as a function of oxygen partial pressure $\left(\mathrm{PO}_{2}\right)$ and temperature that is referenced to oxygen energies in the DFT calculations. We can then determine M-O cluster formation energy for any reference $\mathrm{PO}_{2}$ of interest. In Figs. 41a and 41b, the formation energies of the structure-matched clusters containing six metal atoms are plotted as functions of reference $\mathrm{PO}_{2}$ at a temperature of $1100 \mathrm{~K}$ for Ti-O and Y-O clusters, respectively. Annotations along the X-axis of Fig. 41a and b denote $\mathrm{PO}_{2}$ values relevant to NFA fabrication. 

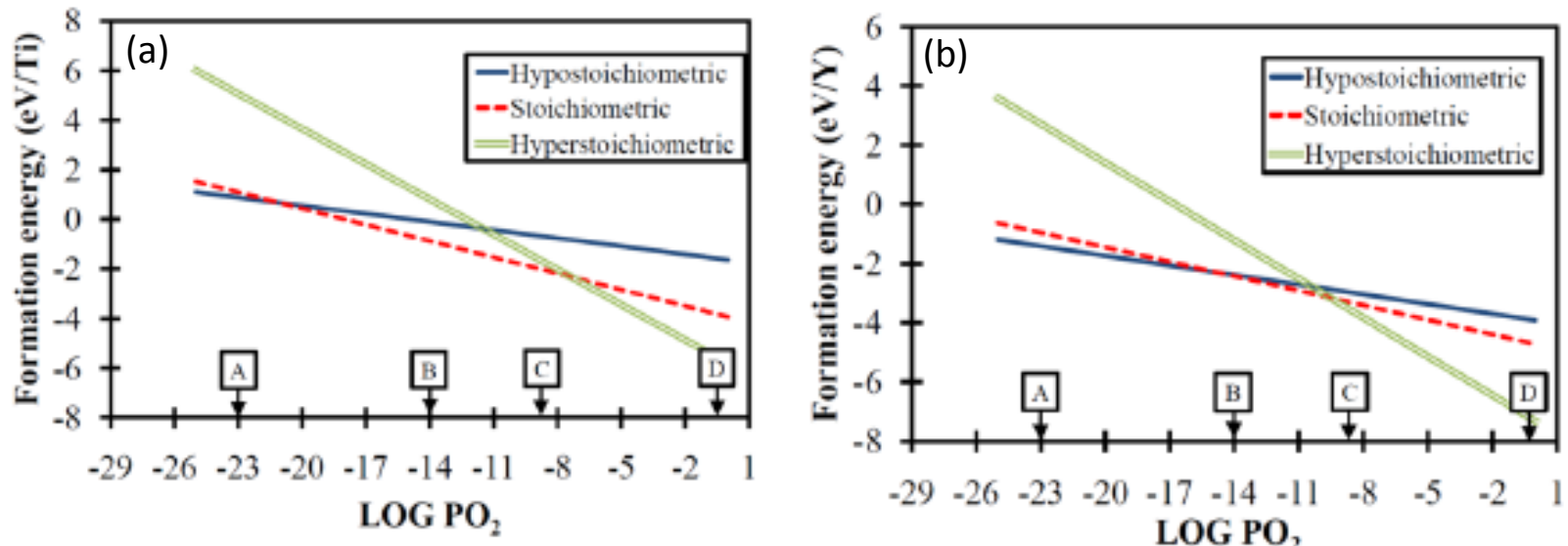

Figure 41 a(left) and b (right): Formation energies of structure-matched Ti-O (left) Y-O (right) clusters containing $6 \mathrm{Y}$ as a function of reference $\mathrm{O}_{2}$ partial pressure. The annotations along the horizontal axis denote the $\mathrm{O}_{2}$ partial pressures corresponding to the following: (a) the partial pressure of $\mathrm{O}_{2}$ at which the reaction $4 \mathrm{Cr}+3 \mathrm{O}_{2} \leftarrow \rightarrow 2 \mathrm{Cr}_{2} \mathrm{O}_{3}$ is in equilibrium. (b) the partial pressure of $\mathrm{O}_{2}$ at which the reaction $2 \mathrm{Fe}+\mathrm{O}_{2} \leftarrow \rightarrow 2 \mathrm{FeO}$ is in equilibrium. C. typical atmospheric partial pressure of $\mathrm{O}_{2}$ during NFA fabrication. D. partial pressure of $\mathrm{O}_{2}$ corresponding to the $\mathrm{O}$ reference state of an isolated $\mathrm{O}$ interstitial in Fe, as calculated via DFT.

From Figure 41, it is evident that the $\mathrm{O}$ reference state used here reflects a high $\mathrm{O}$ chemical potential (denoted by point $\mathrm{D}$ on the $\mathrm{PO}_{2}$ axis in Figs. 41a and b), equivalent to an $\mathrm{O}$ partial pressure of about $5 \mathrm{~atm}$ at $1100 \mathrm{~K}$. As NFAs are typically processed under vacuum, a lower chemical potential may be more appropriate as a reference state when considering nanocluster formation (point $\mathrm{C}$ ). On the other hand, the mechanical alloying process used in NFA fabrication introduces large quantities of $\mathrm{O}$ into the $\mathrm{Fe}$ matrix, and there is experimental evidence that the $\mathrm{O}$ content remains quite high even after the alloy powder is consolidated. This high oxygen content would correspond to a quite high oxygen chemical potential. It is unclear when or indeed if the $\mathrm{O}$ content in the $\mathrm{Fe}$ matrix reaches equilibrium with the atmosphere, and a high $\mathrm{O}$ chemical potential may in fact more relevant to nanocluster formation. Also denoted on Figs. 41 $\mathrm{a}$ and $\mathrm{b}$ are equilibrium $\mathrm{PO}_{2}$ values for selected oxides of $\mathrm{Fe}$ (point $\mathrm{B}$ ) and $\mathrm{Cr}$ (point $\mathrm{A}$ ). Not shown is the equilibrium $\mathrm{PO}_{2}$ for the reactions:

$$
\begin{aligned}
& \mathrm{Ti}+\mathrm{O}_{2} \leftrightarrow \mathrm{TiO}_{2} \\
& 2 \mathrm{Y}+\frac{3}{2} \mathrm{O}_{2} \leftrightarrow \mathrm{Y}_{2} \mathrm{O}_{3}
\end{aligned}
$$

which, according to our calculated energies, occur at approximately $10^{-26}$ and $10^{-65}$ atm at 1100 $\mathrm{K}$, respectively.

Due to the nature of NFA fabrication it is difficult to determine the most appropriate reference state for $\mathrm{O}$ prior to nanocluster formation. However it is useful to note that for $\mathrm{O}$ in equilibrium with $\mathrm{FeO}$ (a $\mathrm{PO}_{2}$ of approximately $10^{-14} \mathrm{~atm}$, from Fig. 41) Ti-O and Y-O nanocluster formation is still energetically favorable. Ti-O and Y-O nanocluster formation should therefore not be thermodynamically inhibited by competition with $\mathrm{FeO}$, even if $\mathrm{FeO}$ is 
already present as a bulk phase and competing for available oxygen (for example as an oxide layer on the surface of the mechanically alloyed powder). As NFAs contain a significant amount of $\mathrm{Cr}$, the formation of $\mathrm{Cr}_{2} \mathrm{O}_{3}$ must also be considered. Contrary to $\mathrm{FeO}$, for $\mathrm{O}$ in equilibrium with $\mathrm{Cr}_{2} \mathrm{O}_{3}$, the Ti-O clusters are energetically unfavorable, while the $\mathrm{Y}-\mathrm{O}$ clusters remain favorable. This analysis suggests that in the presence of $\mathrm{Cr}_{2} \mathrm{O}_{3}$, Ti-O nanocluster formation may be inhibited and that $\mathrm{Y}$ is necessary to nucleate thermodynamically stable clusters.

\section{Kinetic modeling of nucleation and growth processes in NFAs:}

The first stage of this project was devoted to modeling the clustering tendencies and energetics of $\mathrm{Ti}, \mathrm{Y}$, and $\mathrm{O}$ clusters in Fe through the use of density functional theory calculations. The most significant result of this work, discussed above, is that Ti-Y-O clusters that structurally resemble bulk metal oxides are substantially more stable than clusters that strictly maintain the bcc structure of the Fe lattice. Coupled with recent high resolution TEM results which find that the smallest precipitates in a Ti-Y-O NFA are crystallites of the complex oxide $\mathrm{Y}_{2} \mathrm{Ti}_{2} \mathrm{O}_{7}$, this result provides theoretical basis for treating the evolution of the Ti-Y-O nanoprecipitates in NFAs as the nucleation and growth of the known oxides of Ti and $\mathrm{Y}$ in Fe.

Therefore, based upon the above conclusions, the effort toward simulating precipitate evolution in NFAs was devoted to the development of a rate theory model of nucleation and growth based on the theories of Slezov and Schmelzer. This approach is based upon solving the following master equation for the time evolution of the cluster size distribution:

$$
\frac{\partial f(n, t)}{\partial t}=w_{n-1, n}^{(+)} f(n-1, t)-w_{n, n-1}^{(-)} f(n, t)+w_{n+1, n}^{(-)} f(n+1, t)-w_{n, n+1}^{(+)} f(n, t)
$$

where $f(n, t)$ is the concentration of clusters containing $n$ atoms at time $t$. The coefficients $w_{i, j}^{(+)}$ are the rates at which clusters of size $i$ aggregate single atoms to grow to size $j$. Similarly, the coefficients $w_{j, i}^{(-)}$are the rates at which clusters of size $j$ emit single particles to shrink to size $i$.

For the rates of aggregation, we adopt the simplified form provided by Bellon and Martin:

$$
w_{n-1, n}^{(+)}=\frac{4 \pi R_{n-1} D}{\Omega} f(1, t)
$$

where $R_{n}$ is the radius of a particle of size $n, D$ is the diffusion coefficient of monomers in the ambient phase, and $\Omega$ is the volume per atom in the precipitating phase. $f(1, t)$ represents the concentration of clusters of size 1 , or, equivalently, the concentration of monomers in the ambient phase.

Once the coefficients of aggregation have been determined, the coefficients of emission may be calculated based upon the following expression:

$$
\frac{w_{n-1, n}^{(+)}}{w_{n, n-1}^{(-)}}=\exp \left(-\frac{[\Delta G(n)-\Delta G(n-1)]}{k_{B} T}\right)
$$


where $\Delta G(n)$ is the change in the total free energy corresponding to the formation of a cluster of size $n$ from $n$ monomers in the ambient phase. Combining (6) with (7) and (8) yields

$$
\begin{aligned}
& \frac{\partial f(n, t)}{\partial t}=w_{n-1, n}^{(+)}\left\{f(n-1, t)-f(n, t) \exp \left[\frac{\Delta G(n)-\Delta G(n-1)}{k_{B} T}\right]\right\}+ \\
& w_{n, n+1}^{(+)}\left\{-f(n, t)+f(n+1, t) \exp \left[\frac{\Delta G(n+1)-\Delta G(n)}{k_{B} T}\right]\right\}
\end{aligned}
$$

For a cluster size distribution containing cluster size classes from 1 to $n$, the partial differential equation of eqn. (9) may be solved by discretizing into $n$ coupled ordinary differential equations, one for each cluster size class, and integrating numerically. From the resulting size distribution solution, relevant parameters such as mean particle radius and cluster density may be easily derived.

The model description thus far has been limited to the precipitation of a single component phase. However, it may be used to treat the precipitation of stoichiometric phases of constant composition. In such a description, $n$ is the number of formula units in a given cluster, rather than the number of discrete atoms. As a validation of the model, we have used it to simulate the precipitation and growth of $\mathrm{Y}_{2} \mathrm{O}_{3}$ in $\mathrm{Fe}$, based upon the physical parameters used in the classical nucleation and growth model of Hin et al.

\section{Validation of modeling approach}

Figure 42 depicts the mean $\mathrm{Y}_{2} \mathrm{O}_{3}$ particle size (left) and the cluster density (right) in an $\mathrm{Fe}-15 \mathrm{Cr}$ $0.13 \mathrm{Y}-0.18 \mathrm{O}$ alloy, as predicted by the classical nucleation and growth model of Hin et al, as predicted by the precipitation model described above using the physical parameters of Hin et al, and as measured experimentally.
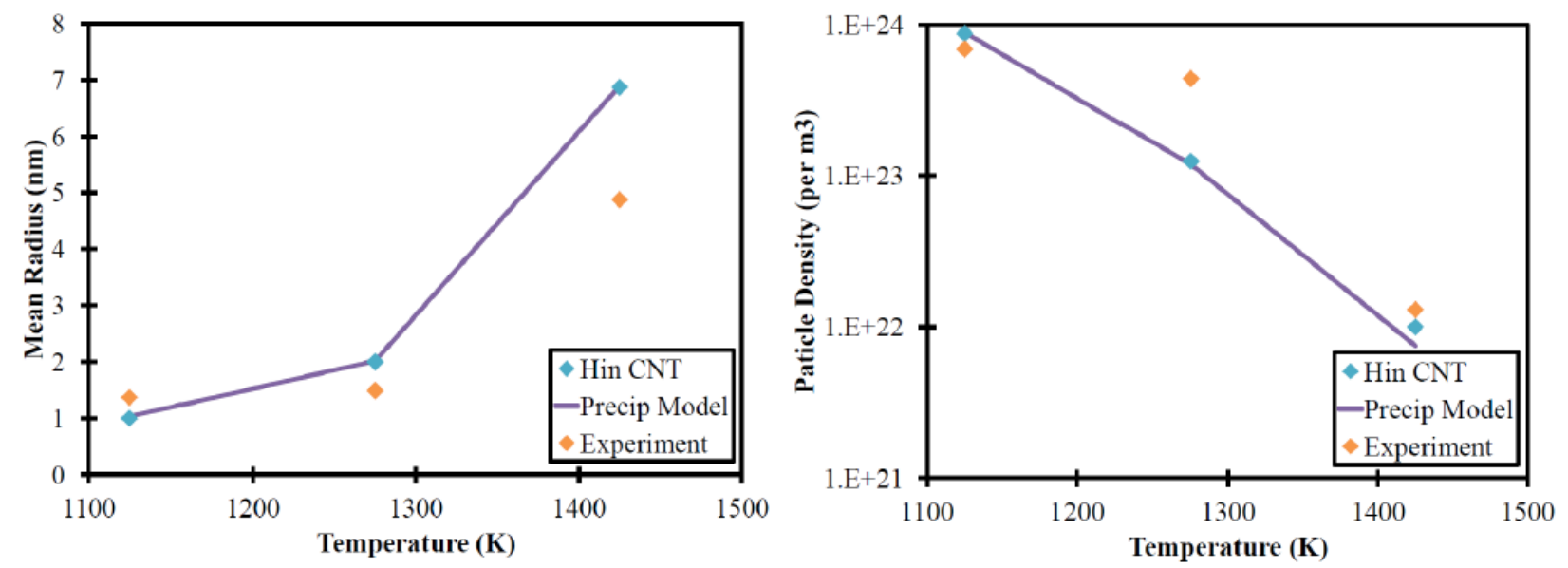

Figure 42. Mean precipitate size (left) and precipitate density (right) values for $\mathrm{Y}_{2} \mathrm{O}_{3}$ precipitation in $\mathrm{Fe}$, from theory and experiment. 
The parameters used in the work of Hin et al are derived from a best fit to the experimental data set depicted in Figure 42. The excellent agreement our data with the model results from Hin et al demonstrates that we have implemented the model correctly.

Radiation-induced precipitation:

The potential for delayed formation of very high volume fractions (up to several \%) of $\mathrm{Mn}-\mathrm{Ni}-\mathrm{Si}$ nano-scale precipitates (MNSPs) in LWR reactor pressure vessel (RPV) steels is arguably one of the most immediately important safety issue in the entire field of nuclear reactor materials, since these precipitates will cause the embrittlement of the RPV steels, which may limit plant life extension (PLE) of operating nuclear reactors. Here we've developed a thermodynamic as well as a kinetic model to study the formation of these precipitates in typical RPV steels and compared to experimental data from Professor Odette's group at University of California - Santa Barbara. The work consists three parts: First, the equilibrium state of typical RPV steels were studied with two different thermodynamic databases, one is simplified "in-house" database while the other is commercial TCAL2 database, and results were compared to experimental data at high fluence in ATR1. The comparison of results between the two databases gave a sense of the accuracy of simplified "in-house" database while comparison between modeling results and experimental data would indicate if any additional irradiation effect should be considered in thermodynamic models. Also, these thermodynamic studies can provide parameters to kinetic studies of precipitates and change of mechanical properties of materials. Second, the solubility of Fe in precipitates was studied with ab initio method as coded in VASP, which uses density functional theory to describe atomic interactions. This study would address the issue if Fe exits in the precipitates, since some APT experiments have observed the existence of Fe in precipitates but this may also be just artifacts due to the small size of these precipitates. This would support our following study of the kinetics of these precipitates. Third and finally, the evolution of these $\mathrm{Mn}-\mathrm{Ni}$-Si rich precipitates was studied with cluster dynamics method with thermodynamic parameters both from "in-house" database and TCAL2 database and the results were compared to experimental data. This work allows the prediction of long-term evolution of these precipitates under real reactor conditions, although the results are not yet accurate enough for robust prediction.

There are 12 ternary compounds in the Mn-Ni-Si system, which are all included in the present "in-house" database as the potential precipitation in the bcc matrix in this study. The CALPHAD-type thermodynamic parameters of these compounds are directly taken from the thermodynamic description performed by $\mathrm{Hu}$ et al. According to the work by $\mathrm{Hu}$ et al., there are 7 ternary compounds in the isothermal section at $550 \mathrm{~K}$. As shown in Figure 43, the ternary compounds, T3, T7, T9 and T10, are considered as the stoichiometric phase, while T6 and T8 are the two non-stoichiometric ternary compounds. It can be easily observed that, in Fig. 43, the compositions of precipitates are well predicted using both "in-house" and TCAL2 databases. The black solid symbols are the composition of precipitates determined by the work of Wells et al., while the blue ones are the prediction from the "in-house" database, and the red open ones from TCAL2 databases. 


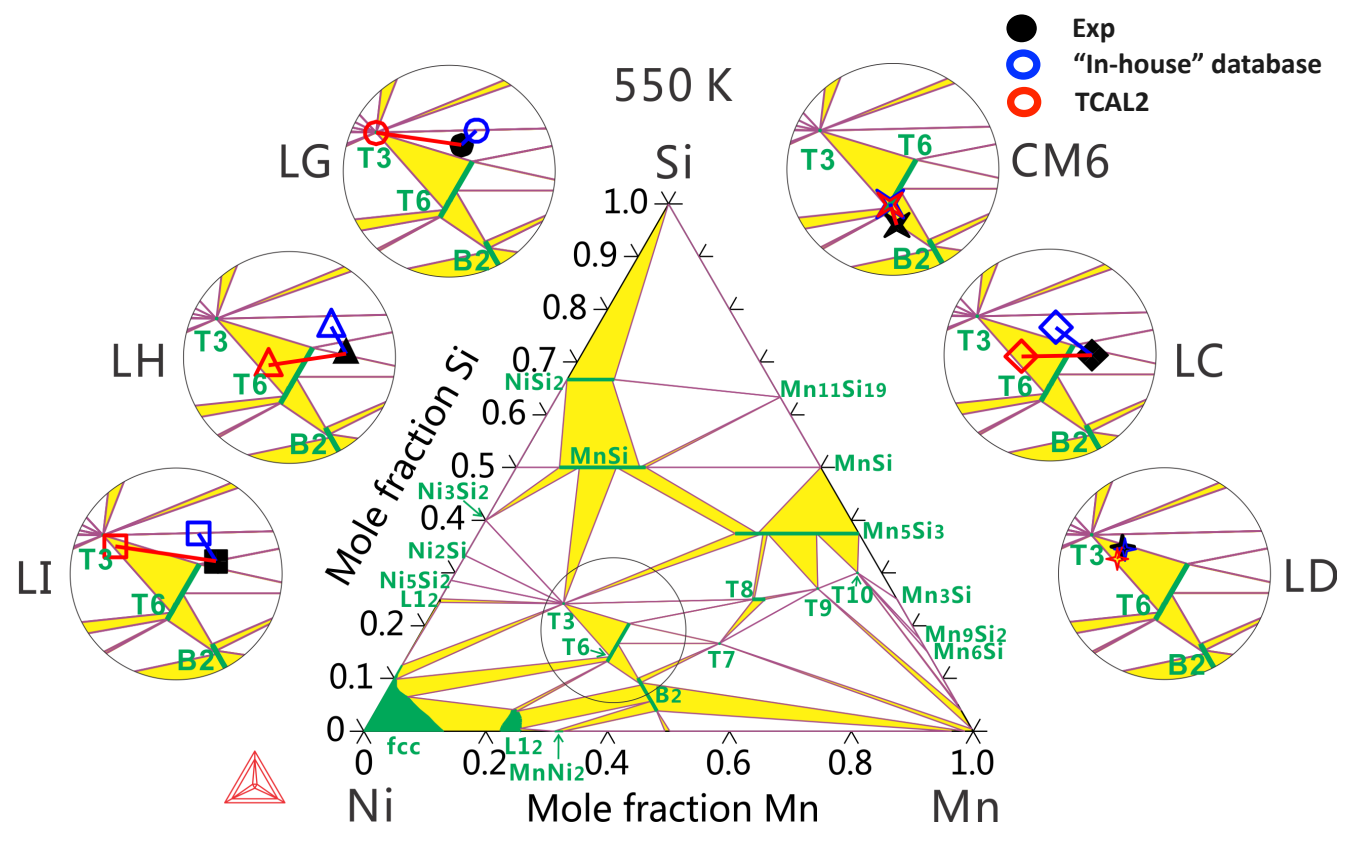

Figure 43. Isothermal section of the Mn-Ni-Si system at 550K. Red symbols are the calculated compositions of precipitates from the commercial Thermo-Calc database (TCAL2), while the ones in Blue are from the in-house database.

Figure 44 is the quantitative comparison of composition of each element between the CALPHAD databases and experimental measurements. It is found that the "in-house" database shows better agreement of Mn and Ni than the TCAL2 database, while TCAL2 obtains slightly better prediction of the Si content in precipitates. 

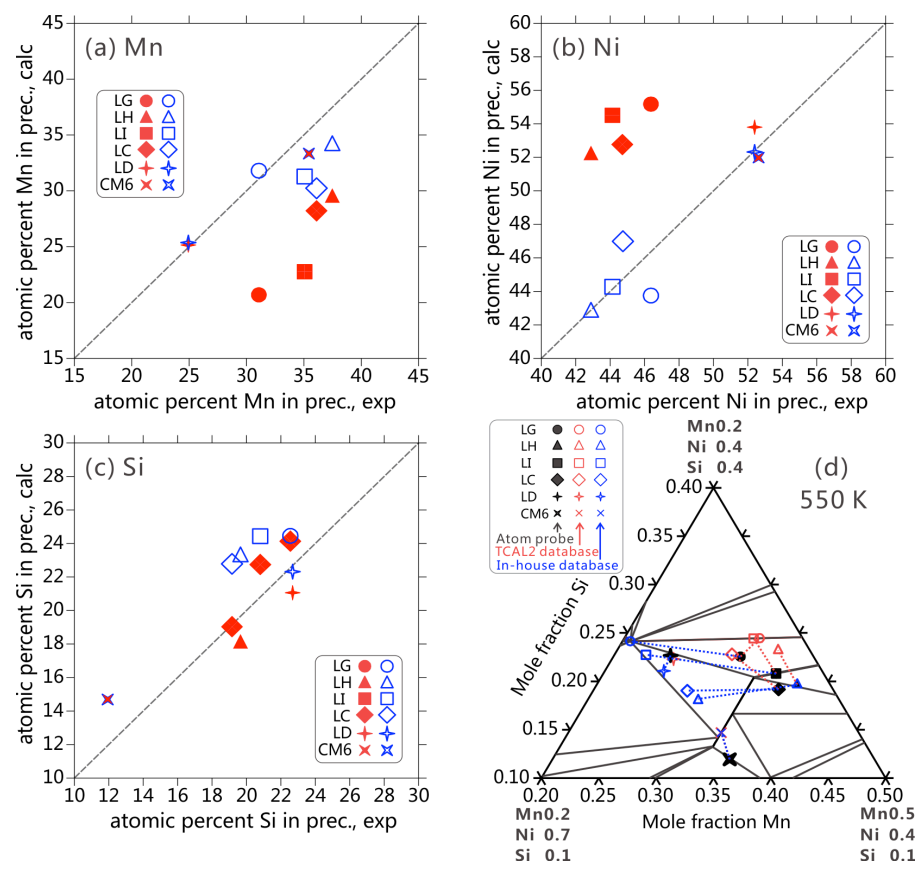

Figure 44. Comparison of compositions of precipitates predicted by in-house and commercial databases. The blue open symbols are the calculated results using the "in-house" database, while the red solid ones are from commercial database.

The solute percentage, which is defined as the fraction of solute atoms in the system that come out to precipitates, is one of the most important factors, which determines the limit of the precipitates in the coarsening stage. According to Figure 45, both the current simplified model and the one in the TCAL2 database can predict reasonable value of the solute percentage in the alloys, although a significant deviation can be observed for $\mathrm{Si}$ as shown in Fig. 45c. This $\mathrm{Si}$ result is due to the larger Si activity in the bcc matrix found in the "in-house" database than the TCAL2 database and which activity is correct is under investigation.

Figure 46 shows the fraction of each phase at equilibrium state that we predicted with both "inhouse" database and TCAL2 in different alloys. And Table 4 gives the available structure information for these phases. According to Fig. 46, the T8 compound will appear in the LG, LH, LI and LC alloys using the "in-house" database. However, the calculation based on the TCAL2 database does not allow the T8 phase precipitating in the bec matrix. Although it would be natural to believe that the commercial database with more phases involved in the thermodynamic description would have a better quality in the prediction, the plot for LG shown in Fig. 46 indicates that T6 and T8 phases could be the precipitates in this alloy, which is not predicted by the TCAL2 database. In fact, due to the complexity of the isothermal section of the $\mathrm{Mn}-\mathrm{Ni}$-Si system at $550 \mathrm{~K}$, the prediction of the phases in precipitates is highly sensitive, and thus quite hard to match the exact phase field found in the atom probe results shown in Figure 43. But this sensitivity does not mean that the thermodynamic database would fail the prediction, on the contrary, the good consistency of the comparison shown in Figure 44 and Figure 45 indicates that the present thermodynamic models could provide valuable information for further experimental investigation on these RPV alloys. 



Figure 45. Comparison of solute fractions of precipitates predicted by in-house and commercial databases. The blue open symbols are the calculated results using the in-house database, while the red solid ones are commercial database.

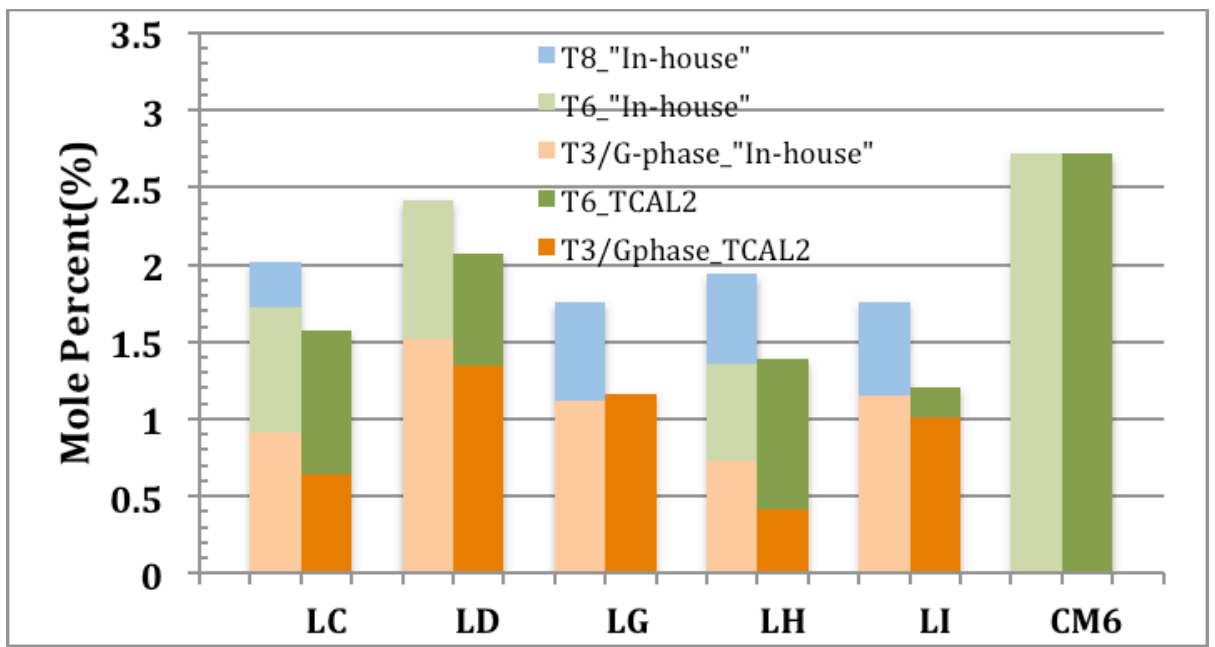

Figure 46. Predicted mole fraction of phases in precipitates at $550 \mathrm{~K}$. 
Table 4. Structure information for possible Mn-Ni-Si precipitation in RPV steels.

\begin{tabular}{|c|c|c|c|}
\hline Phase & Composition & Space group & Lattice Parameter $(\AA)$ \\
\hline T3 & $\mathrm{Mn}_{6} \mathrm{Ni}_{16} \mathrm{Si}_{7}$ & Fm3m & 11.4 \\
\hline $\mathrm{T} 6$ & $\mathrm{Mn}(\mathrm{Ni}, \mathrm{Si})_{2}$ & $\mathrm{Fd} 4 \mathrm{~m}$ & 6.67 \\
\hline $\mathrm{T} 8$ & $(\mathrm{Mn}, \mathrm{Ni})_{3} \mathrm{Si}$ & - & - \\
\hline
\end{tabular}
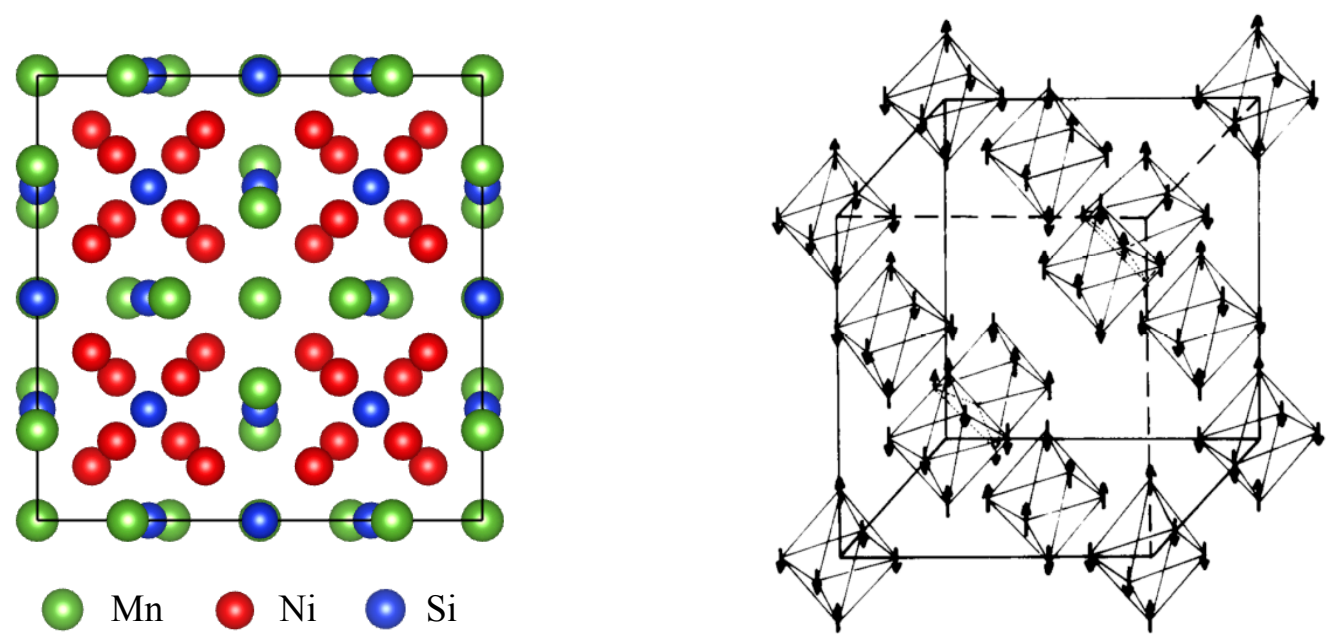

Figure 47. Crystal and magnetic structure of G-phase.

One issue with the above models is that it is assumed that the G-phase does not contain any Fe. According to previous thermodynamic calculations, T3/G-phase is predicted in five of the six alloys and in a large proportion. According to some Atom Probe experiments, there is some $\mathrm{Fe}$ in the precipitates, but this maybe just an artifact due to the small size of these precipitates. Here will use $a b$ initio method as coded in the VASP to study the solubility of Fe in G-phase.


Figure 48. Fe Solubility in G-phase a) By replacing Mn b) By replacing Ni 
G-phase is a stoichiometric phase with composition $\mathrm{Mn}_{6} \mathrm{Ni}_{16} \mathrm{Si}_{7}$ in space group Fm3m. The lattice constant is about $1.14 \mathrm{~nm}$, which is about 4 times that of bcc Fe. Figure 47 shows the crystal and magnetic structure of G-phase with 116 atoms in the unit cell.

Based on our $a b$ initio calculation results, Figure 48 shows the solubility of Fe in G-phase at different temperatures, from which we can see that there is almost no Fe solubility (less than $0.05 \%$ when replacing $\mathrm{Mn}$ and $0.005 \%$ when replacing $\mathrm{Ni}$ ) under typical RPV steel conditions.

We first examined the precipitation behavior of $\mathrm{Fe}-\mathrm{Cu}$ model alloy as a test of our model. We consider both ageing and irradiated precipitation.

Ageing
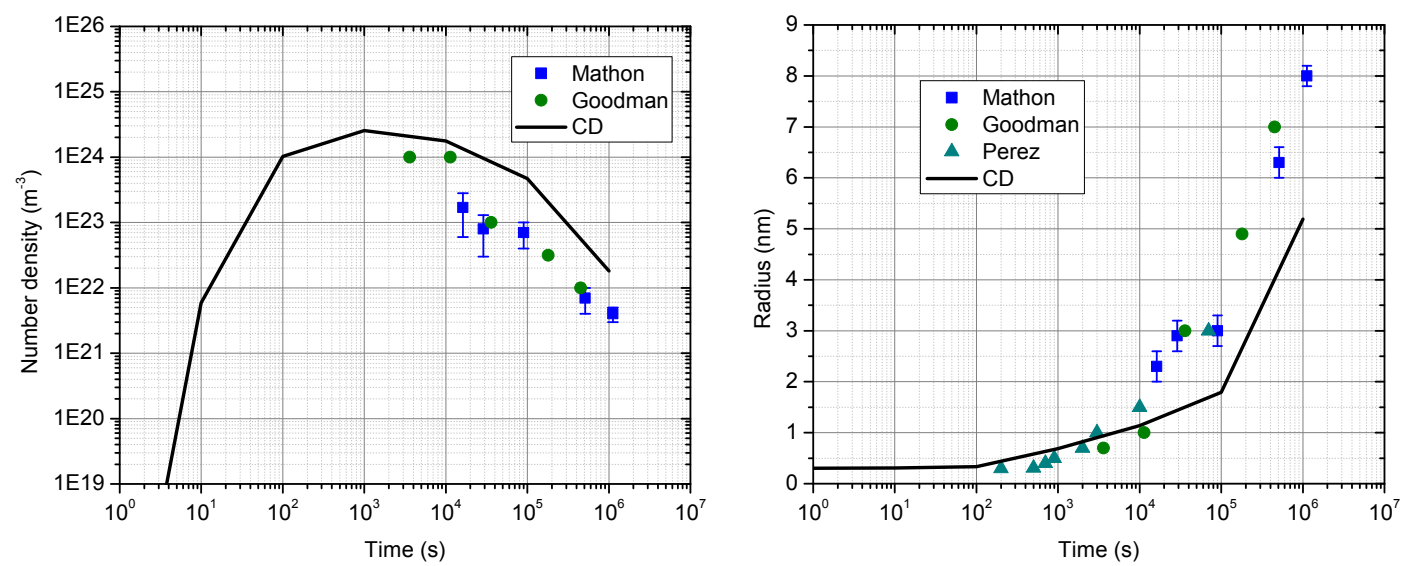

Figure 49. Results of cluster dynamics simulation compared to experimental data (left) Number Density (right) Mean Radius.

The diffusion coefficient of $\mathrm{Cu}$ in bcc Fe is from experiment. Figure 49 show the comparisons of our simulation results compared to corresponding experimental data, which were all performed at $500^{\circ} \mathrm{C}$. Since these compositions are very close to each other they have little effect on the number density and mean radius of the precipitation and we put them together for comparison here. The simulation is done with composition $\mathrm{Fe}-1.34 \mathrm{at} . \% \mathrm{Cu}$. From these figures we can see that simulation results are in good agreements with experimental data. 

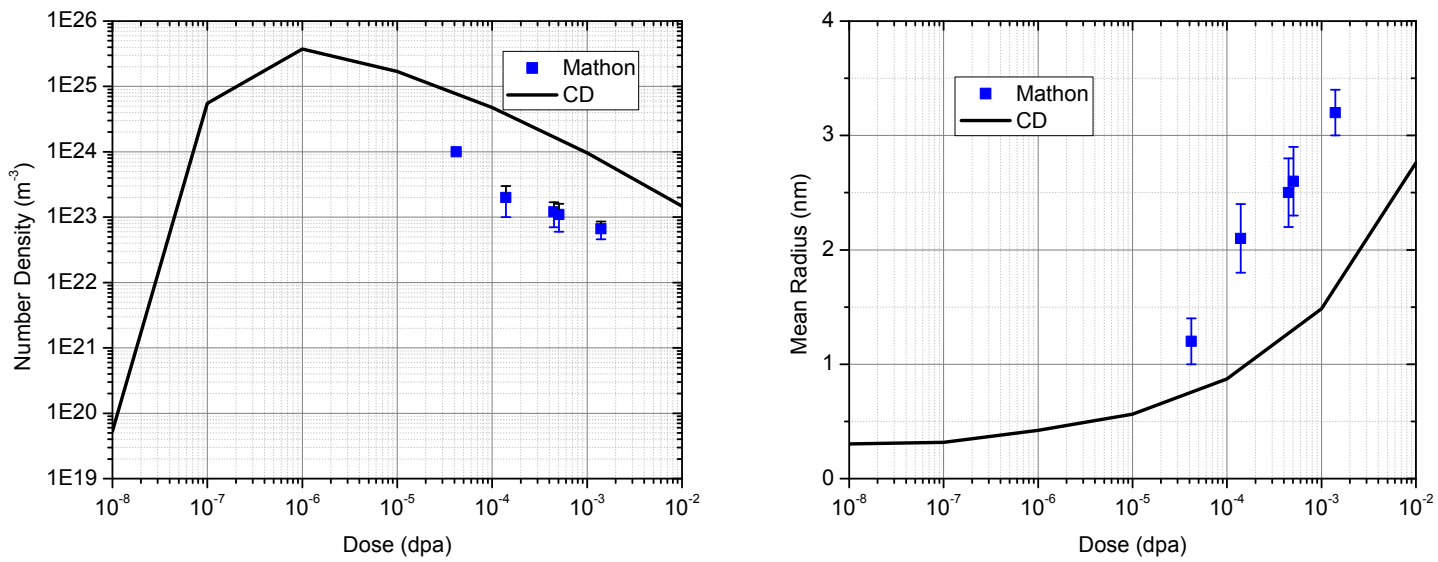

Figure 50. CD simulation results compared to experimental data under irradiation (left) Number Density (right) Mean Radius.

Figure 50 shows the simulation results of $\mathrm{Fe}-1.34 \mathrm{at} . \% \mathrm{Cu}$ under irradiation at a dose rate of $1 \times 10^{-8} \mathrm{dpa} / \mathrm{s}$ at $300^{\circ} \mathrm{C}$. Parameters for the irradiation enhanced diffusion are obtained from Faulkner's paper. Results are compared with experimental data done by Mathon. The result is qualitatively consistent with experimental data, but discrepancy between simulation and experiment still exists.


Figure 51. Cluster Dynamic results with in-house database compared with experimental data a) Number Density b) Mean Radius.

Since the possible $\mathrm{Mn}-\mathrm{Ni}$-Si rich precipitates are close in energetics and G-phase is predicted in five of the six studied alloys, we studied the kinetics of these precipitates by assuming the precipitation is stoichiometric G-phase and compared with experimental data with interfacial energy as a fitting parameter. Both TCAL2 and our "in-house" database were used as thermodynamic input parameter, respectively. Figure 51 shows the comparison between cluster dynamic result with "in-house" database with experimental data for number density and mean radius. Here the fitting interfacial energy we obtained is $650 \mathrm{~mJ} / \mathrm{m}^{2}$, which is a little bit higher 
compared to the interfacial energy of $\mathrm{Cu}$ precipitation in $\mathrm{Fe}\left(400 \mathrm{~mJ} / \mathrm{m}^{2}\right)$, but is still in the reasonable range.

Figure 52 shows the comparison between experimental data with cluster dynamic results with TCAL2 database, where the fitting interfacial energy is $85 \mathrm{~mJ} / \mathrm{m}^{2}$. Both these two database give qualitative agreements with experimental data, although some significant differences exist.

The interfacial energy for the TCAL2 database is very low, and probably unphysical. The large difference for the fitting results of interfacial energy between TCAL2 database and "in-house" database is because of the difference of Si activity between these two databases. Si has a much lower solute limit in "in-house" database than that in TCAL2 database.
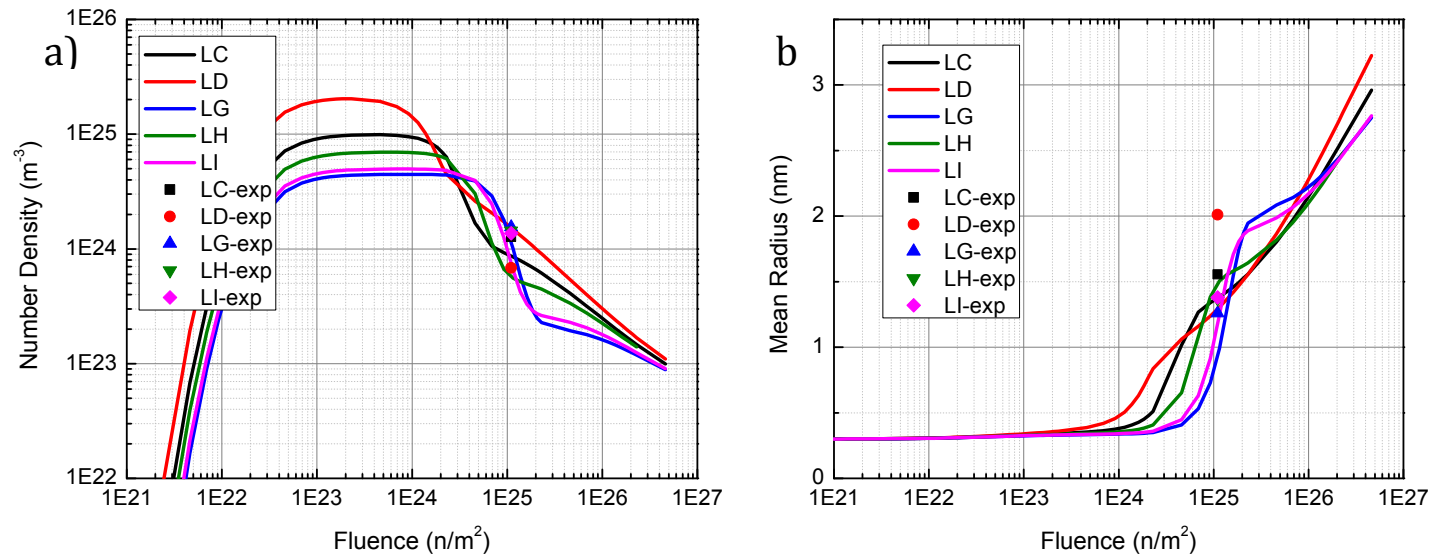

Figure 52. Cluster Dynamic results with TCAL2 database compared with experimental data a) Number Density b) Mean radius.

Figure 53 shows the comparison of volume fraction between cluster dynamic results and experimental data, both with "In-house" database and TCAL2 database. If the dots fall on to the diagonal line, it means the calculation results are exactly the same as the experimental data. From the figure, we can see that both databases give reasonable agreement with experimental data, while TCAL2 gives lower volume fraction compared to experimental data. 

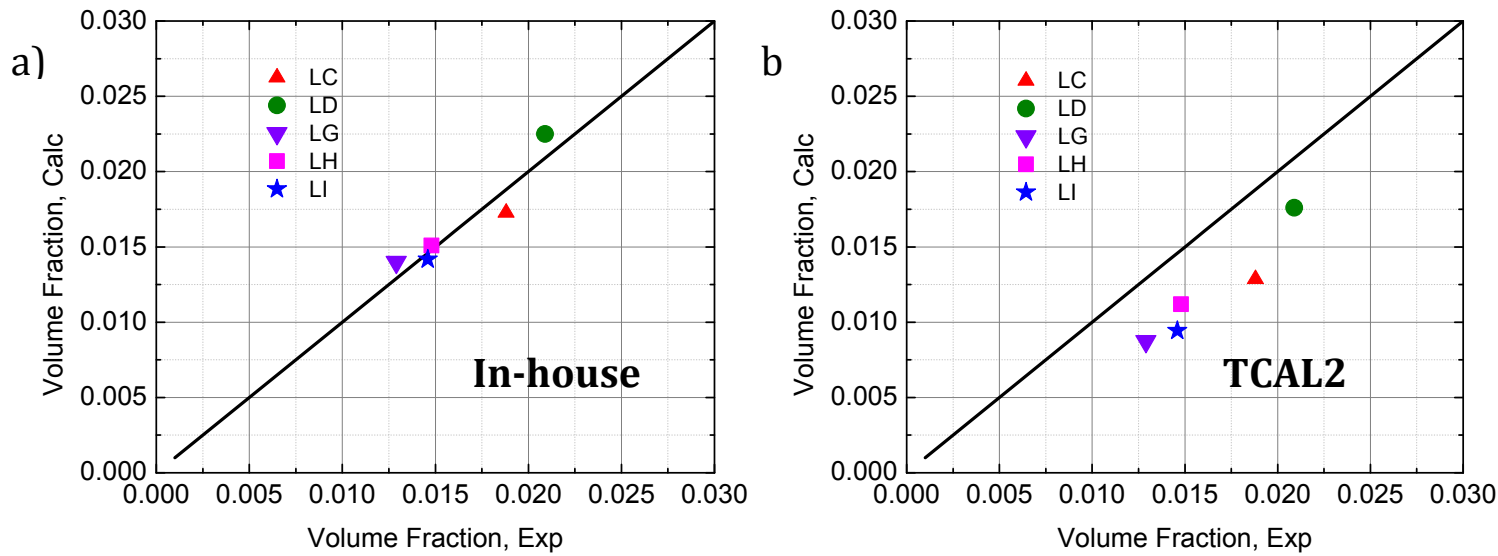

Figure 53. Comparison of volume fraction between calculation results and experimental data a) "In-house" database b) TCAL2 database.

It is encouraging that we find that even with the simplified "in-house" database, the modelprediction shows good consistency with both atom probe experiments as well as, in most cases, the TCAL2 commercial database. More importantly, by comparing with the current "in-house" database and atom probe experimental results, we support the assumption that the precipitation observed in these alloys under irradiation is predominantly governed by thermodynamics, and irradiation serves only to enhance diffusion. Such a finding reveals that the coarse-grained simulations, e.g. cluster dynamics, can directly take their driving forces from the thermodynamic equilibrium CALPHAD models. Furthermore, it should be noticed that, depending on how close accelerated tests are to real extended reactor conditions, these simple thermodynamic models can predict volume fraction of precipitates of arbitrary alloys under life extension. Such a success would provide valuable guidance for studying mechanical properties of RPV steels and mechanism of irradiation embrittlement.

By assuming G-phase as the only kind of precipitate, good qualitative agreements can be obtained between experimental data and simulation results, although even the small difference between thermodynamic models will be reflected in this kinetic study. Overall it is encouraging that cluster dynamics method can give us a quick and qualitative guidance of the long-term growth and coarsening process of the precipitates in RPV steels. 